\title{
Fermion Masses and Mixing in Extended Technicolor Models
}

\author{
Thomas Appelquist ${ }^{a}{ }^{*}$, Maurizio Piai ${ }^{a}{ }^{\dagger}$, Robert Shrock $^{b} \ddagger$ \\ (a) Physics Department, Sloane Laboratory \\ Yale University \\ New Haven, CT 06520 \\ (b) C. N. Yang Institute for Theoretical Physics \\ State University of New York \\ Stony Brook, N. Y. 11794
}

\begin{abstract}
We study fermion masses and mixing angles, including the generation of a seesaw mechanism for the neutrinos, in extended technicolor (ETC) theories. We formulate an approach to these problems that relies on assigning righthanded $Q=-1 / 3$ quarks and charged leptons to ETC representations that are conjugates of those of the corresponding left-handed fermions. This leads to a natural suppression of these masses relative to the $Q=2 / 3$ quarks, as well as the generation of quark mixing angles, both long-standing challenges for ETC theories. Standard-model-singlet neutrinos are assigned to ETC representations that provide a similar suppression of neutrino Dirac masses, as well as the possibility of a realistic seesaw mechanism with no mass scale above the highest ETC scale of roughly $10^{3} \mathrm{TeV}$. A simple model based on the ETC group SU(5) is constructed and analyzed. This model leads to non-trivial, but not realistic mixing angles in the quark and lepton sectors. It can also produce sufficiently light neutrinos, although not simultaneously with a realistic quark spectrum. We discuss several aspects of the phenomenology of this class of models.
\end{abstract}

14.60.PQ, 12.60.Nz, 14.60.St

*email: thomas.appelquist@yale.edu

$\dagger^{\dagger}$ email: maurizio.piai@yale.edu

‡email: robert.shrock@sunysb.edu 


\section{INTRODUCTION}

The hypothesis that electroweak symmetry breaking (EWSB) is triggered by a Higgs potential in which the quadratic term $\mu^{2} \phi^{\dagger} \phi$ has a coefficient $\mu^{2}<0$, leads to the wellknown hierarchy problem, the radiative instability of Higgs sector with respect to a much higher fundamental scale. Various approaches to this problem, such as the incorporation of supersymmetry, have been suggested in which the Higgs field remains elementary to energies well beyond the electroweak scale. However, in two cases where scalar fields have been used successfully to describe spontaneous symmetry breaking in the real world, namely the Ginzburg-Landau free energy functional for superconductivity and the $\sigma$ model for spontaneous chiral symmetry breaking in hadronic physics, the scalars are clearly composite at the relevant energies, representing bilinear fermion condensates.

These facts have motivated an alternative approach based on dynamical EWSB driven by a strongly coupled gauge interaction, associated with an exact gauge symmetry, denoted generically as technicolor (TC) [1]- [5]. The EWSB is generated by the condensation of technifermion bilinears, and the masses of quarks and charged leptons then arise via extended technicolor (ETC) interactions [2]. It has seemed possible to understand some of the fermion mass scales in this way, although the very light neutrino masses present a newer and perhaps more challenging problem. Two of us have shown, however, how light neutrino masses and lepton mixing can be obtained in extended technicolor models [6,8] containing a set of standard-model (SM) singlet neutrinos. This involves a strong suppression of both Dirac

and Majorana neutrino mass terms, along with a seesaw mechanism that does not involve any superheavy mass scales.

In this paper, we explore in a similar way the generation of all the fermion masses and mixing angles. This is a very ambitious task. Grand unified theories are less ambitious in this respect, since they do not incorporate a dynamical theory of generations, but instead put these in as copies of the GUT group representations. A variety of free parameters is available to accommodate measured masses and mixing angles. Extended technicolor theories gauge the generations and must dynamically produce all the fermion masses, the generational hierarchy, the fermion mixing angles, etc. Our work on this problem has met with only partial success. Nevertheless, it seems worthwhile to report our results and discuss open problems.

We begin by reviewing the problem of fermion mass and mixing angle generation in ETC models. The intra-generational mass splittings and CKM mixing have been longstanding challenges for these theories. In this paper we propose a mechanism that succeeds in generating both. We explore this mechanism in an explicit ETC model based on the ETC 
gauge group $\mathrm{SU}(5)$. It incorporates a generational $\mathrm{U}(3)$, and as the $\mathrm{SU}(5)$ breaks sequentially, it leads naturally to a generational hierarchy and a remaining, unbroken $S U(2)_{T C}$ group. A key feature will be the assignment of right-handed $Q=-1 / 3$ quarks and charged leptons to ETC representations $\mathcal{R}$ that are conjugates of those of the corresponding left-handed fermions. This leads to a natural strong suppression of these masses relative to the $Q=2 / 3$ quark masses.

The representations of the SM singlet neutrinos are such that at all but the highest ETC symmetry breaking scales, the left- and right-handed components also transform according to mutually conjugate representations. Thus, the elements of the Dirac mass matrices for neutrinos are also naturally suppressed [3,22]. This suppression, together with the dynamical generation of Majorana mass terms for other SM-singlet neutrinos can provide a new kind of seesaw mechanism $[6,8]$ that involves only mass scales well within the ETC range.

In Section II, we briefly review the technicolor and extended technicolor framework, focusing on the problem of fermion masses and mixing, including the generation of the quark and charged lepton mass hierarchies and mixing angles as well as the typical presence of new, heavy degrees of freedom. The corresponding discussion of neutrino masses and mixing angles is postponed to section IV.

The models of Refs. $[22,6,8]$ are the immediate antecedents of the model to be presented in this paper. They share in common the feature of having two asymptotically-free gauge interactions that become strong at high scales: $\mathrm{SU}(5)_{E T C} \times \mathrm{SU}(2)_{H C}$, where the latter is a new interaction designated as hypercolor (HC). Ref. [6] presented a mechanism for obtaining realistically light neutrino masses and lepton mixing. Ref. [8] showed how this could be implemented in theories with extended strong-electroweak groups. We review these models briefly in Section III.

Section IV is the core of the paper. We begin by describing the ingredients of our model, including the use of relatively conjugate ETC representations. We discuss generally the structure of the neutrino mass matrices and the possible appearance of a seesaw mechanism. Two possible symmetry breaking sequences are then described, each leading to the unbroken $S U(2)_{T C}$ group. In each case, suppressed neutrino masses emerge naturally, as do quark and charged lepton mass splittings and mixing angles. The general structure of the mass matrices is elucidated by a set of selection rules that follow from residual global generational symmetries. For both sequences, however, the success is only partial, with mixings that are not fully realistic and an inability to achieve simultaneously the right level of suppression for both Dirac neutrino masses and down-type quark and charged lepton masses.

In Section V we discuss some phenomenological aspects of this class of models. These include the constraints from precision electroweak data, flavor-changing neutral current pro- 
cesses, and ETC-instanton-induced violation of lepton number. We also discuss global symmetries and associated Nambu-Goldstone bosons. An interesting generic prediction of models that incorporate the mechanism proposed in Ref. [6] for the origin of light neutrino masses, and in particular of the class of models discussed here, is the existence of neutrino-like mass eigenstates that are predominantly electroweak-singlets with masses that lie in the range from a few hundred $\mathrm{MeV}$ to a few hundred $\mathrm{GeV}$. We comment on experimental implications of these particles. We also remark on possible candidates for dark matter.

In Section VI, we summarize our work, listing the successes and shortcomings of the mechanism employed. We suggest possible directions for future study.

\section{GENERAL FRAMEWORK}

\section{A. Technicolor and Extended Technicolor}

For general discussion, we take the technicolor gauge group to be $\mathrm{SU}\left(N_{T C}\right)$. The set of

technifermions includes one family, viz., $Q_{L}=\left(\begin{array}{c}U \\ D\end{array}\right)_{L}, L_{L}=\left(\begin{array}{c}N \\ E\end{array}\right)_{L}, U_{R}, D_{R}, N_{R}, E_{R}$, with each field transforming according to the fundamental or conjugate fundamental representation of $\mathrm{SU}\left(N_{T C}\right)$ and the usual representations of $G_{S M}=\mathrm{SU}(3) \times \mathrm{SU}(2)_{L} \times \mathrm{U}(1)_{Y}$. The $N_{R}$ are SM singlets.

To generate the quark and lepton masses, this theory must be imbedded in a larger, extended technicolor (ETC) theory, taken here to be $\mathrm{SU}\left(N_{E T C}\right)$. Constraints from flavorchanging neutral-current processes require that the ETC vector bosons, which can mediate generation-changing transitions, must have large masses. We envision that they arise from self-breaking of the ETC gauge symmetry, which can occur if ETC is a strongly coupled, chiral gauge theory.

Each fermion with standard-model interactions is embedded in a fundamental or conjugate fundamental representation of the ETC gauge group such that the first three components are the successive generations of this fermion, and the remaining components are the corresponding technifermions with the same SM quantum numbers. This entails the relation

$$
N_{E T C}=N_{g e n .}+N_{T C}=3+N_{T C} .
$$

Additional standard-model-singlet fermions, some of which will play the role of right-handed neutrinos, will also be introduced. The three generations are formed by the sequential breaking of the $\mathrm{SU}\left(N_{E T C}\right)$ gauge symmetry to the residual exact $\mathrm{SU}\left(N_{T C}\right)$. We denote the mass scale at which each stage of breaking takes place as $\Lambda_{i}$, where the $i$ 'th generation separates off from the other components of the ETC representations. 
A particularly attractive choice for the technicolor group, used in the explicit model to be studied here, is $\mathrm{SU}(2)_{T C}$, which minimizes the TC contributions to the $S$ parameter [9-11] and can yield walking behavior, allowing for enhanced quark and charged lepton masses. From Eq. (2.1), this choice of $N_{T C}=2$ implies that our ETC group is $\mathrm{SU}(5)_{\text {ETC }}$. With $N_{f} \simeq 16$ chiral technifermion doublets, as above, studies suggest that the $\mathrm{SU}(2)_{T C}$ theory could have an (approximate) infrared fixed point (IRFP). The theory would be in the confining phase with spontaneous chiral symmetry breaking, but near to the phase transition (as a function of $N_{f}$ ) beyond which it would go over into a nonabelian Coulomb phase $[12,13]$. This approximate IRFP provides walking behavior, enhancing the technifermion condensates that control the quark and charged lepton masses. This choice of $N_{T C}=2$ also plays a crucial role in our mechanism [6] for getting light neutrino masses and our present approach for explaining intra-generational mass splittings.

\section{B. Conventional ETC Mass Generation for Fermions}

The conventional ETC mechanism for the masses of quarks and charged leptons relates these masses to the TC condensate through ETC gauge boson exchange An estimate of the resultant masses is

$$
M_{i i}^{(f)} \simeq\left(\frac{g_{E T C}}{\sqrt{2}}\right)^{2} \frac{\eta\langle\bar{F} F\rangle}{M_{i}^{2}}
$$

where $i$ is the generation index, $\langle\bar{F} F\rangle \equiv\left\langle\sum_{i=4,5} \bar{F}_{i} F^{i}\right\rangle$ for a technifermion $F$ (sum on TC indices, no sum on color indices in the case where $F$ is a techniquark), $M_{i} \sim g_{E T C} \Lambda_{i}$ is the mass of the ETC gauge bosons that gain mass at scale $\Lambda_{i}$, where $g_{E T C}$ is the running ETC gauge coupling at this scale. The quantity $\eta$ is a renormalization group factor given by

$$
\eta=\exp \left[\int \frac{d \mu}{\mu} \gamma\left(\alpha_{T C}(\mu)\right)\right]
$$

reflecting the running of the bilinear operator $\bar{f} f$ between the technicolor scale and the relevant ETC scale, where $\gamma$ is the anomalous dimension for this operator. For a technicolor theory that exhibits full walking behavior between $\Lambda_{T C}$ and a scale denoted $\Lambda_{w}$, so that $\gamma \simeq 1$, it follows that

$$
\eta=\frac{\Lambda_{w}}{\Lambda_{T C}}
$$

Since only the $\mathrm{SU}(2)_{T C}$ theory is taken to walk, $\Lambda_{w}$ is the lowest ETC scale.

To evaluate Eq. (2.2), we use the approximate relation 


$$
\langle\bar{F} F\rangle=4 \pi f_{F}^{3}\left(\frac{3}{N_{T C}}\right)^{1 / 2},
$$

where $f_{F}$ is the technipion decay constant. We note that the corresponding QCD expression, $\langle\bar{q} q\rangle \simeq 4 \pi f_{\pi}^{3}\left(3 / N_{c}\right)^{1 / 2}$, is quite accurate. Here, $\langle\bar{q} q\rangle$ includes a sum over color for each quark $q$. With $f_{\pi}=93 \mathrm{MeV}$, one has a value of $(216 \mathrm{MeV})^{3}$ for $\langle\bar{q} q\rangle$. This may be compared to the current-algebra relation $f_{\pi}^{2} m_{\pi}^{2}=\left(m_{u}+m_{d}\right)\langle\bar{u} u+\bar{d} d\rangle=2\left(m_{u}+m_{d}\right)\langle\bar{q} q\rangle$, where $m_{u}$ and $m_{d}$ are the current quark masses and we have used the isospin symmetry of QCD to equate $\langle\bar{u} u\rangle=\langle\bar{d} d\rangle \equiv\langle\bar{q} q\rangle$. The values of the $u$ and $d$ current quark masses contain significant theoretical uncertainty; estimates range from $m_{u}+m_{d} \simeq 15 \mathrm{MeV}$ from older current algebra methods [15] to $9 \mathrm{MeV}$ using lattice methods [16]. Using the illustrative value $\left(m_{u}+m_{d}\right) \simeq 9$ $\mathrm{MeV}$, one finds $\langle\bar{u} u\rangle=\langle\bar{d} d\rangle=(211 \mathrm{MeV})^{3}$, in good agreement with the above.

Next we recall that for a technicolor theory with one SM family of technifermions,

$$
m_{W}^{2}=\frac{g^{2}}{4}\left(N_{c}+1\right) f_{F}^{2}
$$

where $g$ is the electroweak coupling and $f_{F}$ is the technipion decay constant. This yields $f_{F} \simeq$ $125 \mathrm{GeV}$. It is convenient to express various quantities in terms of the energy scale $\Lambda_{T C}$ at which the technicolor interaction gets sufficiently large to cause technifermion condensation. In QCD, $f_{\pi}=93 \mathrm{MeV}$ and $\Lambda_{Q C D} \simeq 180 \mathrm{MeV}$ so that $\Lambda_{Q C D} \simeq 2 f_{\pi}$; using this as a guide to technicolor, one infers $\Lambda_{T C} \simeq 250 \mathrm{GeV}$. If one regards $N_{c}=3$ and $N_{T C}=2$ as being sufficiently large so that one should include factors yielding the correct respective large$N_{c}$ and large- $N_{T C}$ behaviors, then one would write $\Lambda_{Q C D} \simeq 2 f_{\pi} \sqrt{3 / N_{c}}$ and hence $\Lambda_{T C} \simeq$ $2 f_{F} \sqrt{3 / N_{T C}} \simeq 300 \mathrm{GeV}$. We include these factors here, and hence use $\Lambda_{T C}=300 \mathrm{GeV}$.

Since the ETC theory is strongly coupled, we cannot calculate precisely the relation between $M_{i}$ and $\Lambda_{i}$. We take

$$
M_{i}=\frac{a g_{E T C} \Lambda_{i}}{4}
$$

where the constant $a$ is expected to be of order unity. Substituting in Eq. (2.2), we get

$$
M_{i i}^{(u)} \simeq \frac{\kappa \eta \Lambda_{T C}^{3}}{\Lambda_{i}^{2}}
$$

where $\kappa=8 \pi / 3 a^{2}(\simeq 8 \pi / 3$ with $a=O(1))$. This rough value for $\kappa$ will be used in all of our mass estimates.

Suppose that three separate ETC scales, $\Lambda_{1}>\Lambda_{2}>\Lambda_{3}$, emerge from the dynamical breaking. (This will be the case in the first of the two symmetry breaking sequences in the model to be described here.) Then, with the $\mathrm{SU}(2)_{T C}$ theory walking up to $\Lambda_{3}$, 


$$
M_{i i}^{(u)} \simeq \frac{\kappa \Lambda_{T C}^{2} \Lambda_{3}}{\Lambda_{i}^{2}} \quad i=1,2,3 .
$$

To compare this to the experimental values of the $Q=2 / 3$ quark masses, in particular, the top quark, we may neglect off-diagonal entries in $M^{(u)}$, at least for the higher two generations, so that the diagonal elements give the actual quark masses. With the ETC breaking scales

$$
\Lambda_{1} \simeq 10^{3} \mathrm{TeV}, \quad \Lambda_{2} \simeq 50 \mathrm{TeV}, \quad \Lambda_{3} \simeq 4 \mathrm{TeV}
$$

in the above formula (2.9), we get $m_{t} \simeq 175 \mathrm{GeV}, m_{c} \simeq 1.3 \mathrm{GeV}$ and $m_{u} \simeq 3 \mathrm{MeV}$ [17]. Since our ETC theory appears capable of generating the top quark mass, we shall not need to use other approaches (e.g. [18]- [19], [5]) for this purpose. The consistency of these choices of ETC scales with precision experimental constraints will be discussed in Section V.

Thus, while walking from $\Lambda_{T C}$ to the lowest ETC scale can successfully generate a sufficiently heavy top quark mass, as well as charm and up quark masses, this simple mechanism cannot be the entire story. It does not account, for example, for quark and charged lepton mass splittings within each generation. We next discuss this problem briefly, and introduce the mechanism designed to address it.

\section{Intra-generational Mass Splittings}

A longstanding challenge for dynamical theories of fermion masses has been to obtain the splittings $m_{t}>>m_{b}, m_{\tau}$ and $m_{c}>>m_{s}, m_{\mu}$ [20]. The fact that $U_{R}, D_{R}, E_{R}$, and $N_{R}$ have different hypercharges provides one source of splittings, and the fact that the techniquarks have color interactions while the technileptons do not provides another. However, these interactions are too weak at the scale $\Lambda_{T C}$, to explain the observed splittings without fine tuning.

The approach here is to assign right-handed components of the $Q=-1 / 3$ quarks and charged leptons to representations of the extended technicolor group that are conjugates of the representations assigned to the respective left-handed $Q=-1 / 3$ quarks and charged leptons, i.e.,

$$
\mathcal{R}\left(f_{L}\right)=\overline{\mathcal{R}}\left(f_{R}\right), \quad \text { for } f=d, e,
$$

retaining the conventional assignment

$$
\mathcal{R}\left(u_{L}\right)=\mathcal{R}\left(u_{R}\right)
$$

Here we use the notation $u_{L}, u_{R}, d_{L}, d_{R}, e_{L}, e_{R}$ (and, for the $\mathrm{SU}(2)_{L}$ doublets, $\left.Q_{L}, L\right)$ to refer to the full $\mathrm{SU}(5)_{E T C}$ representations with the indicated quantum numbers, encompassing in 
each case the three generations and the $N_{T C}=2$ technifermion components. Thus, for example, writing out $Q_{L}$ explicitly, we have

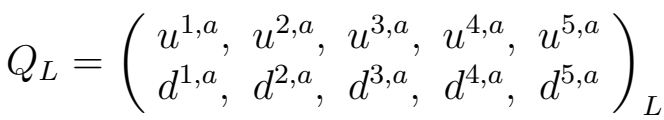

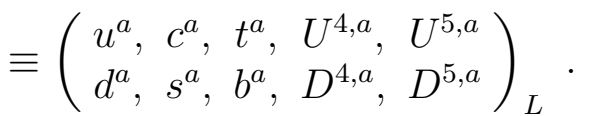

Note that we thus use synonymously the notation $f_{\chi}^{i} \equiv F_{\chi}^{i}$ for $i=4,5$ and $\chi=L, R$. Similarly, for leptons, we have, e.g.,

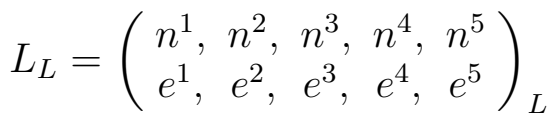

$$
\begin{aligned}
& \equiv\left(\begin{array}{ccccc}
\nu_{e}, & \nu_{\mu}, & \nu_{\tau}, & N^{4}, & N^{5} \\
e, & \mu, & \tau, & E^{4}, & E^{5}
\end{array}\right)_{L} .
\end{aligned}
$$

The condition (2.11) is similar to the device [3] employed to suppress Dirac neutrino masses in Refs. [6]. Note that this can only be done while maintaining a nonzero technicolor condensate if the technicolor group is $\mathrm{SU}(2)_{T C}$, corresponding here to the ETC group $\mathrm{SU}(5)_{E T C}$, since only in this case can one form a TC-gauge-invariant bilinear of the form $\left\langle\epsilon^{i j} \bar{F}_{i, L} F_{j, R}\right\rangle$ or $\left\langle\epsilon_{i j} \bar{F}_{L}^{i} F_{R}^{j}\right\rangle$, where $i, j$ are the SU(2) TC indices (and the color indices on the techniquarks are implicit). In this approach the diagrams giving rise to the masses of the $Q=-1 / 3$ quarks and charged leptons require mixing of ETC gauge bosons, which leads to strong suppression of these masses. In contrast, the masses of $Q=2 / 3$ quarks are generated in the conventional ETC manner, without any mixing of ETC gauge bosons. As we will show, this naturally produces large intra-generational mass splittings with acceptable violation of custodial $S U(2)$ symmetry. But in the models we have analyzed so far it leads to excessive suppression of the down-type quark and charged lepton masses.

\section{Quark Mixing}

Another challenge for (extended) technicolor models has been to generate the observed, inter-generational quark mixing. In general, the mass matrix $M^{(f)}(f=u, d, e)$ is diagonalized by a bi-unitary transformation

$$
M_{\text {diag. }}^{(f)}=U_{L}^{(f)} M^{(f)} U_{R}^{(f)}{ }^{\dagger}
$$

and the Cabibbo-Kobayashi-Maskawa [21] (CKM) quark mixing matrix $V$ defined by the charge-raising weak current 


$$
J_{\lambda}=\sum_{i, j=1}^{N_{g e n .}} \bar{u}_{i, L} V_{i j} \gamma_{\lambda} d_{L}^{j}
$$

is given by

$$
V=U_{L}^{(u)} U_{L}^{(d) \dagger}
$$

If both right and left-handed quarks are placed in the same representations (the fundamental or conjugate fundamental representation of $S U(5)_{E T C}$ in the present model), and there are no other interactions affecting the quark mass matrices, then it is not difficult to see that no quark mixing is produced. As the ETC group breaks sequentially, a set of massive ETC bosons is generated, with the $i$ 'th generation of quarks being connected to the corresponding techniquarks by the ETC gauge bosons $V_{t}^{i}, i \in\{1,2,3\}, t \in\{4,5\}$. If there is no mixing among the ETC bosons of the form $V_{t}^{i} \leftrightarrow V_{t}^{j}$ with $i \neq j$, then the only type of mass generation is that of Fig. 1. The mass matrix for both the up and down type quarks is diagonal, so there is no quark mixing.

To remedy this problem, the requisite combination of ETC gauge boson mixings must be generated. For the up-type quarks, with the conventional assignment 2.12, inter-generational mixing will exist, provided that

$$
V_{t}^{i} \leftrightarrow V_{t}^{j}, \quad i, j \in\{1,2,3\}, \quad i \neq j
$$

exists, where $t=4,5$ is a TC index. For the down-type quarks, with the unconventional assignment 2.11, inter-generational mixing will require the presence of

$$
V_{4}^{i} \leftrightarrow V_{j}^{5}, \quad V_{5}^{i} \leftrightarrow V_{j}^{4}, \quad i, j \in\{1,2,3\}
$$

In either case, the mass matrix will contain off-diagonal as well as diagonal elements. The differences between the off-diagonal elements in $M^{(u)}$ and $M^{(d)}$ and hence in the unitary transformations $U^{(u)}$ and $U^{(d)}$ then produce nontrivial quark mixing as specified in (2.17).

In the model to be described in Section IV of this paper, the breaking of the ETC gauge group is driven by a set of SM-singlet fermions, and this produces both sources for offdiagonal entries in mass matrices given in (2.18) and (2.19). The model takes $\mathcal{R}\left(u_{L}\right)=\mathcal{R}\left(u_{R}\right)$ to be the fundamental representation of $\mathrm{SU}(5)_{E T C}$. Graphs that produce the off-diagonal mass matrix elements for up-type quarks in this case are shown in Fig. 1. 


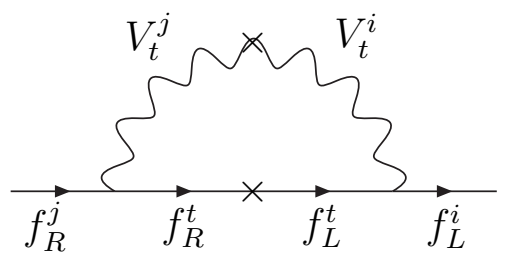

FIG. 1. Graphs generating $\bar{f}_{i, L} M_{i j}^{(f)} f_{R}^{j}$ where $i=1,2,3$, assuming that the indicated one-loop mixings of ETC gauge bosons occur, for the case in which $f_{L}$ and $f_{R}$ both transform according to the same (fundamental) representation of $\mathrm{SU}(5)_{E T C}$. The index $t$ takes on the values 4 and 5 . Here, $f^{i}$ is an up-type quark for $1 \leq i \leq 3$ and techniquark for $i=4,5$.

For the down-type quarks, the model of Section IV takes $\mathcal{R}\left(d_{L}\right)$ to be the fundamental representation of $\mathrm{SU}(5)_{E T C}$ and $\mathcal{R}\left(d_{R}\right)$ to be the conjugate fundamental representation. With $N_{T C}=2$, so that the $\mathrm{SU}(2)_{T C}$ condensates can still form, bilinears for these quarks arise via diagrams that necessarily involve ETC gauge boson mixing. The down-type techniquark condensate is of the form $\left\langle\epsilon^{i j} \bar{D}_{i, a, L} D_{j, R}^{a}\right\rangle$, where here $i, j$ and $a$ denote technicolor and color indices. This generates off-diagonal elements in $M^{(d)}$ via Eq. (2.19). The mass matrix for the down-type quarks, generically of the form

$$
\bar{d}_{i, L} M_{i j}^{(f)} d_{j, R}+\text { h.c. }
$$

is generated by the graph of Fig. 2. The indicated ETC mixing will be shown to exist in the model of Section IV.

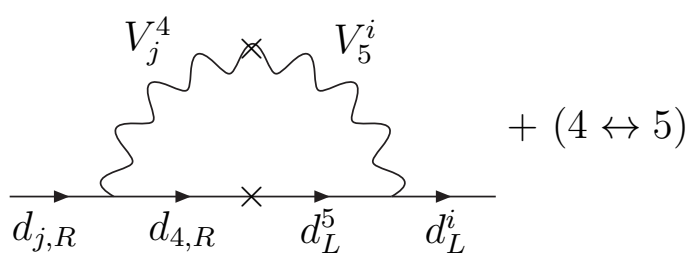

FIG. 2. Graphs generating $\bar{d}_{i, L} M_{i j}^{(d)} d_{j, R}$ where $i=1,2,3$, assuming that the indicated, one-loop mixings of ETC gauge bosons occur, for the case in which $d_{L}$ and $d_{R}$ transform according to the fundamental and conjugate fundamental representation of $\mathrm{SU}(5)_{E T C}$. Here $d^{i}$ is a down-type quark (techniquark) for $1 \leq i \leq 3$ $(i=4,5)$. As indicated, the graph with the indices 4 and 5 interchanged also contributes. 
For the charged leptons, $e$, there are two possibilities. The $e$ fields of a given chirality $\chi=L, R$ can transform according to the same $(\mathrm{S})$ or conjugate $(\mathrm{C})$ ETC representations as the down-type quarks and techniquarks, $d$. We have considered both possibilities, and we label the two classes of models as DES and DEC, respectively. The model of Section IV will be of the DEC class, with $\mathcal{R}\left(e_{L}\right)$ taken to be the conjugate fundamental representation of $\mathrm{SU}(5)_{E T C}$, and $\mathcal{R}\left(e_{R}\right)$ taken to be the fundamental representation.

In this model then, the technifermion condensates take the following forms:

$$
\left\langle\epsilon^{i j} \bar{D}_{i, a, L} D_{j, R}^{a}\right\rangle, \quad\left\langle\epsilon_{i j} \bar{E}_{L}^{i} E_{R}^{j}\right\rangle
$$

(together with their hermitian conjugates) where, as before, $i, j$ are technicolor indices and $a$ are color indices. Since the representations of $\mathrm{SU}(2)$ are (pseudo)real, the technicolor interaction, by itself, would produce the same magnitude for all of the technifermion condensates, both the ones in Eq. (2.21) and the $\left\langle\bar{U}_{i, a, L} U_{L}^{i, a}\right\rangle$ (for each color, in the case of techniquarks). Diagrams analogous to those in Fig. 2, with obvious changes in the DEC case, give rise to the charged-lepton mass matrix $M_{i j}^{(e)}$.

This mechanism will lead to off-diagonal mass matrix elements for the down-type quarks and charged leptons that are different from those of the up-type quarks. It can therefore lead to CKM mixing among the quarks and explain why $m_{t}>>m_{b}, m_{\tau}$ and $m_{c}>>m_{s}, m_{\mu}$. The same mechanism has been used to suppress the elements of Dirac mass matrices for the neutrinos $[3,22]$, providing an ingredient in a possible new type of seesaw mechanism. This will be discussed in Section IV.

\section{PROGENITORS}

The model to be described in Section IV evolved from two earlier models, designated here as AT94 [22] and AS02 [6]. In addition, an embedding of AS02 in models with an extended standard model gauge group was described in Refs. [7,8]. Since these models use the same representation for the left- and right-handed components of all the quarks and charged leptons, they do not lead to nontrivial quark mixings. We review their ingredients briefly to set the stage for the model of Section IV.

A basic condition for building any of these models is the cancellation of the $\mathrm{SU}(5)_{E T C}$ gauge anomaly. The SM-nonsinglet fermions and technifermions contribute the following terms to this anomaly (written for right-handed chiral components): $A\left(Q_{R}^{c}\right)=-2 N_{c}=-6$, $A\left(L_{R}^{c}\right)=-2, A\left(u_{R}\right)=A\left(d_{R}\right)=N_{c}=3$, and $A\left(e_{R}\right)=1$, for a total of -1 . It follows that the contributions of the right-handed SM-singlet fermions must be 


$$
\sum_{f_{R}} A\left(f_{R}\right)=1
$$

We describe below the ingredients of each of these models. The breaking patterns are discussed in the original references. We then note that the incorporation of Pati-Salam symmetry, used in two of these models, is not compatible with the approach of the present paper.

\section{A. AT94}

In Ref. [22] an ETC model was constructed that explicitly demonstrated the sequential self-breaking of the ETC group, producing three generations of SM fermions. This model was based on the gauge group

$$
G_{A T 94}=\mathrm{SU}(5)_{E T C} \times \mathrm{SU}(2)_{H C} \times \mathrm{SU}(4)_{P S} \times \mathrm{SU}(2)_{L} \times \mathrm{U}(1)_{R}
$$

where $\mathrm{SU}(4)_{P S}$ is the Pati-Salam group, which gauges baryon minus lepton number $U(1)_{B-L}$ and combines it with color $\mathrm{SU}(3)_{c}$. This yields quantization of electric charge and partial unification of quarks and leptons. The $\mathrm{U}(1)_{R}$ is an $R$-charge abelian group. Thus the gauge group involves two gauge interactions that become strong at high energies: extended technicolor $\mathrm{SU}(5)_{E T C}$, and one additional strong gauge interaction called hypercolor (HC), $\mathrm{SU}(2)_{H C}$. The latter is included in order to produce the desired sequential gauge symmetry breaking.

The fermions in this theory are listed below, in a notation where the numbers indicate the representations under $\mathrm{SU}(5)_{E T C} \times \mathrm{SU}(2)_{H C} \times \mathrm{SU}(4)_{P S} \times \mathrm{SU}(2)_{L}$, and the subscript gives the $\mathrm{U}(1)_{R}$ charge:

$$
\begin{aligned}
(5,1,4,2)_{0, L}, \quad & (5,1,4,1)_{1, R}, \quad(5,1,4,1)_{-1, R} \\
& (1,1,6,1)_{0, R}, \quad(1,2,6,1)_{0, R} \\
& (\overline{5}, 1,1,1)_{0, R}, \quad(\overline{10}, 1,1,1)_{0, R}, \quad(10,2,1,1)_{0, R}
\end{aligned}
$$

(In [22] these were written equivalently in left-handed holomorphic form.)

A sequential breaking pattern was described in AT94, yielding some of the features of the quark and lepton masses. The (Dirac) neutrino masses, although much smaller than the quark and charged lepton masses, were still much too large. 


\section{B. AS02}

In Ref. [6] it was shown how one could get realistically light neutrinos in a model with dynamical electroweak symmetry breaking. The model used the ETC and HC gauge groups of Ref. [22], together with only the SM gauge group:

$$
G=\mathrm{SU}(5)_{E T C} \times \mathrm{SU}(2)_{H C} \times G_{S M}
$$

where

$$
G_{S M}=\mathrm{SU}(3) \times \mathrm{SU}(2)_{L} \times \mathrm{U}(1)_{Y}
$$

The fermion content of this model is listed below, where the numbers indicate the representations under $\mathrm{SU}(5)_{E T C} \times \mathrm{SU}(2)_{H C} \times \mathrm{SU}(3)_{c} \times \mathrm{SU}(2)_{L}$ and the subscript gives the weak hypercharge $Y$ :

$$
\begin{array}{ll}
(5,1,3,2)_{1 / 3, L}, \quad & (5,1,3,1)_{4 / 3, R}, \quad(5,1,3,1)_{-2 / 3, R} \\
(5,1,1,2)_{-1, L}, \quad & (5,1,1,1)_{-2, R}, \\
& (\overline{10}, 1,1,1)_{0, R}, \quad(10,2,1,1)_{0, R} .
\end{array}
$$

Two sequential breaking patterns were studied in Ref. [6].

\section{Models with Extended Strong-Electroweak Gauge Groups}

Refs. $[7,8]$ enlarged the model of Ref. [6] by embedding the SM gauge theory in two models with an extended strong-electroweak gauge group. These, too, are broken dynamically. The first is based on the group

$$
G=\mathrm{SU}(5)_{E T C} \times \mathrm{SU}(2)_{H C} \times G_{L R}
$$

where

$$
G_{L R}=\mathrm{SU}(3)_{c} \times \mathrm{SU}(2)_{L} \times \mathrm{SU}(2)_{R} \times \mathrm{U}(1)_{B-L} .
$$

The fermion content is

$$
(5,1,3,2,1)_{1 / 3, L}, \quad(5,1,3,1,2)_{1 / 3, R},
$$




$$
\begin{aligned}
& (5,1,1,2,1)_{-1, L}, \quad(5,1,1,1,2)_{-1, R}, \\
& (\overline{5}, 1,1,1,1)_{0, R}, \quad(\overline{10}, 1,1,1,1)_{0, R}, \quad(10,2,1,1,1)_{0, R} .
\end{aligned}
$$

The second uses the group

$$
G=\mathrm{SU}(5)_{E T C} \times \mathrm{SU}(2)_{H C} \times G_{422}
$$

where

$$
G_{422}=\mathrm{SU}(4)_{P S} \times \mathrm{SU}(2)_{L} \times \mathrm{SU}(2)_{R} .
$$

and $\mathrm{SU}(4)_{P S}$ is the Pati-Salam group. This achieves a higher degree of unification, as compared with (3.7). Here the fermion content is

$$
\begin{aligned}
& (5,1,4,2,1)_{L}, \quad(5,1,4,1,2)_{R}, \\
& (\overline{5}, 1,1,1,1)_{R}, \quad(\overline{10}, 1,1,1,1)_{R}, \quad(10,2,1,1,1)_{R} .
\end{aligned}
$$

These two models explain how $G_{L R}$ and $G_{422}$ break to $\mathrm{SU}(3)_{c} \times \mathrm{SU}(2)_{L} \times \mathrm{U}(1)_{Y}$, and why this takes place at a scale $\left(\simeq 10^{3} \mathrm{TeV}\right)$ large compared to the electroweak scale, but much smaller than a GUT scale. We note, however, that they are not compatible with the approach of the present paper where the left- and right-handed components of the $Q=$ $-1 / 3$ quarks and charged leptons are placed in conjugate ETC representations (Eq. (2.11)). The $\mathrm{SU}(2)_{L} \times \mathrm{SU}(2)_{R}$ symmetry would imply that $\mathcal{R}\left(u_{L, R}\right)=\mathcal{R}\left(d_{L, R}\right)$. Since the choice $\mathcal{R}\left(u_{L}\right)=\mathcal{R}\left(u_{R}\right)$ is necessary to provide conventional mass ETC mass generation for the $Q=2 / 3$ quarks, the use of conjugate representations for the $Q=-1 / 3$ quarks would not be allowed. The inclusion of $\mathrm{SU}(4)_{P S}$ symmetry as in $G_{422}$ would also prohibit the use of conjugate representations for the charged leptons.

\section{A MODEL FOR QUARKS AND LEPTONS}

\section{A. Ingredients}

We employ the same gauge group $\mathrm{G}$ as in Ref. [6], given in Eq. (3.4), and use relatively conjugate ETC representations for the left- and right-handed components of $Q=-1 / 3$ quarks and charged leptons. All of the nonabelian factor groups in $G$ are asymptotically free. There are no bilinear fermion operators invariant under $G$ and hence there are no bare 
fermion mass terms. The $\mathrm{SU}(2)_{T C}$ subsector of $\mathrm{SU}(5)_{E T C}$, and the $\mathrm{SU}(2)_{H C}$ interaction, are vectorial. The representation content for the quarks is

$$
Q_{L}:(5,1,3,2)_{1 / 3, L}, \quad u_{R}:(5,1,3,1)_{4 / 3, R}, \quad d_{R}(\overline{5}, 1,3,1)_{-2 / 3, R} .
$$

The representations for the charged leptons are

$$
L_{L}:(\overline{5}, 1,1,2)_{-1, L}, \quad e_{R}:(5,1,1,1)_{-2, R}
$$

so the model is of DEC type (Eq. (2.11)).

The model also contains SM-singlet fermions, constrained by the absence of an $\mathrm{SU}(5)_{E T C}$ gauge anomaly. We denote the contribution of a given chiral fermion $f_{R}$, written as a righthanded field, to this anomaly, as $A\left(f_{R}\right)$. The quarks and techniquarks make the contributions $A\left(Q_{R}^{c}\right)=-2 N_{c}=-6, A\left(u_{R}\right)=-A\left(d_{R}\right)=N_{c}=3$. In a DEC-type model, $A\left(L_{R}^{c}\right)=$ $2, A\left(e_{R}\right)=1$, while for a DES-type model, $A\left(L_{R}^{c}\right)=-2, A\left(e_{R}\right)=-1$. The totals for the contributions from these SM fermions are -3 and -9 for models of DEC and DES type, respectively. In order that a model should have zero $\mathrm{SU}(5)_{E T C}$ gauge anomaly, the contributions of the right-handed SM-singlet fermions to this anomaly must be

$$
\sum_{\text {SM-singlet } f_{R}} A\left(f_{R}\right)= \begin{cases}3 & \text { for DEC-type models } \\ 9 & \text { for DES-type models }\end{cases}
$$

Since $\mathrm{SU}(2)_{H C}$ is used for the hypercolor group, it is free of any local gauge anomaly; the constraint of no global anomaly requires that there be an even number of doublets, and this is satisfied for our model, since it has hypercolored fermions transforming as a 10 (antisymmetric rank-2 tensor) or SU(5)ETC and two doublets under $\mathrm{SU}(2)_{H C}$.

There are a variety of solutions to the constraint Eq. (4.3), and we have studied a number of these. Here we focus on one relatively simple solution. For the SM-singlet fermions we take

$$
\psi_{R}^{i j}:(10,1,1,1)_{0, R}, \quad \zeta_{R}^{i j, \alpha}:(10,2,1,1)_{0, R}, \quad \omega_{p, R}^{\alpha}: 2(1,2,1,1)_{0, R}
$$

where $1 \leq i, j \leq 5$ are $\mathrm{SU}(5)_{E T C}$ indices, $\alpha=1,2$ are $\mathrm{SU}(2)_{H C}$ indices, and $p=1,2$ refers to the two copies of the $\omega_{p, R}^{\alpha}$ field. The SM-singlet fermions in this model thus involve three types: (i) ETC-nonsinglet, HC-singlets, viz., the antisymmetric rank-2 tensor $\psi_{R}^{i j}$; (ii) ETCnonsinglet, HC-nonsinglet, viz., the $\zeta_{R}^{i j, \alpha}$ field, which transforms as an antisymmetric rank-2 tensor representation of $\mathrm{SU}(5)_{E T C}$ and a fundamental representation of $\mathrm{SU}(2)_{H C}$, and (iii) the ETC-singlet, HC-nonsinglet fields $\omega_{p, R}^{\alpha}$, which transform as fundamental representations of $\mathrm{SU}(2)_{H C}$ (we include an even number $p=2$ of copies to avoid a global $\mathrm{SU}(2)_{H C}$ anomaly). 
The subsector comprised of the $\psi_{R}^{i j}$ and $\zeta_{R}^{i j, \alpha}$ is the same as the SM-singlet sector of the model discussed in Ref. [6]. One can see that condition (4.3) is satisfied and, more generally, that this model is free of any gauge and global anomalies.

In a theory in which lepton number is not gauged, it is a convention how one assigns the lepton number $L$ of the SM-singlet fields. We assign $L=1$ to $\psi_{R}^{i j}$ so that the Dirac mass terms $\bar{n}_{L}^{i} b_{i j} \psi_{R}^{1 j}$ that will form conserve $L$. One could, alternatively, assign $L=0$ to $\psi_{R}^{i j}$, so that the Dirac mass terms transform as $\Delta L=1$. Indeed, in the models of Refs. [7,8], where $L$ is gauged, this is the way that the $\Delta L=2$ violation of lepton number arises. The lepton number assigned to the $\zeta_{R}^{i j, \alpha}$ and $\omega_{R}^{\alpha}$ is also a convention; since they have no Dirac terms with the electroweak-doublet neutrinos, we leave these assignments arbitrary.

\section{B. Symmetry Breaking - The First Stage}

Symmetry breaking down to the TC scale is driven completely by the SM-singlet sector of the model. We identify plausible preferred condensation channels using a generalizedmost-attractive-channel (GMAC) approach that takes account of one or more strong gauge interactions at each breaking scale, as well as the energy cost involved in producing gauge boson masses when gauge symmetries are broken. An approximate measure of the attractiveness of a channel $R_{1} \times R_{2} \rightarrow R_{\text {cond. }}$ is taken to be $\Delta C_{2}=C_{2}\left(R_{1}\right)+C_{2}\left(R_{2}\right)-C_{2}\left(R_{\text {cond. }}\right)$, where $R_{j}$ denotes the representation under a relevant gauge interaction and $C_{2}(R)$ is the quadratic Casimir invariant.

As the energy decreases from some high value, the $\mathrm{SU}(5)_{E T C}$ and $\mathrm{SU}(2)_{H C}$ couplings increase. We envision that at $E \simeq \Lambda_{1} \gtrsim 10^{3} \mathrm{TeV}, \alpha_{E T C}$ is sufficiently strong [13] to produce condensation in the channel

$$
(10,1,1,1)_{0, R} \times(10,1,1,1)_{0, R} \rightarrow(\overline{5}, 1,1,1)_{0}
$$

with $\Delta C_{2}=24 / 5=4.8$, breaking $\mathrm{SU}(5)_{E T C} \rightarrow \mathrm{SU}(4)_{E T C}$. With no loss of generality, we take the breaking direction in $\mathrm{SU}(5)_{E T C}$ as $i=1$; this entails the separation of the first generation of quarks and leptons from the components of $\mathrm{SU}(5)_{E T C}$ fields with indices lying in the set $\{2,3,4,5\}$. With respect to the unbroken $\mathrm{SU}(4)_{E T C}$, we have the decomposition $(10,1,1,1)_{0, R}=(4,1,1,1)_{0, R}+(6,1,1,1)_{0, R}$. We denote the fundamental representation $(4,1,1,1)_{0, R}$ and antisymmetric tensor representation $(6,1,1,1)_{0, R}$ as $\alpha_{R}^{1 i} \equiv \psi_{R}^{1 i}$ for $2 \leq i \leq 5$ and $\xi_{R}^{i j} \equiv \psi_{R}^{i j}$ for $2 \leq i, j \leq 5$. The associated $\mathrm{SU}(5)_{E T C^{-}}$-breaking, $\mathrm{SU}(4)_{E T C^{-}}$-invariant condensate is then

$$
\left\langle\epsilon_{1 i j k \ell} \xi_{R}^{i j}{ }^{T} C \xi_{R}^{k \ell}\right\rangle=8\left\langle\xi_{R}^{23}{ }^{T} C \xi_{R}^{45}-\xi_{R}^{24}{ }^{T} C \xi_{R}^{35}+\xi_{R}^{25}{ }^{T} C \xi_{R}^{34}\right\rangle
$$


This condensate and the resultant dynamical Majorana mass terms of order $\Lambda_{1}$ for the six $\xi_{R}^{i j}$ fields in Eq. (4.6) violate total lepton number as $|\Delta L|=2$. The actual mass eigenstates are linear combinations of these six fields involving maximal $( \pm \pi / 4)$ mixing of $\left|\xi_{24, R}\right\rangle$ with $\left|\xi_{35, R}\right\rangle,\left|\xi_{34, R}\right\rangle$ with $\left|\xi_{25, R}\right\rangle$, and $\left|\xi_{23, R}\right\rangle$ with $\left|\xi_{45, R}\right\rangle$, respectively, with respective eigenvalues $\pm \Lambda_{1}$ (where in the case of negative eigenvalues, one redefines fields appropriately so as to obtain positive masses).

At energy scales below $\Lambda_{1}$, depending on relative strengths of couplings, various symmetry-breaking sequences with different condensates are plausible. As all the condensates develop, there will naturally arise relative phases among them, leading in general to the presence of $\mathrm{CP}$ violation in both the quark and neutrino sectors. We postpone an analysis of these phases and of $\mathrm{CP}$ violation from topological terms in the gauge sector and the associated strong CP problem for later work, concentrating in this paper on the magnitudes of the condensates and the resultant pattern of masses and mixing angles.

\section{Neutrino Mass Matrix}

Having described the SM-singlet sector of our model and the first stage of symmetry breaking at scale $\Lambda_{1}$, we are now in a position to discuss the general structure of the neutrino mass matrix and the possibility of a seesaw mechanism. The details will then depend on which lower-energy symmetry breaking sequence is favored.

The full neutrino mass term for the model is

$$
-\mathcal{L}_{m}=\frac{1}{2}\left(\bar{n}_{L}{\overline{\chi^{c}}}_{L}\right)\left(\begin{array}{cc}
M_{L} & M_{D} \\
\left(M_{D}\right)^{T} & M_{R}
\end{array}\right)\left(\begin{array}{c}
n_{R}^{c} \\
\chi_{R}
\end{array}\right)+\text { h.c. }
$$

where $n_{L}=\left(\nu_{e}, \nu_{\mu}, \nu_{\tau}, N_{4}, N_{5}\right)_{L}$ and $\chi_{R}$ is a vector of the $n_{s}$ SM-singlet fields, including $\alpha_{R}^{i j}, \xi_{R}^{i j}, \zeta_{R}^{i j, \alpha}$, and $\omega_{p, R}^{\alpha}$. Since $\left(M_{L}\right)^{T}=M_{L}$ and $\left(M_{R}\right)^{T}=M_{R}$, the full $\left(5+n_{s}\right) \times\left(5+n_{s}\right)$ neutrino mass matrix $M$ in (4.7) is complex symmetric and can be diagonalized by a unitary transformation $U_{\nu}$ as

$$
M_{\text {diag. }}=U_{\nu}^{\dagger} M\left(U_{\nu}^{\dagger}\right)^{T}
$$

This yields the neutrino masses and transformation $U_{\nu}$ relating the group eigenstates $\nu_{L}=$ $\left(\bar{n}, \overline{\chi^{c}}\right)_{L}^{T}$ and the corresponding mass eigenstates $\nu_{m, L}$, according to

$$
\nu_{j, L}=\sum_{k=1}^{5+n_{s}}\left(U_{\nu}\right)_{j k} \nu_{k, m, L}, \quad 1 \leq j \leq 5+n_{s}
$$


(the elements $\left(U_{\nu}\right)_{j k}$ connecting techni-singlet and technicolored neutrinos vanish identically). The lepton mixing matrix for the observed neutrinos $[23,24] \nu_{\ell, L}=U \nu_{m, L}$ is then given by

$$
U_{i k}=\sum_{j=1}^{3}\left(U_{\ell, L}\right)_{i j}\left(U_{\nu}\right)_{j k}, \quad 1 \leq i \leq 3, \quad 1 \leq k \leq 5+n_{s}
$$

where $U_{1 k} \equiv U_{e k}$, etc., and where the diagonalization of the charged lepton mass matrix is carried out by the bi-unitary transformation in Eq. (2.15) for $f=e$. Thus, the chargelowering leptonic weak current is given by

$$
J^{\lambda}=\sum_{i=1}^{3} \sum_{k=1}^{5+n_{s}} \bar{e}_{L}^{i} \gamma^{\lambda} U_{i k}\left(\nu_{m}\right)_{k, L}
$$

where $e_{i}$ denotes the $i$ th charged lepton mass eigenstate and $\nu_{m}$ is the $\left(5+n_{s}\right)$-dimensional vector of neutrino mass eigenstates.

The complex symmetric mass matrix $M$ of neutrino-like (colorless and electrically neutral) states is $39 \times 39$, with $n_{s}=34$. Of the 39 neutrino-like chiral components of fermion fields, $N_{\text {gen }}=3$ are the observed left-handed neutrinos, $N_{T C}=2$ are left-handed technineutrinos, and the other $n_{s}=34$ are electroweak-singlets, comprised of the $10(\overline{10}, 1,1,1)_{0, R}$, the $20(10,2,1,1)_{0, R}$, and the four fermions in the pair of $(1,2,1,1)_{0, R}$. All of its entries arise as the high-energy physics is integrated out at each stage of condensation from $\Lambda_{1}$ down to $\Lambda_{T C}$. Composite operators of various dimension are formed, with bilinear condensation then leading to the masses. The nonzero entries of $M$ arise either directly, as dynamical masses associated with various condensates, or via loop diagrams involving dynamical mass insertions on internal fermion lines with, in most cases, mixings among ETC gauge bosons on internal lines. The different origins for the elements of $M$ give rise to quite different magnitudes for these elements; in particular, there is substantial suppression of the second class because the diagrams involve ratios of small scales such as $\Lambda_{T C}$ and lower ETC scales to larger scales such as $\Lambda_{1}$.

In the two symmetry breaking sequences to be considered here, either the $\mathrm{SU}(2)_{H C}$ symmetry or a $\mathrm{U}(1)_{H C}$ subgroup will remain unbroken. Therefore, the hypercolored fermions will not form bilinear condensates and resultant mass terms with hypercolor-singlet fermions. Hence, $M$ has a block-diagonal structure, and it is convenient to group the HC-singlet blocks together as $M_{H C S}$ and the HC-nonsinglet blocks together as $M_{H C}$. The matrix of primary interest, $M_{H C S}$, is defined by the operator product

$$
-\mathcal{L}_{H C S}=\frac{1}{2}\left(\bar{n}_{L},{\overline{\alpha^{c}}}_{L}, \bar{\xi}_{L}\right) M_{H C S}\left(\begin{array}{c}
n_{R}^{c} \\
\alpha_{R} \\
\xi_{R}
\end{array}\right)+\text { h.c. }
$$


so that

$$
M_{H C S}=\left(\begin{array}{ccc}
M_{L} & \left(M_{D}\right)_{\bar{n} \alpha} & \left(M_{D}\right)_{\bar{n} \xi} \\
\left(M_{D}\right)_{\bar{n} \alpha}^{T} & \left(M_{R}\right)_{\alpha \alpha} & \left(M_{R}\right)_{\alpha \xi} \\
\left(M_{D}\right)_{\bar{n} \xi}^{T} & \left(M_{R}\right)_{\alpha \xi}^{T} & \left(M_{R}\right)_{\xi \xi}
\end{array}\right)
$$

This matrix has many vanishing entries arising from the fact that the $S U(2)_{T C}$ symmetry is exact. It, too, could therefore be written in a block-diagonal form by clustering the seven TC-singlet fermions, $\left(\left(n^{c}\right)^{1},\left(n^{c}\right)^{2},\left(n^{c}\right)^{3}, \alpha^{12}, \alpha^{13}, \xi^{23}, \xi^{45}\right)_{R} \equiv\left(\nu_{e}^{c}, \nu_{\mu}^{c}, \nu_{\tau}^{c}, \alpha^{12}, \alpha^{13}, \xi^{23}, \xi^{45}\right)_{R}$, in one group, and the TC-doublet fermions is a second group. This block-diagonal structure will be evident in many of the exact zeros in the various matrices displayed below.

Within $M_{H C S}$, the most important Dirac submatrix is $\left(M_{D}\right)_{\bar{n} \alpha}$, defined by the operator product

$$
\bar{n}_{L}^{i}\left[\left(M_{D}\right)_{\bar{n} \alpha}\right]_{i j} \alpha_{R}^{1 j}
$$

with $1 \leq i \leq 5,2 \leq j \leq 5$. This matrix has the general form

$$
\left(M_{D}\right)_{\bar{n} \alpha}=\left(\begin{array}{cccc}
b_{12} & b_{13} & 0 & 0 \\
b_{22} & b_{23} & 0 & 0 \\
b_{32} & b_{33} & 0 & 0 \\
0 & 0 & 0 & c_{1} \\
0 & 0 & -c_{1} & 0
\end{array}\right) .
$$

The vanishing entries are zero because of exact technicolor gauge invariance. The entry $c_{1}$ represents a dynamical mass directly generated by technicolor interactions corresponding to

$$
\sum_{i, j=4,5} \epsilon_{i j} \bar{n}_{L}^{i} \alpha_{R}^{1 j}
$$

so that

$$
\left|c_{1}\right| \simeq \Lambda_{T C}
$$

In Fig. 3 we show graphs that contribute to $\bar{n}_{L}^{i} b_{i j} \alpha_{R}^{1 j}$ for $i=1,2,3$ and $j=2,3$ if the indicated mixings of ETC gauge bosons occur. Which of these ETC gauge boson mixings do occur depends on the symmetry-breaking sequence.

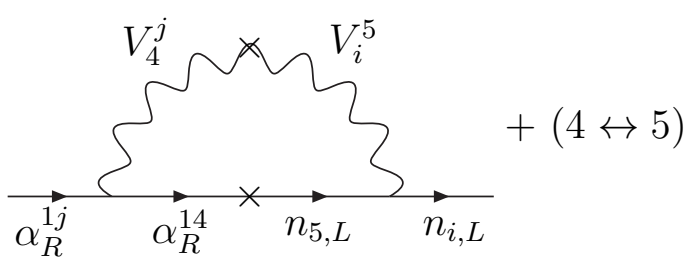


FIG. 3. Graphs generating $\bar{n}_{L}^{i} b_{i j} \alpha_{R}^{1 j}$ for $i=1,2,3$ and $j=2,3$, provided that the indicated mixings of ETC gauge bosons occur.

The sub-matrix $\left(M_{R}\right)_{\alpha \alpha}$, which, depending on mass scales, can play a key role in a seesaw mechanism [6], is associated with the operator product

$$
\left(\overline{\alpha^{c}}\right)_{L}^{1 i} r_{i j} \alpha_{R}^{1 j}=\alpha_{R}^{1 i}{ }^{T} C r_{i j} \alpha_{R}^{1 j}
$$

With the usual ordering of the components in the 4 of SU(4) ETC, viz., $\left(\alpha^{12}, \alpha^{13}, \alpha^{14}, \alpha^{15}\right)$, this matrix is

$$
\left(M_{R}\right)_{\alpha \alpha}=\left(\begin{array}{cccc}
r_{22} & r_{23} & 0 & 0 \\
r_{23} & r_{33} & 0 & 0 \\
0 & 0 & 0 & 0 \\
0 & 0 & 0 & 0
\end{array}\right)
$$

As before, the zeros are exact due to technicolor invariance. If the $2 \times 2 r_{i j}$ submatrix has maximal rank, it could provide the seesaw which, in conjunction with the suppression of the Dirac entries $b_{i j}$ discussed above, could yield adequate suppression of neutrino masses. The $r_{i j}$ submatrix plays this role because $\alpha_{12, R}$ and $\alpha_{13, R}$ are the electroweak-singlet technisinglet neutrinos that remain as part of the low-energy effective theory below the electroweak scale. In Fig. 4 we show graphs that contribute to $r_{23}$.

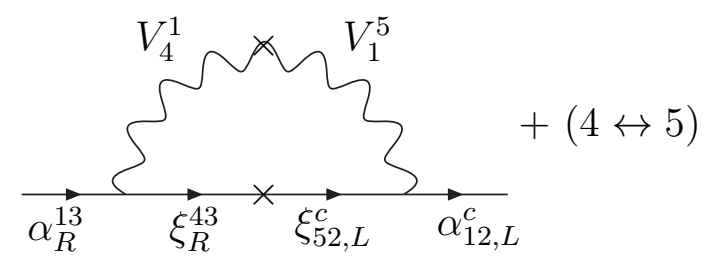

FIG. 4. Graphs for $\alpha_{R}^{12}{ }^{T} C r_{23} \alpha_{R}^{13}$.

The other submatrices in $M$ do not have as important an effect on the primary mass eigenstates in the observed neutrinos. The second Dirac submatrix in Eq. $(4.13),\left(M_{D}\right)_{\bar{n} \xi}$ is associated with the operator product

$$
\bar{n}_{L}^{i}\left[\left(M_{D}\right)_{\bar{n} \xi}\right]_{i, k n} \xi_{R}^{k n}
$$

with $1 \leq i \leq 5$ and $2 \leq k<n \leq 5$ and has the general form 


$$
\left(M_{D}\right)_{\bar{n} \xi}=\left(\begin{array}{cccccc}
d_{1,23} & d_{1,45} & 0 & 0 & 0 & 0 \\
d_{2,23} & d_{2,45} & 0 & 0 & 0 & 0 \\
d_{3,23} & d_{3,45} & 0 & 0 & 0 & 0 \\
0 & 0 & 0 & c_{2} & 0 & c_{3} \\
0 & 0 & -c_{2} & 0 & -c_{3} & 0
\end{array}\right)
$$

Again, the zeros are exact and follow from technicolor invariance. Because the $\xi$ fields decouple from the theory at scales below $\Lambda_{1}$, they cannot directly condense with the $n$ fields at lower scales. Thus, the nonzero elements of $\left(M_{D}\right)_{\bar{n} \xi}$ arise only indirectly, via loop diagrams and are highly suppressed. These elements of $\left(M_{D}\right)_{\bar{n} \xi}$ have only a small effect on the neutrino eigenvalues because in the characteristic polynomial for the full neutrino mass matrix $M$, they occur as corrections to much larger terms involving $\Lambda_{1}$.

In Fig. 5(a,b) we show the one-loop diagrams that contribute to $\bar{n}_{L}^{i} d_{i, j k} \xi_{R}^{j k}$ for $j, k=4,5$ and (a) $i=1$, i.e., to $\bar{\nu}_{e, L} d_{1,45} \xi_{R}^{45}$ and (b) $i=2,3$, i.e., to $\bar{\nu}_{\mu, L} d_{2,45} \xi_{R}^{45}$ and $\bar{\nu}_{\tau, L} d_{3,45} \xi_{R}^{45}$. For the $i=1$ case shown in Fig. 5(a), no ETC gauge boson mixing is necessary, but for the $i=2,3$ cases shown in Fig. 5(b), one needs the mixings $V_{1}^{t} \rightarrow V_{i}^{t}$ for $t=4,5$.

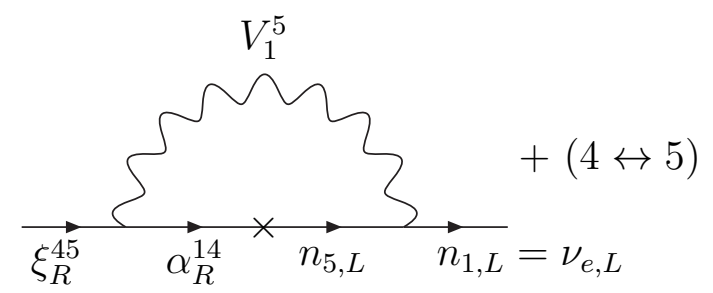

(a)

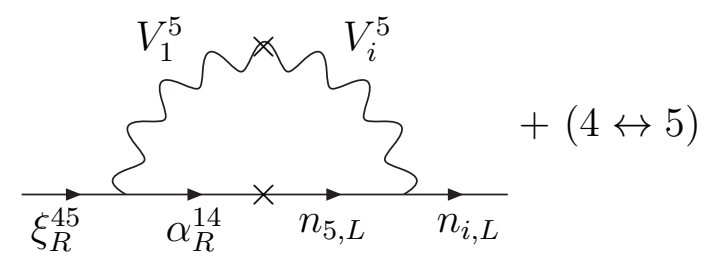

(b)

FIG. 5. Graphs that yield contributions to the Dirac bilinear $\bar{n}_{L}^{i} d_{i, j k} \xi_{R}^{j k}$ for $j, k=4,5$ and (a) $i=1$, (b) $i=2,3$. In the latter case, the mixing of ETC gauge bosons that is necessary is indicated.

The entries $c_{2}$ and $c_{3}$ in the matrix (4.21) correspond to the bilinears

$$
\sum_{i, j=4,5} \epsilon^{i j} \bar{n}_{i, L} \xi_{2 j, R}, \quad \sum_{i, j=4,5} \epsilon^{i j} \bar{n}_{i, L} \xi_{3 j, R}
$$


and are allowed by technicolor invariance, but they cannot occur directly as TC-scale dynamical masses since the $\xi$ fields, gain masses of order $\Lambda_{1}$ from the condensate (4.6). They might be induced by higher-order processes, but we will not pursue this in detail, since they are not important for the light neutrinos.

The submatrix $\left(M_{R}\right)_{\alpha \xi}$ corresponding to the bilinear

$$
{\overline{\alpha^{c}}}_{L}^{1 i} w_{1 i, j k} \xi_{R}^{j k}=\alpha_{R}^{1 i T} C w_{1 i, j k} \xi_{R}^{j k}
$$

has the general form

$$
\left(M_{R}\right)_{\alpha \xi}=\left(\begin{array}{cccccc}
w_{12,23} & w_{12,45} & 0 & 0 & 0 & 0 \\
w_{13,23} & w_{13,45} & 0 & 0 & 0 & 0 \\
0 & 0 & 0 & c_{4} & 0 & c_{5} \\
0 & 0 & -c_{4} & 0 & -c_{5} & 0
\end{array}\right)
$$

Again, all zeros in these matrices are exact and follow from $\mathrm{SU}(2)_{T C}$ invariance. In Eq. (4.24) the $\pm c_{4}$ and $\pm c_{5}$ entries refer to bilinear fermion operator products that are allowed by the exact $\mathrm{SU}(2)_{T C}$ invariance, namely

$$
\sum_{i, j=4}^{5} \epsilon_{123 i j}{\overline{\alpha^{c}}}_{L}^{1 i} \xi_{R}^{k j}=\sum_{i, j=4}^{5} \epsilon_{123 i j} \alpha_{R}^{1 i}{ }^{T} C \xi_{R}^{k j}, \quad k=2,3 .
$$

Since the $\xi_{R}^{i j}$ fields pick up dynamical masses of order $\Lambda_{1}$ and then decouple, there are no technicolor-scale condensates of the form $\left\langle\sum_{i, j=4}^{5} \epsilon_{123 i j} \alpha_{R}^{1 i}{ }^{T} C \xi_{R}^{k j}\right\rangle$ with $k=2,3$. However, these terms can, in general, be induced by higher-order processes similar to those that can induce the $w_{i j, k \ell}$ shown in Eq. (4.24). In Fig. 6 we show graphs that can contribute to the Majorana bilinear ${\overline{\alpha^{c}}}_{L}^{13} w_{13,23} \xi_{R}^{23}=\alpha_{R}^{13}{ }^{T} C w_{13,23} \xi_{R}^{23}$ (and do so in the first sequence to be described below).

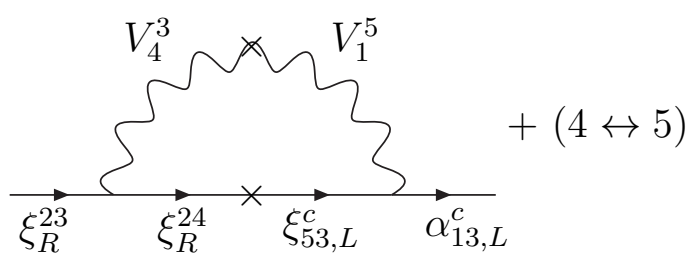

FIG. 6. Graphs that contribute to the Majorana bilinear $\alpha_{R}^{13}{ }^{T} C w_{13,23} \xi_{R}^{23}$ in symmetry-breaking sequence 1. 
To summarize, the full $39 \times 39$-dimensional neutrino mass matrix, M, factorizes into one diagonal block involving the $\mathrm{HC}$ singlet fields and another involving the $\mathrm{HC}$ non-singlets. The former can be seen to block-diagonalize further (implicit in the above discussion) into one block involving the TC-singlet fields and another involving the TC-non-singlets. The characteristic polynomial, $P(\lambda)$, associated with the diagonalization of $\mathrm{M}$ and the determination of its eigenvalues (the masses of the neutrino-like states), therefore factorizes into four parts, one of which, $P_{H C S-T C S}(\lambda)$, is associated with the HC-singlet and TC-singlet fields. The roots of the other three factors range in magnitude from $\Lambda_{1}$ down to $\Lambda_{T C}$. The factor $P_{H C S-T C S}(\lambda)$ contains two large roots of order $\Lambda_{1}$ and five other roots. These five roots will be of primary interest here since they contain three that form the mass eigenstates in the three electroweak-doublet neutrino interaction eigenstates, together with two that are primarily electroweak-singlet neutrinos. The detailed forms of these polynomial factors depend on the symmetry-breaking sequence and will be discussed below.

This general formalism can now be applied to any symmetry breaking sequence. We discuss two plausible sequences, depending on the relative strength of ETC and HC couplings.

\section{Symmetry-Breaking Sequence 1}

This sequence, leading to two further, distinct stages of ETC-symmetry breaking, is related to the one denoted $G_{a}$ in Ref. [6], but has further structure resulting from the additional $\omega_{p, R}^{\alpha}$ fermions not present there. In the effective theory below $\Lambda_{1}$, the ETC interaction, which is $\mathrm{SU}(4)_{E T C}$-symmetric, is again asymptotically free. As the energy decreases through a scale, $\Lambda_{2} \simeq 10^{2} \mathrm{TeV}$, the $\mathrm{SU}(4)_{E T C}$ and $\mathrm{SU}(2)_{H C}$ couplings become sufficiently strong to lead together to the condensation

$$
(4,2,1,1)_{0, R} \times(6,2,1,1)_{0, R} \rightarrow(\overline{4}, 1,1,1)
$$

with $\Delta C_{2}=5 / 2$ for $\mathrm{SU}(4)_{E T C}$ and $\Delta C_{2}=3 / 2$ for $\mathrm{SU}(2)_{H C}$. This breaks $\mathrm{SU}(4)_{E T C}$ to $\mathrm{SU}(3)_{E T C}$. The condensate is

$$
\begin{aligned}
& \left\langle\epsilon_{\alpha \beta} \epsilon_{12 j k \ell} \zeta_{R}^{1 j, \alpha T} C \zeta_{R}^{k \ell, \beta}\right\rangle= \\
& 2\left\langle\epsilon_{\alpha \beta}\left(\zeta_{R}^{13, \alpha T} C \zeta_{R}^{45, \beta}-\zeta_{R}^{14, \alpha T} C \zeta_{R}^{35, \beta}+\zeta_{R}^{15, \alpha T} C \zeta_{R}^{34, \beta}\right)\right\rangle
\end{aligned}
$$

and the twelve $\zeta_{R}^{i j, \alpha}$ fields in this condensate gain masses $\simeq \Lambda_{2}$. (To be precise, linear combinations of these fields form eigenstates with definite masses.)

The fact that the neutrino-like fields $\alpha_{R}^{1 i}$ transform as a 4 of SU(4) ETC, while the lefthanded neutrinos and technineutrinos transform as a $\overline{4}$, will lead to a strong suppression of 
relevant entries in the Dirac submatrix $M_{D}[3,22]$. This suppression depends only on the fact that the left- and right-handed components of the neutrinos transform according to relatively conjugate representations.

In the effective theory below $\Lambda_{2}$, both the $\mathrm{SU}(3)_{E T C}$ and the $\mathrm{SU}(2)_{H C}$ interactions are asymptotically free, so their couplings continue to increase as the energy scale decreases. At a scale $\Lambda_{3} \simeq 3 \mathrm{TeV}$, these interactions together are envisioned to lead to a condensation in the channel (where the numbers give the representations under $\mathrm{SU}(3)_{E T C} \times \mathrm{SU}(2)_{H C} \times G_{S M}$ )

$$
(3,2,1,1)_{0, R} \times(3,2,1,1)_{0, R} \rightarrow(\overline{3}, 1,1,1)_{0}
$$

with $\Delta C_{2}=4 / 3$ for $\mathrm{SU}(3)_{E T C}$ and $\Delta C_{2}=3 / 2$ for $\mathrm{SU}(2)_{H C}$. This breaks $\mathrm{SU}(3)_{E T C}$ to $\mathrm{SU}(2)_{T C}$. Without loss of generality, we may use the original $\mathrm{SU}(3)_{E T C}$ gauge symmetry to orient the condensate so that it takes the form

$$
\left\langle\epsilon_{123 j k} \epsilon_{\alpha \beta} \zeta_{R}^{2 j, \alpha T} C \zeta_{R}^{2 k, \beta}\right\rangle=2\left\langle\epsilon_{\alpha \beta} \zeta_{R}^{24, \alpha T} C \zeta_{R}^{25, \beta}\right\rangle
$$

In the low-energy effective field theory below $\Lambda_{3}$, the massless SM-singlet fermions then consist of $\zeta_{R}^{i j, \alpha}$ with $i j=12,23$, and $\omega_{p, R}^{\alpha}$ with $p=1,2$. In this energy interval the $\mathrm{SU}(2)_{H C}$ coupling continues to grow, as does the $\mathrm{SU}(2)_{T C}$ coupling. When the coupling $\alpha_{H C}$ becomes sufficiently strong, the hypercolor interaction can naturally produce (HC-singlet) condensates of the various remaining $\mathrm{HC}$-doublet fermions. In each case, $\Delta C_{2}=3 / 2$. Since the condensate (4.29) was formed via a combination of both $\mathrm{SU}(3)_{E T C}$ and $\mathrm{SU}(2)_{H C}$ interactions, while the present condensates are formed only by the $\mathrm{SU}(2)_{H C}$ interaction, and have the same value of $\Delta C_{2}$, it follows that the scale at which they form, denoted $\Lambda_{s}$ (where $s$ denotes $\mathrm{SU}(2)_{T C}$-singlet) satisfies $\Lambda_{s} \leq \Lambda_{3}$. There are six condensates of this type:

$$
\begin{gathered}
\left\langle\epsilon_{\alpha \beta} \zeta_{R}^{12, \alpha T} C \zeta_{R}^{23, \beta}\right\rangle \\
\left\langle\epsilon_{\alpha \beta} \zeta_{R}^{12, \alpha T} C \omega_{p, R}^{\beta}\right\rangle, \quad p=1,2 \\
\left\langle\epsilon_{\alpha \beta} \zeta_{R}^{23, \alpha T} C \omega_{p, R}^{\beta}\right\rangle, \quad p=1,2 \\
\left\langle\epsilon_{\alpha \beta} \omega_{1, R}^{\alpha T} C \omega_{2, R}^{\beta}\right\rangle .
\end{gathered}
$$

With these condensations, the 20 fields $\zeta^{i j, \alpha}$ and the four $\omega_{p, R}^{\alpha}$ fields condense out of the effective theory at energies below $\Lambda_{s}$, just as the six $\xi_{i j, R}$ condense out of the theory at energies below $\Lambda_{1}$. In what follows, shall take $\Lambda_{s} \simeq \Lambda_{3}$. This is reasonable, since the 
condensates (4.30) and (4.32) have the same symmetry behavior as the condensate (4.29) that forms at $\Lambda_{3}$, i.e., all three of these break $\mathrm{SU}(3)_{E T C}$ to $\mathrm{SU}(2)_{T C}$.

The condensates involving the $\omega_{p, R}^{\alpha}$ fields are important in the generation of the quark and lepton mass matrices. Without them, as we will describe shortly, a residual unbroken global symmetry would prevent the formation of some desirable matrix elements. The fact that the generational structure of the model is determined in this way by generation-dependent global symmetries is a feature of dynamical symmetry breaking, in which only gauge bosons are responsible for the communication of the breaking from the SM singlet fermion sector to the visible sector of the theory.

Finally, at the technicolor scale $\Lambda_{T C}$, the technifermions condense, breaking $\mathrm{SU}(2)_{L} \times$ $\mathrm{U}(1)_{Y}$ to $\mathrm{U}(1)_{\mathrm{em}}$. (Vacuum alignment arguments motivate color and electric charge conservation by the technicondensates [25]). The condition $\Lambda_{T C}<\Lambda_{s}$ is natural since, although both the $\mathrm{TC}$ and $\mathrm{HC}$ groups are $\mathrm{SU}(2)$, the leading coefficient for the beta function in the HC group below $\Lambda_{3}$ is larger than that for the TC group. Explicitly, with $\beta(\alpha)=-b_{0} \alpha^{2} / 2 \pi+\ldots$, we have $b_{0}=(11 / 3) N_{H C}-(2 / 3)(1 / 2) N_{h c d}=6$ for the $\mathrm{SU}(2)_{H C}$ theory and $b_{0}=(11 / 3) N_{T C}-(2 / 3)(1 / 2) N_{t c d}=2$ for the $\mathrm{SU}(2)_{T C}$ theory, since there are $N_{h c d}=4$ chiral SU$(2)_{H C^{-}}$doublet fermions $\left(\zeta_{R}^{12, \alpha}, \zeta_{R}^{23, \alpha}, \omega_{p, R}^{\alpha}\right)$ and $N_{t c d}=16$ chiral SU $(2)_{T C^{-}}$ doublet fermions active in this energy interval.

\section{E. Fermion Mass Matrices for Sequence 1}

The diagonal entries of the up-type quark mass matrix $M_{i j}^{(u)}$ are the conventional masses generated by the ETC mechanism, as given above in Eq. (2.9). The off-diagonal entries in $M_{i j}^{(u)}$ are generated by ETC gauge boson mixing of type (2.18). The general set of ETC gauge boson mixings generated by the SM-singlet condensates listed above is analyzed in the appendix. Here and throughout the text we use the results of this analysis. In the present case, the only ETC gauge boson mixing of type (2.18) that is generated is $V_{t}^{1} \leftrightarrow V_{t}^{3}$ with $t=4,5$. This gives rise to nonzero off-diagonal elements $M_{i j}^{(u)}$ only for $i j=13,31$ (which, in this approach, are equal in magnitude, as a consequence of (A17). Other off-diagonal entries will be shown to vanish from global symmetry considerations. The above ETC gauge boson mixing is described by a mass mixing function ${ }_{t}^{1} \Pi_{t}^{3}(0)$ with $t=4,5$, as described in the appendix.

The simplest graph contributing to the bilinear $\bar{u}_{a, 1, L} M_{13}^{(u)} u_{R}^{a, 3}$ is shown in Fig. 1, with $f=u, j=3, i=1$. The estimate is very similar to that of Eq. (2.9) with $i=3$, but suppressed by the ratio of the gauge boson mixing to the mass-squared of the heavier $\left(\Lambda_{1^{-}}\right.$ scale) ETC boson in the graph. With the TC theory walking up to the scale $\Lambda_{3}$, the integral 
will be dominated by momenta of that scale, and the gauge boson mixing estimate of Eq. (A9) may be used. We utilize the expression Eq. (2.7), with $a=1$, for the ETC gauge boson masses and thus estimate

$$
M_{13}^{(u)} \simeq \frac{\kappa \Lambda_{T C}^{2} \Lambda_{3}^{2}}{\Lambda_{1}^{2} \Lambda_{2}}
$$

where $\kappa \simeq 8 \pi / 3$. The result is the same order as the diagonal element $M_{11}^{(u)}$. The offdiagonal elements $M_{i j}^{(u)}$ with $i j$ different from 13 and 31 vanish identically, as a consequence of a residual global symmetry described below. With the ETC breaking scales of Eq. (2.10), this structure of $M_{i j}^{(u)}$ leaves the up-type quarks masses consistent with experiment.

For the down-type quarks and charged leptons, because we employ relatively conjugate ETC representations for the left- and right-handed components, all the elements of their mass matrices, diagonal and non-diagonal, will be suppressed, vanishing were it not for the ETC gauge boson mixing. The relevant non-diagonal ETC gauge boson 2-point functions are of the form $V_{i}^{4} \leftrightarrow V_{5}^{j}$ and $V_{i}^{5} \leftrightarrow V_{4}^{j}$ (see the appendix). The graphs that yield a down-type quark mass term in this model are given by the appropriate special cases of Fig. 2. We find that $M_{i j}^{(f)}$ for $f=d$, e has nonzero entries for all $(i j)$ except (12), (21), (23), and (32).

We estimate these entries, as above, by taking the theory to walk up to the lowest ETC scale $\Lambda_{3}$. We note again that the results will be given by the conventional ETC estimate of Eq. (2.9), but suppressed by the relevant ratios of mixings to gauge boson squared masses. Consider first the estimate of $M_{13}^{(d, e)}$. The appropriate gauge boson mixing term is given by Eq. (A8), and the full estimate is similar to that for $M_{13}^{(u)}$ :

$$
M_{13}^{(d, e)} \simeq \frac{\kappa \Lambda_{T C}^{2} \Lambda_{3}}{\Lambda_{1}^{2}}
$$

Turning next to the diagonal element $M_{22}^{(d, e)}$, we make use of the mixing term of Eq. (A11), which is suppressed relative to the previous mixings by $\Lambda_{3} / \Lambda_{2}$. We find

$$
M_{22}^{(d, e)} \simeq \frac{\kappa \Lambda_{T C}^{2} \Lambda_{3}^{4}}{\Lambda_{2}^{5}} .
$$

The two other diagonal entries are estimated in much the same way, making use of the gauge boson mixing terms of Eqs. (A15) and (A16). Recall that these involve iterations of previous mixings. The results are

$$
\begin{gathered}
M_{11}^{(d, e)} \simeq \frac{\kappa \Lambda_{T C}^{2} \Lambda_{3}^{3}}{\Lambda_{1}^{4}} \\
M_{33}^{(d, e)} \simeq \frac{\kappa \Lambda_{T C}^{2} \Lambda_{3}}{\Lambda_{1}^{2}} .
\end{gathered}
$$


There is mixing only between the first and third eigenstates. The resultant quark masses are $m_{s}=M_{22}^{(d)}$ and $m_{d} \sim m_{b} \simeq M_{13}^{(d)}$. With the $\Lambda_{i}$ choices of Eq. (2.10), these are $m_{s} \simeq 0.2$ $\mathrm{MeV}$ and $m_{d} \simeq m_{b} \simeq 1 \mathrm{MeV}$. Clearly, the down-type quark masses are suppressed far too much by this mechanism and, in addition, do not exhibit a full three-generation structure.

The diagonalization of $M^{(u)}$ and $M^{(d)}$ yields the quark mixing matrix $V$, according to (2.17). This mixing arises almost completely from the diagonalization of the down-quark mass matrix, so that $V=U_{L}^{(d) \dagger}$. Although the model does produce quark mixing, the $d$ - $b$ mixing angle is close to $\pi / 4$ and hence not phenomenologically acceptable. The same structural features apply to the charged lepton mass matrix. To leading order, the charged lepton masses are given by the same formulas as for the corresponding down-type quarks; however, color effects for the quarks will modify this equality.

For the neutrinos, sequence 1 yields nonzero entries in the Dirac submatrix $b_{i j}$ for $i j=$ $13,22,33$. A general relation in the present class of models is

$$
b_{i j}=M_{i j}^{(d, e)} \quad \text { for } \quad i=1,2,3 ; \quad j=2,3 .
$$

(Recall that there are only two right-handed, SM-singlet states below $\Lambda_{T C}$, and therefore there are no $j=1$ entries.) This equality is due to the fact that both the down-quark masses and the Dirac masses for the neutrinos are generated in the same way, employing relatively conjugate representations for the left- and right-handed components of these fields. Thus, Eq. 4.39) holds for any symmetry-breaking sequence in the present model. It a defect of the present class of models, since it means that these models have difficulty in simultaneously producing sufficiently large masses for down-type quarks and charged leptons on the one hand, and sufficiently small masses for neutrinos on the other hand.

Focusing on the neutrinos alone, the Dirac masses $b_{i j}$ could play a role in a seesaw mechanism, provided that a corresponding $2 \times 2$ Majorana mass matrix for the SM singlets (the $r_{i j}$ 's of Eq. (4.19)) is formed with the right magnitude. The 23 elements of this matrix are formed in sequence 1 via Fig. 4, but with magnitude much smaller than the Dirac terms. The reason for the smallness is that the fermions and gauge bosons of Fig. 4 have masses of order $\Lambda_{1}$, and the gauge boson mixing is soft above the smaller scale $\Lambda_{3}$. The relevant mixing, estimated in the appendix, arises from a combination of the mixings of Figs. 8 and 10. The only scale entering this combination is $\Lambda_{3}$, leading to the estimate

$$
r_{23} \simeq \frac{\kappa \Lambda_{3}^{6}}{\Lambda_{1}^{5}}
$$

For the above choices of $\Lambda_{1} \simeq 10^{3} \mathrm{TeV}$ and $\Lambda_{3} \simeq 4 \mathrm{TeV}, r_{23}$ is well below any of the nonzero $b_{i j}$ 's. This problem is alleviated as $\Lambda_{3} / \Lambda_{1}$ is increased, but since $\Lambda_{1}$ can be no smaller than 
a few hundred GeV to adequately suppress flavor-changing neutral current processes (see Section V), a realistic seesaw is not attainable for any $\Lambda_{3} \leq \Lambda_{1}$. This problem will be alleviated in sequence 2 , allowing a neutrino seesaw, but not at the same time generating realistic quark masses.

It is useful to exhibit the structure of the characteristic polynomial $P(\lambda)$ of $M$. Recall that $P(\lambda)$ factors in general into four parts, only one of which, $P_{H C S-T C S}(\lambda)$, has some roots smaller than $\Lambda_{T C}$. These occur as five of its seven roots (the other two are the masses of linear combinations of $\xi_{R}^{23}$ and $\xi_{R}^{45}$, of order $\left.\Lambda_{1}\right)$. $P_{H C S-T C S}(\lambda)$ takes the form

$$
P_{H C S-T C S}(\lambda)=\lambda P_{6}(\lambda)
$$

where the zero eigenvalue is the mass of $\nu_{1}$. Since the experimental data indicating neutrino oscillations only determines the differences of squares of neutrino masses $\left|\Delta m_{32}^{2}\right|$ and $\Delta m_{21}^{2}$, it allows the lightest eigenvalue to vanish. However, the model with this symmetry-breaking sequence is too simple to reproduce another feature from the data, namely the fact that $\nu_{1}$ mixes with $\nu_{2}$ and $\nu_{3}$ to form the interaction eigenstates. The factor $P_{6}(\lambda)$ is a polynomial of the form

$$
P_{6}(\lambda)=\sum_{j=0}^{6} p_{6, j} \lambda^{j}
$$

where for this sequence $p_{6,5}=p_{6,3}=p_{6,1}=0$, so that $P_{6}(\lambda)=P(-\lambda)$ and the roots come in opposite-sign pairs. The nonzero coefficients in Eq. (4.42) are

$$
\begin{gathered}
p_{6,4}=-\left(\Lambda_{1}^{2}+b_{13}^{2}+b_{22}^{2}+b_{33}^{2}+d_{1,45}^{2}+d_{3,45}^{2}+r_{23}^{2}+w_{13,23}^{2}\right) \\
p_{6,2}=\left(b_{13}^{2}+b_{22}^{2}+b_{33}^{2}+r_{23}^{2}\right) \Lambda_{1}^{2}-2 w_{13,23}\left(b_{13} d_{1,45}+b_{33} d_{3,45}\right) \Lambda_{1}+b_{22}^{2}\left(b_{13}^{2}+b_{33}^{2}\right) \\
+d_{1,45}^{2}\left(b_{22}^{2}+b_{33}^{2}+r_{23}^{2}\right)+d_{3,45}^{2}\left(b_{13}^{2}+b_{22}^{2}+r_{23}^{2}\right)+w_{13,23}^{2}\left(b_{22}^{2}+d_{1,45}^{2}+d_{3,45}^{2}\right) \\
-2 b_{13} b_{33} d_{1,45} d_{3,45}
\end{gathered}
$$

and

$$
\begin{aligned}
& p_{6,0}=-b_{22}^{2}\left[\left(b_{13}^{2}+b_{33}^{2}\right) \Lambda_{1}^{2}-2 w_{13,23}\left(b_{13} d_{1,45}+b_{33} d_{3,45}\right) \Lambda_{1}\right. \\
& \left.+b_{13}^{2} d_{3,45}^{2}+b_{33}^{2} d_{1,45}^{2}+w_{13,23}^{2}\left(d_{1,45}^{2}+d_{3,45}^{2}\right)-2 b_{13} b_{33} d_{1,45} d_{3,45}\right] .
\end{aligned}
$$

As noted above (see Fig. 5), while $d_{1,45}$ is nonzero in general, in order to get a nonzero $d_{i, 45}$ with $i=2,3$, one needs the ETC gauge boson mixing $V_{1}^{t} \rightarrow V_{i}^{t}$ with $i=2,3$ and 
$t=4,5$ to occur. In the present sequence, we have the mixing $V_{1}^{t} \rightarrow V_{3}^{t}$ and hence $d_{3,45}$ is also nonzero. Both $d_{1,45}$ and $d_{3,45}$ are estimated to be of order $\kappa \Lambda_{T C}^{2} \Lambda_{3} / \Lambda_{2}$, while $d_{2,45}$ and $d_{i, 23}$ for $i=1,2,3$ vanish. The graphs that yield $w_{13,23}$ in this sequence are shown in Fig. 6. This entry $w_{13,23}$ is estimated to be of order $\kappa \Lambda_{3}^{6} / \Lambda_{1}^{5}$. The other $w_{i j, k \ell}$ entries vanish. Because the $d_{1,45}, d_{3,45}$, and $w_{13,23}$ terms enter together with much larger terms in the various coefficients, they have a negligible effect on the eigenvalues. Dropping negligible terms, $P_{H C S-T C S}(\lambda)=\lambda\left(\lambda-\Lambda_{1}\right)\left(\lambda+\Lambda_{1}\right) P_{4}(\lambda)$, where $P_{4}(\lambda)$, evident from Eqs. (4.42)-(4.45), gives the nonzero eigenvalues of magnitude less than $\Lambda_{T C}$.

\section{F. Residual Generational Symmetries in Sequence 1}

The presence of zeros in the fermion mass matrices for sequence 1 is not due to the approximations made in the estimates. The zeros are exact, as a consequence of residual global generational symmetries. To see this, we first consider the model without the $\omega_{p, R}^{\alpha}$ fermions, in which case sequence 1 reduces to the sequence denoted $G_{a}$ in Ref. [6]. Then $M_{i j}^{(u)}$ is nonzero only for the diagonal entries $i j=11,22,33, M_{i j}^{(d)}$ is nonzero only for $i j=13,31,22$, the Dirac neutrino mass matrix is nonzero only for $i j=13,22$, and the $2 \times 2$ right-handed Majorana neutrino mass matrix vanishes identically.

The reason for the zeros is that all the elements of the above matrices, other than the diagonal elements of $M_{i j}^{(u)}$, require ETC gauge boson mixing, and the allowed mixings are in turn determined by global symmetries. The requirement of mixing for the Dirac matrices has already been explained. That mixing is necessary for the $2 \times 2$ right-handed Majorana neutrino mass matrix will be explained in the general discussion of the seesaw mechanism in Section IV H. The relevant global symmetry is a certain subgroup, $\mathrm{U}(1)_{f}$, of the generational global symmetry $U(3)_{f} \subset S U(5)_{E T C}$ acting on the first three ETC indices. It is generated by $e^{i \theta T_{f}}$ where $T_{f}$ is a combination of diagonal generators of $\mathrm{SU}(5)_{E T C}$ :

$$
T_{f}=\frac{1}{2} \operatorname{diag}(1,0,-1,0,0) \text {. }
$$

Although this $\mathrm{U}(1)_{f}$ is broken by the condensates in the $G_{a}$ sequence, the ETC gauge boson masses and mixings remain invariant under it. Since only the mixings $V_{4}^{2} \leftrightarrow V_{2}^{5}$ and $V_{4}^{1} \leftrightarrow V_{3}^{5}, V_{5}^{1} \leftrightarrow V_{3}^{4}$ respect this symmetry, only these are generated. To see that the ETC gauge boson masses and mixings are invariant under $\mathrm{U}(1)_{f}$, we first consider the condensate, (4.6), that forms at the highest scale $\Lambda_{1}$. It is not $\mathrm{U}(1)_{f}$-invariant, but the ETC gauge boson masses that emerge at this scale, arise via loop diagrams with internal fermion lines carrying the insertions of the dynamical mass terms corresponding to these condensates. Each involves only $\xi$ fields, but every loop of these fields requires an even number of insertions 
of the dynamical $\xi$ mass term. Each insert requires a corresponding insert with conjugate fields in order to close the loop, and this renders these diagrams invariant under the global $\mathrm{U}(1)_{f}$ transformation.

At the lower scales $\Lambda_{2}$ and $\Lambda_{3}$, it is the $\zeta$ fields that condense with each other, producing the lighter ETC gauge boson masses as well as the ETC gauge boson mixings. But one can see by inspection that all the condensates that form $\left(4.27,4.29\right.$, and 4.30) are $\mathrm{U}(1)_{f}$ invariant. Hence, so too are the ETC gauge boson masses and mixings.

The mixings allowed by the $\mathrm{U}(1)_{f}$ global symmetry determine, in turn, the allowed downquark, charged-lepton, and neutrino mass matrices. Only the diagonal up-type quark masses, the 22, 13 and 31 mass matrix entries for down-type quarks and charged leptons, and the 22 and 13 entries for the Dirac neutrino mass matrix can appear. The $\mathrm{U}(1)_{f}$ symmetry also forbids the appearance of any nonzero element of the $2 \times 2$ right-handed Majorana neutrino mass matrix $r_{i j}$. This symmetry can also be used to understand which $d_{i, j k}$ and $w_{i j, k \ell}$ entries are nonzero, but, as discussed above, the nonzero $d_{i, j k}$ and $w_{i j, k \ell}$ do not play an important role in determining the neutrino mass eigenvalues. .

The full theory with the $\omega_{p, R}^{\alpha}$ fermions produces the two condensates (4.31 and 4.32) which are formed with the $\zeta$ fields. These condensates are not invariant under $U(1)_{f}$. However, the loop diagrams responsible for the ETC gauge boson mixings require the insertion of an even number of dynamical masses corresponding to these additional condensates, half of which are complex conjugates of the others. Thus, the charge under $U(1)_{f}$ of such diagrams is always twice the charge of the single condensate involved, and therefore a residual discrete $\mathbb{Z}_{2}$ symmetry, generated by $e^{i \pi T_{f}}$, remains. This symmetry forbids the $12,21,23$, and 32 entries of quark and charged lepton mass matrices, and the 12, 23, and 32 entries of the Dirac neutrino mass matrix. But now the 13 and 31 entries of the up-type quarks, the 11 and 33 entries of the down-type quarks and charged leptons, and the 33 entry of the Dirac neutrino mass matrix are nonzero. This is a consequence of the fact that the mixings $V_{t}^{1} \leftrightarrow V_{t}^{3}$ with $t=4,5$ and $V_{4}^{1} \leftrightarrow V_{1}^{5}$ and $V_{4}^{3} \leftrightarrow V_{3}^{5}$ (plus the analogous mixings with the indices 4 and 5 interchanged) are generated, which lead, in turn, to the mixings $V_{i}^{4} \leftrightarrow V_{5}^{i}$ and $V_{i}^{5} \leftrightarrow V_{4}^{i}$ with $i=1$ and $i=3$.

Similarly, from the structure of the condensate at the scale $\Lambda_{1}$, in conjunction with the condensates at the lower scales $\Lambda_{2}$ and $\Lambda_{3}$, it follows that the entry $r_{23}=r_{32}$ of the (symmetric) $2 \times 2$ Majorana neutrino mass matrix is nonzero, while the diagonal elements $r_{22}$ and $r_{33}$ vanish. But as discussed above, these elements are far too small to drive the seesaw mechanism. Finally, the presence of the $\omega_{p, R}^{\alpha}$ fermions and their condensates allows a few more of the (less important) entries shown in Eqs. 4.21 and 4.24 to be non-vanishing. 


\section{G. Symmetry-Breaking Sequence 2}

We turn next to another symmetry breaking sequence which becomes plausible depending on the relative strengths of the ETC and HC couplings. It is related to the sequence denoted $G_{b}$ in Ref. [6], but has further structure resulting from the inclusion here of the ETC-singlet $\omega_{p, R}^{\alpha}$ fermions. Like sequence 1 , it does not lead to completely realistic masses and mixing angles, but it has some attractive features not present there.

At the first stage of breaking, $\Lambda_{1}$, the $\mathrm{SU}(5)_{E T C}$ breaks to $\mathrm{SU}(4)_{E T C}$ just as in sequence 1 , driven by condensation of the $\xi$ fields. As the energy decreases below $\Lambda_{1}$, the $\mathrm{SU}(4)_{E T C}$ and $\mathrm{SU}(2)_{H C}$ couplings increase, and now, at a scale $\Lambda_{B H C} \lesssim \Lambda_{1}(\mathrm{BHC}=$ broken HC), the $S U(4)_{E T C}$ interaction produces a condensation in the channel

$$
(6,2,1,1)_{0, R} \times(6,2,1,1)_{0, R} \rightarrow(1,3,1,1)_{0}
$$

With respect to $\mathrm{SU}(4)_{E T C}$, this channel has $\Delta C_{2}=5$ and is hence slightly more attractive than the initial condensation (4.6) with $\Delta C_{2}=24 / 5$ for the $\mathrm{SU}(5)_{E T C}$ interaction, but it can occur at the somewhat lower scale $\Lambda_{B H C}$ because it is repulsive with respect to hypercolor $\left(\Delta C_{2}=-1 / 4\right)$. This requires the $\mathrm{HC}$ coupling to be strong, but not so strong as to combine with the ETC interaction to produce the sequence 1 condensation Eq. (4.26).

With no loss of generality, one can orient the $\mathrm{SU}(2)_{H C}$ axes so that the condensate is

$$
\left\langle\epsilon_{1 i j k \ell} \zeta_{R}^{i j, 1 T} C \zeta_{R}^{k \ell, 2}\right\rangle+(1 \leftrightarrow 2)
$$

Since this is an adjoint representation of hypercolor, it breaks $\mathrm{SU}(2)_{H C} \rightarrow \mathrm{U}(1)_{H C}$. We let $\alpha=1,2$ correspond to the charges $Q_{H C}= \pm 1$ under the $\mathrm{U}(1)_{H C}$. This gives dynamical masses $\sim \Lambda_{B H C}$ to the twelve $\zeta_{R}^{i j, \alpha}$ fields involved.

At a lower scale, $\Lambda_{23}$, a combination of the $\mathrm{SU}(4)_{E T C}$ and $\mathrm{U}(1)_{H C}$ attractive interactions produces the condensation

$$
4 \times 4 \rightarrow 6
$$

with $\Delta C_{2}=5 / 4$ for the $\mathrm{SU}(4)_{E T C}$ interaction and associated condensate

$$
\left\langle\epsilon_{\alpha \beta} \zeta_{R}^{12, \alpha T} C \zeta_{R}^{13, \beta}\right\rangle
$$

The contraction with $\epsilon_{\alpha \beta}$ is included not to yield an $\mathrm{SU}(2)_{H C}$-invariant product, since this symmetry has already been broken, but instead to yield the requisite antisymmetric product $\mathrm{SU}(4)_{E T C}(4 \times 4)_{\text {antisym. }}=6$. The condensate $(4.50)$ is $\mathrm{U}(1)_{H C}$-invariant and breaks $\mathrm{SU}(4)_{E T C}$ to the direct product of $\mathrm{SU}(2)_{T C}$ and a local $\mathrm{U}(1)^{\prime}$ symmetry generated by 
$U(\theta)=e^{i \theta T_{d}}$, where $T_{d}$ is a certain linear combination of diagonal $\mathrm{SU}(5)_{E T C}$ generators, $T_{d}=(1 / 2) \operatorname{diag}(0,1,-1,0,0)$. (Here, we use the standard notation $\operatorname{diag}\left(a_{1}, \ldots, a_{n}\right)$ for the matrix with the given elements on the diagonal and other elements zero.)

The strong $\mathrm{U}(1)_{H C}$ interaction can also produce a number of additional condensates between fermions with opposite $\mathrm{U}(1)_{H C}$ charge (i.e. different values of the $\mathrm{HC}$ index $\alpha=$ $1,2)$. Since the $\mathrm{U}(1)_{H C}$ interaction is not asymptotically free, this should occur at the scale $\Lambda_{23}$. The condensates include eight involving $\zeta$ and $\omega$ fermions,

$$
\left\langle\zeta_{R}^{1 i, \alpha T} C \omega_{p, R}^{\beta}\right\rangle, \quad i=2,3 ; \quad p=1,2 ; \quad \alpha \neq \beta
$$

and four that involve only $\omega$ fields,

$$
\left\langle\omega_{p, R}^{\alpha T} C \omega_{p^{\prime}, R}^{\beta}\right\rangle, \quad 1 \leq p, p^{\prime} \leq 2 ; \alpha \neq \beta
$$

Each of the condensates (4.51) breaks the local $\mathrm{U}(1)^{\prime}$ symmetry, leaving a residual $\mathrm{SU}(2)_{T C} \times$ $\mathrm{U}(1)_{H C} \times G_{S M}$ continuous local symmetry.

Since this model involves only two ETC symmetry-breaking scales, $\Lambda_{1}$, and $\Lambda_{23}$, it does not obviously lead to a full hierarchy of three fermion generations. It is, nevertheless, of interest because of its different quark and lepton mixings and because it exhibits the mechanism proposed in Ref. [6] for the origin of light neutrino masses.

The technifermion condensation at the lower scale $\Lambda_{T C}$ is similar to that in sequence 1 , with the difference that since the technidoublet $\zeta_{R}^{1 i, \alpha}, i=4,5$ is not involved in any higher condensations, there are 18 rather than 16 chiral $\mathrm{SU}(2)_{T C}$ doublets present at $\Lambda_{T C}$, viz., $u_{\chi}^{i, a}$, $d_{\chi}^{i, a}, e_{\chi}^{i}$ for $\chi=L, R, n_{L}^{i}, \alpha_{R}^{1 i}$ and $\zeta_{R}^{1 i, \alpha}$ with $i=4,5, \alpha=1,2$, and one has the additional technicondensate

$$
\left\langle\epsilon_{123 j k} \epsilon_{\alpha \beta} \zeta_{R}^{1 j, \alpha T} C \zeta_{R}^{1 k, \beta}\right\rangle=2\left\langle\epsilon_{\alpha \beta} \zeta_{R}^{14, \alpha T} C \zeta_{R}^{15, \beta}\right\rangle
$$

Note that the presence of the $\epsilon_{\alpha \beta}$ is not due to the $\mathrm{SU}(2)_{H C}$ symmetry, which is broken, but rather represents the automatic antisymmetrization of the operator product in Eq. (4.53) on the indices $\alpha$ and $\beta$ due to the contraction with $\epsilon_{123 j k}$ and Fermi statistics.

The fact that the hypercolor-doublet technicolor-doublet fermions $\left(\zeta_{R}^{14, \alpha}, \zeta_{R}^{15, \alpha}\right)$ do not form bilinear technicondensates with hypercolor-singlet technicolor-doublet fermions follows from the exact $\mathrm{U}(1)_{H C}$ gauge symmetry. The presence of these two additional chiral technidoublets means that, when the technicolor theory is expressed in vector-like form (as it always can be, since $\mathrm{SU}(2)$ has only (pseudo)real representations), there are $N_{f}=9$ rather than $N_{f}=8$ vectorially coupled technifermions transforming according to the fundamental representation. Because of the strong-coupling nature of the TC theory, we consider that this 
fermion content is again consistent with the assumed existence of an approximate infrared fixed point in the confined phase, with associated walking behavior up to the lowest ETC scale $[12]$.

\section{H. Quark and Charged Lepton Mass Matrices for Sequence 2}

The fermion mass matrices for sequence 2 can be analyzed in the same way as for sequence 1. The zero and nonzero entries are summarized in Table II. The diagonal entries of the up-quark mass matrix are given by the general formula (2.9). But now, with only the two ETC-breaking scales $\Lambda_{1}$ and $\Lambda_{23}$, we have

$$
M_{11}^{(u)} \simeq \frac{\kappa \Lambda_{T C}^{2} \Lambda_{23}}{\Lambda_{1}^{2}}, \quad M_{22}^{(u)}=M_{33}^{(u)} \simeq \frac{\kappa \Lambda_{T C}^{2}}{\Lambda_{23}},
$$

where we recall $\kappa \simeq 8 \pi / 3$.

Off-diagonal elements of $M_{i j}^{(u)}$ are generated for the case $i j=23,32$, by the ETC gauge boson mixings of the form $V_{t}^{2} \leftrightarrow V_{t}^{3}$ with $t=4,5$ (see appendix). The scale associated with this mixing is $\Lambda_{23}$, and therefore the off-diagonal terms are of the same order as the $i j=22$ and 33 terms. The estimate is $M_{23}^{(u)}=M_{32}^{(u)}=\rho_{u} M_{22}^{(u)}$ with $\rho_{u} \simeq O(1)$. Thus, $M^{(u)}$ takes the form

$$
M^{(u)}=\frac{\kappa \Lambda_{T C}^{2}}{\Lambda_{23}}\left(\begin{array}{ccc}
\Lambda_{23}^{2} / \Lambda_{1}^{2} & 0 & 0 \\
0 & 1 & \rho_{u} \\
0 & \rho_{u} & 1
\end{array}\right) .
$$

For $\Lambda_{1}=10^{3} \mathrm{TeV}$, this gives $m_{u}=3 \mathrm{MeV}$ as in sequence 1 . For $m_{c}$ and $m_{t}$, the off-diagonal elements $M_{23}$ and $M_{32}$ play an important role. These masses are

$$
m_{t}, m_{c}=\frac{\kappa\left(1 \pm \rho_{u}\right) \Lambda_{T C}^{2}}{\Lambda_{23}}
$$

These are implicitly defined at the same momentum scale, say $m_{t}$. If we take $\Lambda_{23} \simeq 4 \mathrm{TeV}$, then $m_{t}$ can be fit as in sequence 1 . In order to fit $m_{c}$ one would need to have $\rho_{u}$ quite close to unity, namely $\rho_{u}=\left[1-\left(m_{c} / m_{t}\right)\right] /\left[1+\left(m_{c} / m_{t}\right)\right] \simeq 1-2\left(m_{c} / m_{t}\right)$, so some fine tuning would seem to be required. Still, it is interesting that sequence 2 can generate three mass scales for the up-type quark masses with only two ETC breaking scales, $\Lambda_{1}$ and $\Lambda_{23}$, as a result of the feature that the lower right-hand $2 \times 2$ submatrix of $M^{(u)}$ has all entries of comparable magnitude.

The mass matrices for the $Q=-1 / 3$ quarks and charged leptons arise solely via ETC gauge boson mixing. The requisite mixings, estimated in the appendix, are all suppressed by small mass ratios. We obtain 


$$
\begin{gathered}
M_{11}^{(d, e)} \simeq \frac{\kappa \Lambda_{T C}^{2} \Lambda_{23}^{4}}{\Lambda_{1}^{4} \Lambda_{B H C}} \\
M_{22}^{(d, e)}=M_{33}^{(d, e)} \simeq \frac{\kappa \Lambda_{T C}^{4}}{\Lambda_{23}^{3}},
\end{gathered}
$$

and

$$
M_{23}^{(d, e)}=M_{32}^{(d, e)} \simeq \frac{\kappa \Lambda_{T C}^{4}}{\Lambda_{23}^{3}},
$$

where, as in sequence 1, the overall numerical factor is simply taken from Eq. 2.9. Thus, $M^{(d, e)}$ has the form

$$
M^{(d, e)} \simeq \frac{\kappa \Lambda_{T C}^{4}}{\Lambda_{23}^{3}}\left(\begin{array}{ccc}
x & 0 & 0 \\
0 & 1 & \rho_{d} \\
0 & \rho_{d} & 1
\end{array}\right)
$$

where $\rho_{d} \simeq O(1)$ and

$$
x \simeq \frac{\Lambda_{23}^{7}}{\Lambda_{T C}^{2} \Lambda_{1}^{4} \Lambda_{B H C}} .
$$

With $\Lambda_{23} \simeq 4 \mathrm{TeV}, m_{\tau}$ is suppressed relative to $m_{t}$ by approximately $\Lambda_{T C}^{2} / \Lambda_{23}^{2}$, the right order of magnitude. The fact that the lower right-hand $2 \times 2$ sub-matrix of $M^{(d, e)}$ has all entries of comparable magnitude means that the correct value of $m_{\mu}$ could also emerge, but possibly involving some fine tuning. The same respective values obtain for $m_{s}$ and $m_{b}$. Recent lattice determinations of the current quark mass $m_{s}$ (i.e., the running mass evaluated at a scale well above $\Lambda_{Q C D}$, say at $2 \mathrm{GeV}$ ) yield values close to $m_{\mu}$ [16], the prediction of this model that $m_{s} \simeq m_{\mu}$ is a successful one. However, the corresponding prediction that $m_{b} \simeq m_{\tau}$ means that $m_{b}$ is too small by a factor of roughly $2-3$. The electron mass, $m_{e}$, and the down-quark mass, $m_{d}$, are given by Eq. (4.57), and are much too small.

For $f=u, d$, the matrices $M^{(f)}$ can be diagonalized by respective unitary transformations as in Eq. (2.15) with $U_{R}^{(f)}=U_{L}^{(f)}$. These unitary transformations have similar forms, and involve large mixing between the generation $i=2,3$ interaction eigenstates to form mass eigenstates in each charge subsector. Hence, in the product $V=U_{L}^{(u)} U_{L}^{(d) \dagger}$ which is the observed quark mixing matrix, one could obtain a result that agrees with the general properties of $V$ in the subsector of the two higher generations, namely that $V$ is largely diagonal, with small off-diagonal entries. These small off-diagonal entries reflect the slightly different structures of $M^{(u)}$ and $M^{(d)}$ and hence the slightly different forms of $U_{L}^{(u)}$ and $U^{(d)}$. However, the quark mixing is not realistic because the $i=1$ generation interaction eigenstate does not mix with the corresponding $i=2,3$ eigenstates. 


\section{Neutrino mass matrix for Sequence 2}

For sequence 2, the $b_{i j}$ Dirac neutrino matrix is identical to $M_{i j}^{(d, e)}$ for the relevant values of indices $i=1,2,3$ and $j=2,3$. Thus,

$$
\begin{gathered}
b_{12}=b_{13}=0 \\
b_{23}=b_{32} \simeq \frac{\kappa \Lambda_{T C}^{4}}{\Lambda_{23}^{3}} \\
b_{22}=b_{33} \simeq \frac{\kappa \Lambda_{T C}^{4}}{\Lambda_{23}^{3}} .
\end{gathered}
$$

For the $2 \times 2$ Majorana matrix, $r_{i j}$, we obtain $r_{22}=r_{33}=0$ and, from the evaluation of the graphs in Fig. 4,

$$
r_{23} \simeq \frac{\kappa \Lambda_{B H C}^{3} \Lambda_{23}^{3}}{\Lambda_{1}^{5}}
$$

As in the case of sequence 1 , the many inverse powers of $\Lambda_{1}$ emerge because the fermions and gauge bosons of Fig. 4 have masses of order $\Lambda_{1}$, and because the gauge boson mixing (described in the appendix) is soft above the scales $\Lambda_{B H C}$ and $\Lambda_{23}$.

The Majorana mass $r_{23}$ can be much larger with this sequence than in sequence 1 . The reason is the presence here of the scale $\Lambda_{B H C}$ which can be as large as $O\left(\Lambda_{1}\right)$. The ratio of the Dirac matrix elements and the right-handed Majorana elements is

$$
\frac{b_{23}}{r_{23}} \simeq \frac{\Lambda_{T C}^{4} \Lambda_{1}^{5}}{\Lambda_{23}^{6} \Lambda_{B H C}^{3}}
$$

This symmetry-breaking sequence can produce a successful seesaw mechanism of the type discussed in Ref. [6], yielding light neutrinos if one uses $\Lambda_{1} \simeq 10^{3} \mathrm{TeV}$ as above, $\Lambda_{B H C} \simeq$ $0.5 \Lambda_{1}$, and $\Lambda_{23} \simeq 10^{2} \mathrm{TeV}$. With these values, the ratio in Eq. (4.66) is $<<1$, the necessary condition for the seesaw of Ref. [6]. It can be seen that this is not possible in sequence 1 while maintaining a hierarchy among the ETC scales. However, even here the requisite value of $\Lambda_{23}$ is too large to produce a sufficiently heavy top quark, so that the model does not appear capable of explaining both light neutrino masses and the top quark mass.

We discuss the neutrinos further, supposing that $\Lambda_{23}$ is allowed to be much larger than $4 \mathrm{TeV}$ as above. For this purpose, it is sufficient [6] to consider the $5 \times 5$ submatrix $M_{0}$ of $M_{H C S}$ that involves only $\left(\nu_{e}^{c}, \nu_{\mu}^{c}, \nu_{\tau}^{c}, \alpha^{12}, \alpha^{13}\right)_{R}$. Other fermions have gained larger masses. Diagonalizing $M_{0}$, we find that the model yields the following neutrino masses. Here the 
eigenvalue corresponding to $m\left(\nu_{3}\right)$ is negative, so we absorb this minus sign in an appropriate redefinition of the neutrino fields.

$$
\begin{aligned}
& m\left(\nu_{3}\right) \simeq \frac{\left(b_{23}+b_{22}\right)^{2}}{r_{23}}+O\left(\frac{b_{i j}^{4}}{r_{23}^{3}}\right) \simeq \frac{\kappa(1+y)^{2} \Lambda_{T C}^{8} \Lambda_{1}^{5}}{\Lambda_{23}^{9} \Lambda_{B H C}^{3}} \\
& m\left(\nu_{2}\right) \simeq \frac{\left(b_{23}-b_{22}\right)^{2}}{r_{23}}+O\left(\frac{b_{i j}^{4}}{r_{23}^{3}}\right) \simeq \frac{\kappa(1-y)^{2} \Lambda_{T C}^{8} \Lambda_{1}^{5}}{\Lambda_{23}^{9} \Lambda_{B H C}^{3}}
\end{aligned}
$$

where $y=b_{22} / b_{23} \simeq O(1)$, and

$$
m\left(\nu_{e}\right)=m\left(\nu_{1}\right)=0
$$

Since $y$ is positive and $O(1)$, sequence 2 gives the normal hierarchy $m\left(\nu_{3}\right)>m\left(\nu_{2}\right)$. Thus, one can extract the value of $m\left(\nu_{3}\right)$ from the measured value of $\left|\Delta m_{32}^{2}\right|[26,27]$, namely $m\left(\nu_{3}\right) \simeq \sqrt{\left|\Delta m_{32}^{2}\right|} \simeq 0.05 \mathrm{eV}$, and $m\left(\nu_{2}\right)$ from the measured value of $\Delta m_{21}^{2}$ [28,29], namely $m\left(\nu_{2}\right) \simeq \sqrt{\Delta m_{21}^{2}} \simeq 0.008 \mathrm{eV}$. With $\Lambda_{1}=10^{3} \mathrm{TeV}, \Lambda_{B H C}=0.5 \Lambda_{1}$, and $\Lambda_{23}=10^{2} \mathrm{TeV}$, these experimental values are fit with the choice $y \simeq 0.4$.

The non-vanishing Dirac masses, $b_{i j}$, are then $O(10-100) \mathrm{KeV}$ and the right-handed Majorana masses, $r_{23}$, are or order $O\left(10^{2}\right) \mathrm{GeV}$. The two large eigenvalues of $M_{0}$ are

$$
\lambda_{h_{1}}=r_{23}+\frac{\left(b_{23}+b_{22}\right)^{2}}{r_{23}}+O\left(\frac{b_{i j}^{4}}{r_{23}^{3}}\right)
$$

and

$$
\lambda_{h_{2}}=-r_{23}-\frac{\left(b_{23}-b_{22}\right)^{2}}{r_{23}}+O\left(\frac{b_{i j}^{4}}{r_{23}^{3}}\right) .
$$

To leading order, the resultant mass eigenstates involve maximal mixing (i.e., with an angle of $\pm \pi / 4)$ of $\left|\alpha_{R}^{12}\right\rangle$ and $\left|\alpha_{R}^{13}\right\rangle$. We label the corresponding neutrino-like states as $\left|\nu_{h_{j}}\right\rangle$ with masses $m\left(\nu_{h_{1}}\right)=\lambda_{h_{1}}$ and, after appropriate rephasing, $m\left(\nu_{h_{2}}\right)=-\lambda_{h_{2}}$.

Experimentally, there is evidence for large lepton mixing [26]- [30]. Because of the dominant off-diagonal structure of $r_{i j}$ and the fact that $b_{i j}$ has a lower $2 \times 2$ submatrix with comparable-size entries for $i j=22,23,32,33$, there is naturally large mixing between the second and third generations of neutrino mass eigenstates to form the interaction eigenstates $\nu_{\mu}$ and $\nu_{\tau}$. Indeed, the $2 \times 2$ submatrix in $U_{\nu}$ (the matrix that diagonalizes the full neutrino mass matrix), acting on $\left(\nu_{\mu}, \nu_{\tau}\right)$ is the rotation $R\left(\theta_{\nu, 23}\right)$ with $\theta_{\nu, 23}=\pi / 4$, up to small corrections. The observed lepton mixing matrix defined by Eq. (4.10) involves the product of the relevant terms from $U_{\nu}$ with those from the matrix $U_{L}^{(e)}$ that is involved in 
the diagonalization of the charged lepton mass matrix. Since the analogous $2 \times 2$ submatrix of $U^{(e)}$ acting on the $(\mu, \tau)$ subsector is also of the form $R\left(\theta_{e, 23}\right)$ with $\theta_{e, 23}=-\pi / 4$ up to small corrections (owing to the relation $M_{22}^{(e)}=M_{33}^{(e)}$ ), these maximal mixings cancel, and the resultant observed lepton mixing matrix $U$ with this sequence does not exhibit maximal mixing.

In summary, the model with the symmetry-breaking sequence 2 can exhibit the mechanism proposed in Ref. [6] for explaining light neutrino masses in theories with dynamical electroweak symmetry breaking. The sequence has the defect that $m_{e}$ and $m_{d}$ are too small, and it appears difficult to obtain satisfactory simultaneous fits to $m_{t}$ (and $m_{\tau}$ ) and to the neutrino masses.

We note again that the characteristic polynomial, $P(\lambda)$, of the full $39 \times 39$ mass matrix contains 34 roots of magnitude $\Lambda_{T C}$ or larger. The remaining five roots, of primary interest, are those for $m\left(\nu_{i}\right), i=1,2,3$ and for $m\left(\nu_{h_{j}}\right)$ discussed above. These, together with two roots approximately equal to $\pm \Lambda_{1}$, occur as the roots of the factor $P_{H C S-T C S}(\lambda)$ for the seven HC-singlet, TC-singlet fermions. For the present sequence,

$$
P_{H C S-T C S}(\lambda)=\lambda\left(\lambda^{2}-\Lambda_{1}^{2}-d_{1,45}^{2}\right) P_{4}(\lambda)
$$

where

$$
\begin{aligned}
& P_{4}(\lambda)=\lambda^{4}-\left(b_{22}^{2}+b_{23}^{2}+b_{32}^{2}+b_{33}^{2}+r_{23}^{2}\right) \lambda^{2} \\
& -2 r_{23}\left(b_{22} b_{23}+b_{32} b_{33}\right) \lambda+\left(b_{22} b_{33}-b_{23} b_{32}\right)^{2} .
\end{aligned}
$$

As was true for sequence 1 , one sees that $d_{1,45}$ has a negligible effect on the eigenvalues, since

it enters in the combination $\Lambda_{1}^{2}+d_{1,45}^{2}$ with the much larger quantity $\Lambda_{1}^{2}$. Other $d_{i, j k}$ 's and $w_{i j, k \ell}$ terms are zero.

\section{J. Residual Generational Symmetries in Sequence 2}

As with sequence 1, we can understand the zeros in the various mass matrices from the point of view of residual global generational symmetries and selection rules governing the breaking of these symmetries by the condensates. Again, we first consider the truncated model without the $\omega_{p, R}^{\alpha}$ fields. In this case, the condensates involving the $\zeta$ fields are invariant under a local $\mathrm{U}(1)_{f}$ symmetry generated by $e^{i \theta(x) T_{f}^{\prime}}$ where $T_{f}^{\prime}$ is a different combination of diagonal generators of $\mathrm{SU}(5)_{E T C}$ :

$$
T_{f}^{\prime}=\frac{1}{2} \operatorname{diag}(0,1,-1,0,0) .
$$


Proceeding to the full model with the $\omega_{p, R}^{\alpha}$ fields, one can apply the discussion for sequence 1 with obvious changes to conclude that $M^{(u)}=0$ for $i j=12,21,13,31 ; M^{(d, e)}=0$ for

$i j=12,21,13,31 ; b_{i j}=0$ for $i j=12,13$; and $r_{22}=r_{33}=0$. Similar reasoning can be applied to the $d_{i, j k}$ and $w_{i j, k \ell}$ to conclude that among these only $d_{1,45}$ is nonzero.

There is a general relation connecting sequences 1 and 2 in insofar as they involve condensates of the $\zeta$ fields and resultant ETC gauge boson mixings, namely that these condensates and mixings in sequence 2 are related to those for sequence 1 by the interchange of the ETC indices 1 and 2 (holding other ETC indices fixed) with appropriate changes in the condensation scale. This is because, before the inclusion of the $\omega_{p, R}^{\alpha}$ fields, the generators of the $\mathrm{U}(1)_{f}$ symmetries in each case are related by precisely this interchange of the ETC indices. This interchange also relates the respective $\zeta$ condensates in sequences 1 and 2 . Thus, the condensate (4.27) at scale $\Lambda_{2}$ in sequence 1 goes to the condensate (4.48) at scale $\Lambda_{B H C}$ in sequence 2 ; the condensate (4.29) at $\Lambda_{3}$ in sequence 1 goes to the condensate (4.53) at $\Lambda_{T C}$ in sequence 2 ; the condensate (4.30) at $\Lambda_{3}$ in sequence 1 goes to (4.50) at $\Lambda_{23}$ in sequence 2 ; the condensate (4.31) at $\Lambda_{3}$ in sequence 1 goes to the same condensate, now at $\Lambda_{23}$, in sequence 2 ; and the condensate (4.32) at $\Lambda_{3}$ in sequence 1 goes to the $i=3$ case of (4.51) at $\Lambda_{23}$ in sequence 2 . This relation between sequences 1 and 2 is evident in the summary tables I and II.

\section{PHENOMENOLOGY}

While the explicit model presented here is not fully realistic, it does have several realistic features such as natural intra-family mass splittings and non-trivial mixing angles. These features suggest that some ingredients in the general class of models we have described could appear in a successful theory of fermion masses and mixing. In this section we discuss some generic experimental constraints and phenomenological properties of this class of models.

\section{A. Precision Electroweak Constraints}

A natural first step in theories of dynamical electroweak symmetry breaking is to focus on corrections to the $W$ and $Z$ propagators, in particular the $S$ and $T$ parameters [31,32]. Since the class of models considered here produces large intra-generational mass splittings, it is important to evaluate the contributions to $\Delta \rho=\alpha_{e m} T$ from ETC interactions. These contributions arise because these ETC interactions (unlike TC) do not respect a custodial, $\mathrm{SU}(2)_{R}$ symmetry and lead, for example, to $m_{t}>>m_{b}$, as a consequence of the representation assignments $(2.11)$ and (2.12). The dominant contribution to $\Delta \rho$ may be estimated 
by concentrating on the lowest ETC scale, where $m_{t}$ and $m_{b}$ are generated. There will be, generically, contributions to $\Delta \rho$ arising from corrections to the basic technicolor mass generation mechanism for the $W$ and $Z$, due to the emission and reabsorption of single ETC gauge bosons of this lightest mass scale.

These contributions to $\Delta \rho$ can be roughly estimated by recalling that the momentum scale of the technicolor mass generation mechanism is set by $\Lambda_{T C} \simeq 300 \mathrm{GeV}$, and the emission and reabsorption of an ETC gauge boson will lead to a denominator factor of $1 / \Lambda_{l}^{2}$, where $\Lambda_{l}$ is the lightest ETC scale $\left(=\Lambda_{3}\right.$ in sequence 1 and $\Lambda_{23}$ in sequence 2$)$. Note that since the momentum integrals here are rapidly damped at scales above $\Lambda_{T C}$, there can be no walking enhancement as in the case of the fermions mass estimates (2.9). Drawing on the discussion leading to Eq. (2.7) (with $a \simeq O(1)$ ) and noting that the factors of $g_{E T C}$ cancel in $\Delta \rho$, we estimate

$$
\Delta \rho_{E T C} \simeq \frac{2 b \Lambda_{T C}^{2}}{3 \Lambda_{l}^{2}},
$$

where $b$ is a factor of order unity reflecting the relative strength of custodial symmetry breaking at the lowest ETC scale. With $\Lambda_{T C} \simeq 300 \mathrm{Gev}$ and $\Lambda_{l} \simeq 4 \mathrm{Tev}$, this gives $\Delta \rho \simeq\left(3.8 \times 10^{-3}\right) b$ or equivalently, $T \simeq 0.48 b$. In comparing this estimate with experiment, we first caution that with the inclusion of the high-statistics, high-precision data from the $\mathrm{NuTeV}$ experiment at Fermilab, studies have found that attempts at global fits to precision electroweak data using only the corrections represented by the parameters $S$ and $T$ give poor values of $\chi^{2}$ per degree of freedom [11]. In the absence of a satisfactory fit with the $S$ and $T$ parameters, it is difficult to draw a firm conclusion regarding the comparison of the estimate (5.1) with this data. Constraints obtained from global fits using pre-NuTeV are summarized in (Fig. 10.3) of Ref. [32]. The allowed elliptical regions in the $S, T$ plane are plotted for three illustrative values of SM Higgs mass, 115, 300, and $1000 \mathrm{GeV}$. Choosing the $10^{3} \mathrm{GeV}$ value to correspond most closely to a technicolor theory, one observes that the ellipse has a central value of about $(S, T) \simeq(-0.1,0.3)$ and a $1 \sigma$ upper boundary that extends up to about 0.6 in $T$ and, in a correlated manner, about 0.1 in $S$. Our estimate above of the (E)TC contribution to $T$ of about 0.5 is consistent with this bound if $b \sim 1$, and would lie closer to the central value for $b \lesssim 1$, which is possible, given the theoretical uncertainties in the strong-coupling estimate.

Roughly speaking, the $S$ parameter is sensitive to all new physics at the electroweak/technicolor scale. The smallness of $S$ is an indication, subject to the uncertainties associated with the strong technicolor interactions, that the number of degrees of freedom there is small. That was one of the reasons that we chose to use the technicolor group $\mathrm{SU}(2)_{T C}$. The evaluation of $S$ is difficult in a strongly coupled theory such as (extended) 
technicolor. Having chosen the effective Higgs mass of $1000 \mathrm{GeV}$ in order to compare with the fit to data in Ref. [32], we have already, in effect, included some strong TC contributions. In addition to these strong TC contributions, one must also include the effect of SM-nonsinglet (pseudo)-Nambu-Goldstone bosons. It was noted in Ref. [10], that for a theory with walking, that is, with an approximate IR conformal fixed point, the TC contribution to $S$ is naturally reduced. This observation is based on an application of the Weinberg spectral function sum rules and includes the effects of both technifermions and (pseudo)-Nambu-Goldstone bosons. It is possible that this would lead to an $S$ parameter in agreement with current experimental limits. We assume here that this is the case.

Another quantity to check is the ETC correction to the $Z b \bar{b}$ vertex and hence to the comparison of the measured value $R_{b}=\Gamma(Z \rightarrow b \bar{b}) / \Gamma(Z \rightarrow$ hadrons $)=0.21664 \pm 0.00068$ with the SM prediction $R_{b}=0.21569 \pm 0.00016$ (from the global fit given in the current PDG listings [32]). There are two main contributions to the corrections to this vertex, from graphs in which the $Z$ produces (i) a virtual techniquark $D \bar{D}$ pair which exchange a $V_{3}^{t}$, $t=4,5$, becoming a $b \bar{b}$ pair, and (ii) a virtual $b \bar{b}$ pair which exchange the ETC gauge boson corresponding to the lightest diagonal $\mathrm{SU}(5)_{E T C}$ generator coupling to the third generation, viz., $T_{15}=\operatorname{diag}(0,0,-2,1,1)$ in our notation. The contributions (i) and (ii) were studied for a conventional ETC theory in Ref. [33] and were found to enter with opposite sign and hence to tend to cancel each other. We find that the same is true in our ETC model with conjugate representations. Because of the opposite-sign nature of these ETC contributions and in view of the theoretical uncertainty in the calculation due to the strong ETC coupling, we conclude that the ETC correction to $R_{b}$ can be consistent with the experimentally measured value of $R_{b}$ and its comparison with the SM prediction.

\section{B. Flavor-Changing Neutral Processes}

In early studies of extended technicolor, it was sensibly assumed that the ETC theory would generate flavor-changing neutral currents (FCNC), and it was argued that these led to severe constraints on the models. The measured rates for processes such as $K^{0}-\bar{K}^{0}$ mixing, $K_{L} \rightarrow \mu^{+} \mu^{-}$, and the upper limits on the branching ratios for decays such as $K^{+} \rightarrow \pi^{+} \mu^{ \pm} e^{\mp}$, $K_{L} \rightarrow \mu^{ \pm} e^{\mp}$, and $\mu \rightarrow e \gamma$ led to the conclusion that the ETC scales had to be very high. However, since nothing approaching a realistic ETC theory was written down, the assumed

mixing was put in by hand, typically into a set of four-fermion couplings. Since in the present paper we have constructed a model in which quark mixing is actually generated by the dynamics, we can re-examine the question of FCNC operators. Although the model is not fully realistic, it can perhaps provide some new insight into this issue. 


$$
\text { 1. } K^{+} \rightarrow \pi^{+} \mu^{ \pm} e^{\mp}
$$

We first review the situation in a conventional ETC model in which the quarks and charged leptons of both chiralities transform according to the same representation of $G_{E T C}$. We focus on $K^{+} \rightarrow \pi^{+} \mu^{ \pm} e^{\mp}$ decays because it is these for which recent experimental limits have been obtained (the charge-conjugate decay modes $K^{-} \rightarrow \pi^{-} \mu^{\mp} e^{ \pm}$are, up to small CPviolating effects, equivalent). At the quark level, the decay $K^{+} \rightarrow \pi^{+} \mu^{ \pm} e^{\mp}$ corresponds to the process $\bar{s} \rightarrow \bar{d} \mu^{ \pm} e^{\mp}$ with a spectator $u$ quark. At tree level, only one of these transitions occurs, namely $\bar{s} \rightarrow \bar{d} \mu^{+} e^{-}$; this arises via a diagram in which an $\bar{s}$ antiquark emits a virtual $V_{2}^{1}$ ETC gauge boson, thereby transforming into a $\bar{d}$ antiquark, and the $V_{2}^{1}$ ETC gauge boson produces the pair $\mu^{+} e^{-}$. (For the $\bar{s} \rightarrow \bar{d} \mu^{-} e^{+}$process one would need the ETC gauge boson mixing $V_{2}^{1} \leftrightarrow V_{1}^{2}$.) From the lowest-order graph, one obtains the quark-level amplitude

$$
A m p\left(\bar{s} \rightarrow \bar{d} \mu^{+} e^{-}\right) \simeq\left(\frac{g_{E T C}}{\sqrt{2}}\right)^{2}\left[\bar{s} \gamma_{\lambda} d\right] \frac{1}{M_{1}^{2}}\left[\bar{e} \gamma^{\lambda} \mu\right]
$$

Since $g_{E T C} \simeq O(1)$ at the scale $M_{1}$, this is only an approximate estimate of the amplitude. Normalizing to $K_{\mu 3}^{+}$decay and taking into account that $\left\langle\pi^{+}\left|U_{+}\right| K^{+}\right\rangle=\sqrt{2}\left\langle\pi^{0}\left|V_{-}\right| K^{+}\right\rangle$, where $T_{ \pm}, U_{ \pm}$, and $V_{ \pm}$denote the usual flavor-SU(3) shift operators, we have

$$
\frac{\Gamma\left(K^{+} \rightarrow \pi^{+} \mu^{+} e^{-}\right)}{\Gamma\left(K^{+} \rightarrow \pi^{0} \mu^{+} \nu_{\mu}\right)}=\frac{16}{\left|V_{u s}\right|^{2}}\left(\frac{g_{E T C}}{g_{2}}\right)^{4}\left(\frac{m_{W}}{M_{1}}\right)^{4}
$$

where $g_{E T C}$ denotes the ETC gauge coupling at the scale $\Lambda_{1}$, and $g_{2}$ denotes the weak $\operatorname{SU}(2)_{L}$ gauge coupling (at the scale $m_{W}$, given by $g_{2}=e / \sin \theta_{W}=0.65$ ). With $\left|V_{u s}\right|=0.22$, this yields the lower limit

$$
M_{1} \gtrsim\left(1.6 \times 10^{3} \mathrm{TeV}\right)\left(\frac{g_{E T C}}{2 \pi}\right)\left[\frac{10^{-12}}{B\left(K^{+} \rightarrow \pi^{+} \mu^{+} e^{-}\right)}\right]^{1 / 4} .
$$

The current upper limit is $B R\left(K^{+} \rightarrow \pi^{+} \mu^{+} e^{-}\right)<2.9 \times 10^{-11}$ [38] from the Yale-BNL experiment E865. Using the published limit and the relation $M_{i}=\left(g_{E T C} / 4\right) a \Lambda_{i}$, we get the rough lower bound

$$
\Lambda_{1} \gtrsim \frac{1}{a} O(450) \quad \mathrm{TeV}
$$

where $a$ is expected to be $O(1)$.

The calculation is different in the class of models considered in this paper, because the left- and right-handed down-type quarks transform according to conjugate representations of $G_{E T C}$. In turn, the structure of the amplitudes is different for models of DEC and DES 
type. We focus here on the DEC-type model discussed in the text. At the quark level the process $\bar{s} \rightarrow \bar{d} \mu^{+} e^{-}$arises from two contributions (at the leading, tree level): (i) a $\bar{s}_{L}$ makes a transition to the $\bar{d}_{L}$ and emits a virtual $V_{2}^{1}$, which can only couple (without ETC gauge boson mixing) to the current $\bar{e}_{R} \gamma^{\lambda} \mu_{R}$, and (ii) a $\bar{s}_{R}$ makes a transition to $\bar{d}_{R}$, emitting a $V_{1}^{2}$, which couples to the current $\bar{e}_{L} \gamma^{\lambda} \mu_{L}$. Hence, the leading contributions to the amplitude at the quark level is

$$
A m p\left(\bar{s} \rightarrow \bar{d} \mu^{+} e^{-}\right)=\frac{g_{E T C}^{2}}{2 M_{1}^{2}}\left[\left[\bar{s}_{L} \gamma_{\lambda} d_{L}\right]\left[\bar{e}_{R} \gamma^{\lambda} \mu_{R}\right]+\left[\bar{s}_{R} \gamma_{\lambda} d_{R}\right]\left[\bar{e}_{L} \gamma^{\lambda} \mu_{L}\right]\right]
$$

This should be contrasted with Eq. (5.2) for a conventional ETC model. Another difference is the fact that, whereas $\bar{s}$ does not go to $\bar{d} \mu^{-} e^{+}$at tree level in a conventional ETC model, it does in the present class of models, in particular, a DEC-type model. One finds

$$
A m p\left(\bar{s} \rightarrow \bar{d} \mu^{-} e^{+}\right)=\frac{g_{E T C}^{2}}{2 M_{1}^{2}}\left[\left[\bar{s}_{L} \gamma_{\lambda} d_{L}\right]\left[\bar{\mu}_{L} \gamma^{\lambda} e_{L}\right]+\left[\bar{s}_{R} \gamma_{\lambda} d_{R}\right]\left[\bar{\mu}_{R} \gamma^{\lambda} e_{R}\right]\right]
$$

One obtains a lower limit on $M_{1}$ and hence $\Lambda_{1}$ that is comparable with the limits given above for conventional ETC models. Our choice $\Lambda_{1}=10^{3} \mathrm{TeV}$ is consistent with these lower bounds.

$$
\text { 2. } K_{L}^{0} \rightarrow \mu^{ \pm} e^{\mp}
$$

In conventional ETC models, the tree-level contribution to the decay $K_{L}^{0} \rightarrow \mu^{ \pm} e^{\mp}$ vanishes because the amplitude picks out the axial-vector part of the hadronic current, $\left[\bar{s} \gamma^{\lambda}\left(c_{V}-c_{A} \gamma_{5}\right) d\right]$, but this current is vectorial in these conventional models. However, this is no longer the case in the type of model considered here, so one does get a tree-level contribution to this amplitude, mediated by the $V_{2}^{1}$ and $V_{1}^{2}$ ETC gauge bosons. The amplitudes for $K_{L} \rightarrow \mu^{ \pm} e^{\mp}$ can be obtained in a straightforward manner from the quark amplitudes given above in Eq. (5.6) and (5.7). The current upper bound on the branching ratio for this decay, from an experiment at BNL, is [39] $B R\left(K_{L} \rightarrow \mu^{+} e^{-}\right)+B R\left(K_{L} \rightarrow \mu^{-} e^{+}\right)<4.7 \times 10^{-12}$. Our choice of $\Lambda_{1}=10^{3} \mathrm{TeV}$ is consistent with this limit.

\section{Neutral Meson Systems}

Because of the transitions $M^{0} \leftrightarrow \bar{M}^{0}$, where $M=K, B_{d}, B_{s}$, or $D$, the mass eigenstates of these neutral non-self-conjugate mesons involve linear combinations of $\left|M^{0}\right\rangle$ and $\left|\bar{M}^{0}\right\rangle$ with mass differences between the respective heavier $(h)$ and lighter $(\ell)$ eigenstates 
$m_{M_{h}^{0}}-m_{M_{\ell}^{0}} \equiv \Delta m_{M}$ given by $2 \operatorname{Re}\left(\left\langle\bar{M}^{0}\left|\mathcal{H}_{e f f}\right| M^{0}\right\rangle\right)$ for the kaon system, where $K_{L}$ and $K_{S}$ have quite different lifetimes, and by $2\left|\left\langle\bar{M}^{0}\left|\mathcal{H}_{\text {eff }}\right| M^{0}\right\rangle\right|$ for the $B$ and $D$ systems, where the heavier and lighter states have essentially the same lifetimes. The smallness of the $K_{L}-K_{S}$ mass difference provided early evidence that the weak neutral current should be diagonal. Experimentally [32]:

$$
\begin{aligned}
\Delta m_{K} & =(0.530 \pm 0.001) \times 10^{10} \mathrm{~s}^{-1}=(3.49 \pm 0.006) \times 10^{-12} \mathrm{MeV} \\
\Delta m_{B_{d}} & =(0.489 \pm 0.008) \times 10^{12} \mathrm{~s}^{-1}=(3.27 \pm 0.05) \times 10^{-10} \mathrm{MeV} \\
\Delta m_{B_{s}} & >13 \times 10^{12} \mathrm{~s}^{-1}=0.89 \times 10^{-8} \mathrm{MeV} \quad(95 \% \mathrm{CL}) \\
\Delta m_{D} & <7 \times 10^{10} \mathrm{~s}^{-1}=0.5 \times 10^{-10} \mathrm{MeV} \quad(95 \% \mathrm{CL}) .
\end{aligned}
$$

The standard model accounts for the two measured mass differences and agrees with the limits on the other two [32,34]. This thereby places constraints on non-SM contributions such as those from ETC gauge boson exchanges.

Early studies of constraints on conventional ETC theories, in which the SM fermions of both chiralities transform according to the same ETC representations, from $K^{0}-\bar{K}^{0}$ mixing amplitude assumed an ETC contribution of strength $g_{E T C}^{2} /\left(8 M_{E T C}^{2}\right) \simeq 1 / \Lambda_{E T C}^{2}$, as would arise if an $d \bar{s}$ pair would annihilate to form a virtual ETC gauge boson in the $s$-channel, which would then produce a $s \bar{d}$ pair, and similarly for $t$-channel ETC gauge boson exchange. Using this assumption, these studies obtained lower bounds of order $\Lambda_{E T C} \gtrsim 10^{3} \mathrm{TeV}$. However, the virtual $V_{2}^{1}$ ETC gauge boson produced by the $d \bar{s}$ pair cannot produce a $s \bar{d}$ pair in these theories; rather, one must have the mixing $V_{2}^{1} \leftrightarrow V_{1}^{2}$ to do this. (This type of ETC gauge boson mixing is also necessary for box diagram contributions involving exchange of two ETC gauge bosons.) This ETC gauge boson mixing suppresses the transition substantially, by a factor of order ${ }_{1}^{2} \Pi_{2}^{1}(0) / \Lambda_{1}^{2}<<1$ (see appendix). Hence, the lower bound on $\Lambda_{1}$ from $K^{0}-\bar{K}^{0}$ mixing in conventional ETC theories is less stringent than $10^{3} \mathrm{TeV}$. (As discussed above, a bound of this order does hold in these conventional ETC theories, but it is due to the experimental upper bound on $B R\left(K^{+} \rightarrow \pi^{+} \mu^{+} e^{-}\right)$.) The same comment about the necessity of ETC gauge boson mixing applies for the other $M^{0}-\bar{M}^{0}$ transitions.

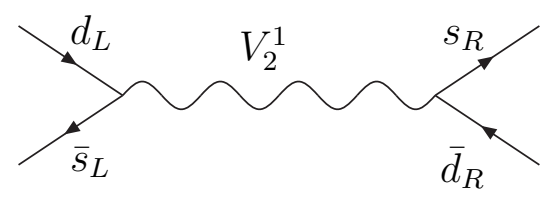


FIG. 7. Graph that contributes in the $s$-channel to $K^{0}-\bar{K}^{0}$ transition amplitude in the present type of ETC theory, where the left- and right-handed components of the $Q=-1 / 3$ quarks transform according to conjugate representations of $G_{E T C}$. The analogous graph with chiralities $L$ and $R$ interchanged and $V_{2}^{1}$ replaced by $V_{1}^{2}$ also contributes to this transition. These do not require any $V_{2}^{1} \leftrightarrow V_{1}^{2}$ ETC gauge boson mixing. The corresponding $t$-channel graphs also contribute.

The situation concerning these transitions is different in our class of ETC models. Here there are diagrams that can contribute at tree level, without any ETC gauge boson mixing. These are the same for both DEC and DES-type models. An example for $K^{0}-\bar{K}^{0}$ mixing is shown in Fig. 7. One finds

$$
\Delta m_{K} \simeq 2 \frac{\left(g_{E T C} / \sqrt{2}\right)^{2}}{M_{1}^{2}} \operatorname{Re}\left(\left\langle\bar{K}^{0}\left|\left[\bar{s}_{L} \gamma_{\lambda} d_{L}\right]\left[\bar{s}_{R} \gamma^{\lambda} d_{R}\right]\right| K^{0}\right\rangle\right) .
$$

A similar formula applies for $B_{d}^{0}-\bar{B}_{d}^{0}$ mixing, with the change noted above and the replacement $s \rightarrow b$, and similarly for $B_{s}^{0}-\bar{B}_{s}^{0}$ mixing, with the replacements $s \rightarrow b, d \rightarrow s$, and $M_{1} \rightarrow M_{2}$. These estimates involve theoretical uncertainty owing to the strong ETC coupling and the fact that the hadronic matrix elements are not measured and must be estimated, e.g. by lattice gauge theory simulations; in addition, there is a question of the degree of short-distance dominance for the $K^{0}-\bar{K}^{0}$ amplitude. We find that with the values of $\Lambda_{1} \sim 1000 \mathrm{TeV}$ used above, our model predicts values of $\Delta m_{K}$ and $\Delta m_{B_{d}}$ larger than the measured values. For example, using values of $f_{B}$ and hadronic matrix elements from lattice measurements [35], we estimate $\Delta m_{B_{d}} \simeq 8 \times 10^{-10} \mathrm{MeV}$. This is a potential problem for the class of ETC models considered in this paper. It can be ameliorated if the model has somewhat larger values of $\Lambda_{1}$. In addition, with our choice of $\Lambda_{2} \sim 50 \mathrm{TeV}$, we find $\Delta m_{B_{s}} \sim 3 \times 10^{-7} \mathrm{MeV}$, a value well above the SM prediction. The measurement of this quantity will clearly be very important for the class of models considered here.

Our predictions could be reduced also in models that have the property of walking not only from $\Lambda_{T C}$ to the lowest ETC scale, but to higher energy scales such as was assumed in Ref. [6]. This could make possible higher ETC breaking scales and thereby suppress these flavor-changing neutral current effects while maintaining reasonable agreement with experimental values of SM fermion masses.

By contrast, the contribution of our model to $D^{0}-\bar{D}^{0}$ mixing is quite small, because of the necessity of ETC gauge boson mixing (since the up-type quarks $u_{L}$ and $u_{R}$ are in the same ETC representations). The main graphs contributing to this transition include an $s$-channel diagram in which the $c \bar{u}$ pair annihilates to form a $V_{1}^{2}$ gauge boson, which must go through a ${ }_{2}^{1} \Pi_{1}^{2}(0)$ to yield a $V_{2}^{1}$ which then produces the final-state $u \bar{c}$ pair, and the corresponding $t$-channel graph. This yields a resulting value of $\Delta m_{D}$ which is negligibly small compared with the current upper limit on this mass difference. 
We remind the reader that we are not considering CP-violating phases in this paper. Furthermore, in the specific model examined in this paper, with either symmetry breaking sequence, there is not sufficient generatonal mixing to generate CKM CP violation. Assuming that the class of models being considered can lead to realistic mixing and CP violation (both dynamical in origin), there is good reason to expect that the $\mathrm{CP}$ violation associated with non-standard-model physics will be suppressed by mixing effects among both the fermions and the ETC gauge bosons. If this is the case, then the ETC scales we have used will not lead to unacceptable levels of $\mathrm{CP}$ violation in the neutral kaon system.

Other processes can be analyzed similarly. For example, the contribution to $b \rightarrow s \gamma$ is safely smaller than the value of this process inferred from experiment because of the necessity of ETC gauge boson mixing, which results because the relevant operator $\propto\left[\bar{s} \sigma_{\mu \nu} b\right] F^{\mu \nu}$ flips chirality (where $F^{\mu \nu}$ denotes the electromagnetic field strength tensor).

\section{Non-diagonal Neutrino Neutral Currents}

If the leptons of a given charge and chirality have different weak $T$ and $T_{3}$, then the neutral leptonic current is non-diagonal in terms of mass eigenstates [37]. The class of models considered here includes SM-singlet neutrino-like states that form Dirac mass terms with the left-handed neutrinos. It is a convention whether one writes these as right- or lefthanded; if one writes them as left-handed and applies the criteria of Ref. [37], it follows that the neutrino part of the weak neutral current is non-diagonal in terms of mass eigenstates. This non-diagonality is a generic feature of this class of models. The coefficients of terms that are non-diagonal in mass eigenstates are $\simeq b_{i j} / r_{23}$ and hence are extremely small in a realistic model that incorporates the seesaw mechanism of Ref. [6] to yield light neutrinos.

\section{Intermediate-Mass Dominantly Electroweak-Singlet Neutrinos}

\section{General}

Our mechanism [6] for getting light neutrinos generically leads to neutral leptons with masses that are intermediate between the higher ETC scales and the mass scales characterizing the primary mass eigenstates in the three observed electroweak-doublet neutrinos. As linear combinations of interaction eigenstates, they are composed almost completely of SM-singlet fields, with only a small admixture of electroweak-doublet neutrinos. They have masses of order $O\left(10^{-1}-10^{2}\right) \mathrm{GeV}$, depending on the type of symmetry-breaking sequence. The lower end of the mass range for these intermediate-mass neutrinos is illustrated by the 
model of Ref. [6], while the rest of the range is illustrated by sequence 2. (The absence of light neutrino mass eigenstates that are primarily electroweak-singlets, often called (light) "sterile" neutrinos, is in good agreement with indications from neutrino oscillation experiments [26-29]).

The mass eigenstates $\left(\nu_{1}, \nu_{2}, \nu_{3}, \nu_{h_{1}}, \nu_{h_{2}}\right)$ mix to form the interaction eigenstates $\nu_{\ell}$ with $\ell=e, \mu, \tau$ and the SM-singlets $\alpha^{12}$ and $\alpha^{13}$. Writing out Eq. (4.9) explicitly (dropping the $m$ subscript on the mass eigenstates), we thus have (in a basis where the charged lepton mixing matrix is diagonal)

$$
\left|\nu_{\ell}\right\rangle=\sum_{i=1}^{3} U_{\ell i}\left|\nu_{i}\right\rangle+U_{\ell h_{1}}\left|\nu_{h_{1}}\right\rangle+U_{\ell h_{2}}\left|\nu_{h_{2}}\right\rangle
$$

where $U_{e j} \equiv U_{1 j}, U_{\mu j} \equiv U_{2 j}$, etc. The small admixture coefficients $U_{\ell h_{1}}$ and $U_{\ell h_{2}}$ are of order the ratio of the Dirac to right-handed Majorana mass entries $b_{i j} / r_{23}$, for the relevant (dominant) coefficients. In our model, the diagonalization of the full neutrino mass matrix leads to the eigenstates $\left|\nu_{h_{j}}\right\rangle$ with $j=1,2$; these mass eigenstates contain small admixtures of electroweak-doublet neutrinos, with coefficients of order $b_{i j} / r_{23}$. As discussed in [6], and exhibited also in our present model with symmetry-breaking sequence 2 , a dominant $b_{i j}$ element has a typical size given by $b_{23} \simeq M_{23}^{(d)}$, and the relevant entry in $r_{i j}$ is displayed in Eq. (4.65). The ratio $b_{23} / r_{23}$ is given by Eq. (4.66) and has a value of order $10^{-7}$. Hence, generically, one expects that the coefficients $\left|U_{\ell j}\right|$ would be of this size.

\section{Massive Neutrino Emission Via Mixing in Weak Decays}

At the lower end of the mass range for the intermediate-mass neutrinos, there are some interesting and testable experimental consequences: the emission, via lepton mixing, of heavy neutrinos in weak decays, as constrained by the available phase space. Tests for such heavy neutrino emission via lepton mixing have been proposed, and data analyzed to set bounds [40]. These tests were carried out in a number of experiments on $\pi^{+} \rightarrow \mu^{+} \nu_{\mu}, \pi^{+} \rightarrow e^{+} \nu_{e}$, $K^{+} \rightarrow \mu^{+} \nu_{\mu}$ and $K^{+} \rightarrow e^{+} \nu_{e}$ decays. A decay of this type would consist of one decay of a given charged pseudoscalar meson $\pi^{+}$or $K^{+}$into the set of light neutrinos, which propagate effectively coherently and recoil against an outgoing charged lepton, and another decay yielding the heavy neutrino mass eigenstate(s) $\left|\nu_{h_{j}}\right\rangle$ with $j=1,2$, also recoiling against the outgoing charged lepton. The signature of the heavy neutrino emission would thus be a peak in the charged lepton momentum spectrum at an anomalously low value. Since one can calculate the branching ratio for the emission of the massive neutrino as a function of the mixing parameter and neutrino mass, an upper limit on the branching ratio can be converted 
into an upper limit on $\left|U_{\ell h_{j}}\right|$ for this assumed neutrino mass. Experimental searches in $\pi_{e 2}^{+}$, $K_{\mu 2}^{+}$, and $K_{e 2}^{+}$decays have yielded upper limits of order $\left|U_{\ell h_{j}}\right|^{2} \lesssim 10^{-7}$ for $m\left(\nu_{h_{j}}\right)$ in the interval 100-300 MeV [32], for $\ell=e, \mu$. For the type of model considered here, with heavy neutrinos having masses above the phase space limit, this constraint is clearly satisfied. Even if the heavy neutrinos were as light as a few hundred $\mathrm{MeV}$, as in the type of model of Ref. [6], one would expect that this squared mixing matrix element would be of order $10^{-(10 \pm 2)}$, with the theoretical uncertainty in the exponent indicated. Hence, the model of Ref. [6] is in accord with these limits.

These limits could be improved drawing on more recent experiments. Data on the decay $K^{+} \rightarrow \mu^{+} \nu_{\mu}$ that was recorded as an auxiliary part of the very high-statistics BNL experiments E787 and E949 that observed $K^{+} \rightarrow \pi^{+} \nu \bar{\nu}$ decay [41] could be used to search for the emission of a heavy neutrino down to a branching ratio sensitivity that is estimated to be of order $10^{-9}$ and perhaps better [42].

At the extreme low end of the range of expected masses of the intermediate-scale neutrino, it could also be emitted in muon decay, e.g., as $\mu^{+} \rightarrow \bar{\nu}_{h_{j}} e^{+} \nu_{e}$. These decays into heavy neutrinos would be suppressed, relative to the usual decay into light neutrinos, $\mu^{+} \rightarrow \bar{\nu}_{\mu} e^{+} \nu_{e}$, by the respective leptonic mixing matrix factors $\left|U_{\mu h_{j}}\right|^{2}$ and $\left|U_{e h_{j}}\right|^{2}$, in addition to reduced phase space. The observed $e^{+}$spectrum would be due to all of the kinematically allowed decays. In turn, this would lead to modifications of the observed $\mu$ decay spectral parameters $\rho, \eta, \xi$, and $\delta$. One can use the agreement of these parameters with the standard model values to set upper bounds on heavy neutrino emission [43]. Again, these bounds allow the current type of intermediate-mass neutrino.

\section{Non-orthogonality of Observed $\left|\nu_{e}\right\rangle$ and $\left|\nu_{\mu}\right\rangle$}

Here we mention an effect that is expected to be quite small but is of conceptual interest. The abstract Hilbert space states $\left|\nu_{e}\right\rangle$ and $\left|\nu_{\mu}\right\rangle$ are ortho-normal. However, the experimental definition of the states $\left|\nu_{e}\right\rangle$ and $\left|\nu_{\mu}\right\rangle$ is as the neutrinos accompanying the emission of $e^{+}$and $\mu^{+}$in the pseudoscalar decays $\mathrm{Ps}^{+} \rightarrow e^{+} \nu_{e}$ and $\mathrm{Ps}^{+} \rightarrow \mu^{+} \nu_{\mu}$ and their conjugates, where $\mathrm{Ps}^{+}$denotes $K^{+}$or $\pi^{+}$. It is via these sources that standard accelerator neutrino beams are formed [44]. In a theory with heavy neutrinos, these neutrino states, as experimentally defined, are not, in general, orthogonal [24,37]. This follows from the fact that in the linear combinations (5.13), the states with sufficiently large masses are kinematically forbidden

from occurring in the above particle or nuclear decays. For example, if $\nu_{h_{1}}$ and $\nu_{h_{j}}$ are sufficiently heavy that they do not occur in $\pi^{+} \rightarrow \ell^{+} \nu_{\ell}$ or $K^{+} \rightarrow \ell^{+} \nu_{\ell}$ decays, then

$$
\left\langle\nu_{e} \mid \nu_{\mu}\right\rangle_{\text {exp. }}=U_{1 h_{1}}^{*} U_{2 h_{1}}+U_{1 h_{2}}^{*} U_{2 h_{2}}
$$




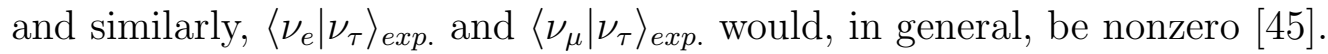

$$
\text { 4. } \Delta L=2 \text { Decays }
$$

Because the physical intermediate-mass neutrinos are Majorana states, there are contributions to $|\Delta L|=2$ decays such as neutrinoless double beta decay of nuclei and the particle decays $K^{+} \rightarrow \pi^{-} \ell^{+} \ell^{\prime+}$, where $\ell, \ell^{\prime}=e e, e \mu, \mu \mu$. Searches for neutrinoless double beta decay of nuclei yield an upper limit, the form of which depends on whether the mass of the virtual neutrino in the propagator is large or small compared with the Fermi momentum, $\sim 200 \mathrm{MeV}$, of the nucleons in the nucleus. The primary mass eigenstates $\nu_{i}, i=1,2,3$, entering into $\nu_{e}$ produce an amplitude involving $\left\langle m_{\nu}\right\rangle=\left|\sum_{i=1}^{3} U_{e i}^{2} m\left(\nu_{i}\right)\right|$. In the present model, the intermediate-mass neutrinos $\nu_{h_{j}}, j=1,2$, will, in general, also contribute to this amplitude, through the couplings $U_{e h_{j}}, j=1,2$. If the masses $m\left(\nu_{h_{j}}\right)$ are near the lower end of the expected range, then the resultant $\left\langle m_{\nu}\right\rangle=\left|\sum_{i=1}^{3} U_{e i}^{2} m\left(\nu_{i}\right)+\sum_{j=1,2} U_{e h_{j}}^{2} m\left(\nu_{h_{j}}\right)\right|$. Model-dependent calculations of the relevant nuclear matrix elements are employed in order to extract an upper limit on $\left\langle m_{\nu}\right\rangle$. Current searches yield limits of $\left\langle m_{\nu}\right\rangle \lesssim O(1) \mathrm{eV}$ [46]. At present there are a number of efforts to perform new experiments to search for neutrinoless double beta decay with sensitivities considerably below this limit in $\left\langle m_{\nu}\right\rangle$ (e.g., [46]). The mechanism for light neutrinos in Ref. [6] provides a further motivation for these searches. This can be seen as follows: if $m\left(\nu_{h_{j}}\right)$ for $j=1,2$ are near the lower end of the expected range, say of order $100 \mathrm{MeV}$ and $\left|U_{e h_{j}}\right|$ is as large as $10^{-5}$, we estimate the resultant $\left\langle m_{\nu}\right\rangle \simeq 10^{-2} \mathrm{eV}$. While this is in accord with current limits, it might yield a signal in future searches. However, with walking only up to the lowest ETC scale, $\Lambda_{3}$ or $\Lambda_{23}$ as in the present paper, $m\left(\nu_{h_{j}}\right.$ are much heavier. This alters the form of the neutrino propagator and leads to a negligible contribution by these intermediate-mass neutrinos to the amplitude. Moreover, since our model predicts a normal hierarchy $m\left(\nu_{3}\right)>m\left(\nu_{2}\right)>m\left(\nu_{1}\right)$ and since $m\left(\nu_{3}\right)$ enters in the amplitude for neutrinoless double beta decay suppressed by the very small mixing matrix factor $u_{e 3}^{2}$, one does not expect the primary mass eigenstates $\nu_{i}, i=1,2,3$ to produce this type of decay at a significant level.

Another possible manifestation of the violation of total lepton number is in $|\Delta L|=2$ particle decays such as $K^{+} \rightarrow \pi^{-} \mu^{+} \mu^{+}$; searches for these were discussed in Refs. [47,38]. The current limit is [38] $B R\left(K^{+} \rightarrow \pi^{-} \mu^{+} \mu^{+}\right)<3.0 \times 10^{-9}$. The intermediate-mass neutrinos $\nu_{h_{j}}, j=1,2$ would contribute to this decay. However, in the present model this contribution is expected to be negligible compared to the current limit. 


\section{Astrophysical and Cosmological Constraints}

There are also astrophysical and cosmological constraints on a heavy unstable neutrino with a mass in the range of order $0.1 \mathrm{GeV}$ to $100 \mathrm{GeV}$. To discuss these, one has to calculate the lifetime of the neutrino. The decay amplitude involves two types of contributions. First, there are charged-current contributions arising from graphs in which the $\nu_{h_{j}}$, via its admixture in $\nu_{\ell}$, for $\ell=e$ and $\mu$, emits a virtual $W^{+}$, producing an outgoing $\ell$, with the $W^{+}$ then producing an $e^{+} \nu_{e}$. In the case of $\ell=\mu$, this decay would be suppressed by small phase space if $m\left(\nu_{h_{j}}\right)$ were only slightly greater than $m_{\mu}$ and would be forbidden if $m\left(\nu_{h_{j}}\right)<m_{\mu}$. Second, there are contributions arising from neutral-current processes since, in this type of model with electroweak-singlet neutrinos the neutral current is non-diagonal [37]. These decays are dominantly of the form $\nu_{h_{j}} \rightarrow \nu_{i} \bar{\nu}_{\ell} \nu_{\ell}$, where the $\nu_{i}$ and $\nu_{\ell}$ refer to the light neutrino mass eigenstates. The contribution of each decay involves a small coefficient reflecting Eq. (5.13). From the charged-current contributions we have

$$
\Gamma_{\nu_{h_{j}}}=\tau_{\nu_{h_{j}}}^{-1}=\left(\frac{m\left(\nu_{h_{j}}\right)}{m_{\mu}}\right)^{5}\left(\sum_{\ell}\left|U_{\ell h_{j}}\right|^{2}\right) \Gamma_{\mu}
$$

where $\tau_{\mu}=\Gamma_{\mu}^{-1}=2 \times 10^{6} \mathrm{sec}$. is the muon lifetime. There are also contributions from the non-diagonal neutral-current couplings. Combining these, we estimate, as an example, that for $m\left(\nu_{h_{j}}\right) \simeq 100 \mathrm{GeV}, \tau_{\nu_{h_{1}}} \simeq \tau_{\nu_{h_{2}}} \simeq 10^{-7}$ sec. Owing to the fifth power dependence, $\Gamma_{\nu_{h_{j}}}$ varies rapidly as a function of $m\left(\nu_{h_{j}}\right)$, and approaches existing limits when $m\left(\nu_{h_{j}}\right)$ decreases to $\mathrm{O}(100) \mathrm{MeV}$.

Since the intermediate-mass neutrino decays on a short time scale, it is, of course, not subject to the mass limits for neutrinos that are stable on the time scale of the age of the universe and thus affect the mean mass density of the universe. The relevant astrophysical and cosmological constraints include those from (i) primordial (big-bang) nucleosynthesis and (ii) large-scale structure formation. A recent analysis of these constraints is given in [48]. A perusal of these constraints suggests that a neutrino with the mass of order hundreds of $\mathrm{MeV}$ to hundreds of $\mathrm{GeV}$, and the corresponding lifetime given by (5.15) is allowed.

\section{Dark Matter Candidates}

Models incorporating the mechanism of Ref. [6] for light neutrinos can yield interesting candidates for dark matter. Although the model studied in this paper is not fully realistic, we believe that it does yield some generic predictions for possible dark matter candidates that are of interest. 
A first important point concerns a state which might appear to be an appealing dark matter candidate in technicolor theories, namely the technibaryon composed of technineutrinos. In our model, this would be the particle state created by the action of the adjoint of the operator

$$
\frac{1}{\sqrt{2}} \epsilon_{123 j k} \bar{n}_{L}^{j} \alpha_{R}^{1 k}
$$

on the vacuum. This has a mass $\simeq 2 \Lambda_{T C} \simeq O(600) \mathrm{GeV}$. If one neglects ETC gauge boson mixing, one might infer that this state is stable. However, in our model with either symmetry-breaking pattern, the particle (5.16) decays via a process in which the $\alpha^{15}$, say, makes a transition to $\alpha^{12}$, emitting a virtual ETC gauge boson $V_{2}^{5}$ which, via mixing, goes to $V_{4}^{2}$; the $V_{4}^{2}$ is absorbed by the $\bar{n}^{4}$, going to $\bar{n}^{2}=\bar{\nu}_{\mu}$ (where mass eigenstates are understood for the actual final-state particles). This decay is kinematically allowed since the final state has a mass $m\left(\nu_{h_{j}}\right)$, which is less than the mass of the initial state. (The $\alpha^{12}$, or more precisely, the lighter of the $\nu_{h_{j}}$, decays, e.g. to $e^{-} e^{+} \nu_{e}$.)

We next display dark matter candidates for symmetry-breaking sequences 1 and 2 . For sequence 1 , we recall that at a scale $\Lambda_{s}$, which we take to be $\simeq \Lambda_{3}$, the hypercolor-invariant condensates (4.30)-(4.33) form, due to the strong hypercolor interaction . Consequently, linear combinations of the states $\zeta_{R}^{12, \alpha}, \zeta_{R}^{23, \alpha}$, and $\omega_{p, R}^{\alpha}$ with $p=1,2$ form mass eigenstates, which may be denoted $s_{q, R}^{\alpha}$ with $1 \leq q \leq 4$, in order of increasing mass. The $\mathrm{SU}(2)_{H C}$-singlet bound state of the lightest two of these, formed by the action of the adjoint of the operator

$$
\frac{1}{\sqrt{2}} \epsilon_{\alpha \beta} s_{1 R}^{\alpha T} C s_{2 R}^{\beta}
$$

on the vacuum, is stable. The stability can be understood as a consequence of the fact that this particle is the lightest hypercolor baryon (a boson, since $N_{H C}$ is even) and $\mathrm{SU}(2)_{H C}$ is an exact symmetry. Hence, the corresponding particle, with a mass $\simeq 2 \Lambda_{3} \simeq 8 \mathrm{TeV}$, might provide a dark matter candidate. Since this technibaryon is composed of SM-singlet fermions, its interactions with regular matter would be highly suppressed, since they would proceed dominantly via exchange of ETC gauge bosons, whose masses are considerably larger than the electroweak scale. It would thus be a very weakly coupled WIMP.

With symmetry-breaking sequence 2 , the candidate(s) for dark matter arise(s) differently. We recall that the fermions $\zeta_{R}^{12, \alpha}, \zeta_{R}^{13, \alpha}$, and $\omega_{p, R}^{\alpha}$ form condensates at the scale $\Lambda_{23}$, as given by Eqs. (4.50), (4.51), and (4.52), with the difference that these are only invariant under $\mathrm{U}(1)_{H C}$, since $\mathrm{SU}(2)_{H C}$ has been broken at the higher scale $\Lambda_{B H C}$. Linear combinations of these fermions thus gain dynamical masses $\sim \Lambda_{23}$. However, the ETC-nonsinglets among these fermions can decay. For example, a $\zeta_{R}^{12, \alpha}$ can make a transition to the $\zeta^{14, \alpha}$, a member 
of a lighter mass eigenstate, emitting a virtual $V_{4}^{2}$ ETC gauge boson which then produces fermion-antifermion pairs such as $u^{5, a} \bar{u}_{2, a}=U^{5, a} \bar{c}_{a}(a=$ color index $)$, etc. This decay is kinematically allowed since the initial fermion is part of a mass eigenstate with mass $\sim \Lambda_{23}$, while the illustrative final state given above has mass $\sim 2 \Lambda_{T C}$, and similarly for other final states. The same comment applies to $\zeta_{R}^{13, \alpha}$. The $\omega_{p, R}^{\alpha}$ 's with $p=1,2$, which are part of mass eigenstates with masses of order $2 \Lambda_{23} \simeq 8 \mathrm{TeV}$, do not decay in this manner, but can annihilate as $\omega_{p, R}^{+}+\omega_{q, R}^{-} \rightarrow \geq n \gamma_{H C}$ where $n \geq 2$, we have used our labelling of the $\mathrm{U}(1)_{H C}$ charge + and - for $\alpha=1,2$, respectively, and $\gamma_{H C}$ denotes the gauge boson corresponding to the $\mathrm{U}(1)_{H C}$ gauge symmetry. If there is an initial asymmetry in the $\mathrm{U}(1)_{H C}$ charge in the universe, this is preserved, and the resultant lighter linear combination of $\omega_{p, R}^{ \pm}, p=1,2$ could serve as a dark matter candidate.

The model with sequence 2 has another dark matter candidate. To show this, we recall that in this sequence the fermions $\zeta_{R}^{1 j, \alpha}$, where $j=4,5$, transforming as TC doublets remain light down to the $\Lambda_{T C}$ scale (in contrast to sequence 1, where they gain dynamical masses $\sim$ $\Lambda_{2}$ ). Because of the exact $\mathrm{SU}(2)_{T C}$ symmetry, these fermions do not mix with technisinglets, and because of the residual exact $\mathrm{U}(1)_{H C}$ gauge symmetry, they also do not mix with HCsinglets. The condensate (4.53) has the consequence that the mass eigenstates are $t_{1,2, R}^{\alpha}=$ $\left(\zeta_{R}^{14, \alpha} \pm \zeta_{R}^{15, \alpha}\right) / \sqrt{2}$ with equal and opposite mass eigenvalues of magnitude $\simeq \Lambda_{T C}$. We label these as $t_{R}^{1 j, \alpha}$. Let us consider the particle state created by the action of the adjoint of the operator

$$
\frac{1}{\sqrt{2}} \epsilon_{123 j k} \epsilon_{\alpha \beta} t_{R}^{1 j, \alpha}{ }^{T} C t_{R}^{1 k, \beta}
$$

on the vacuum. (As before, the $\epsilon_{\alpha \beta}$ is included not to represent an $\mathrm{SU}(2)_{H C}$-singlet contraction, since $\mathrm{SU}(2)_{H C}$ has been broken to $\mathrm{U}(1)_{H C}$ at the higher scale $\Lambda_{B H C}$; instead, it represents the antisymmetrization on $\alpha$ and $\beta$ produced automatically by the contraction with $\epsilon_{123 j k}$ and Fermi statistics.) This particle has a mass $\simeq 2 \Lambda_{T C} \simeq O(600) \mathrm{GeV}$, but, because of the extra binding energy due to the attractive $\mathrm{U}(1)_{H C}$ interaction, is lighter than the particle corresponding to (5.16). Note that the particle (5.18) is a technibaryon (and is bosonic, since $N_{T C}$ is even). It is expected to be lighter than other technibaryons and hence to be stable and can provide a dark matter candidate. Since this technibaryon is composed of SM-singlet fermions, its interactions with regular matter would be highly suppressed, since they would proceed only via exchange of ETC gauge bosons. The interactions between two of the particles (5.18) would also only proceed via higher-order exchanges and, in particular for the case of $\mathrm{U}(1)_{H C}$, van der Waals-type interactions, since it is a technisinglet and $\mathrm{U}(1)_{H C}$-singlet. 


\section{E. Global Symmetries and (Pseudo)-Nambu-Goldstone Bosons}

The ETC model of this paper is based on the gauge group (3.4), and contains a total of six, $U(1)$ global symmetries, associated with the representations (4.1), (4.2), and (4.4) of the ETC group. (The Nambu-Goldstone bosons associated with global symmetries carried by $\zeta$ and $\omega$ fields will be weakly coupled to SM fields, and therefore we disregard them here.) One can label these $U(1)$ 's according to the representation they act on as:

$$
U(1)_{Q_{L}} \times U(1)_{u_{R}} \times U(1)_{d_{R}} \times U(1)_{L_{L}} \times U(1)_{e_{R}} \times U(1)_{\psi_{R}} .
$$

Four linear combinations are spontaneously broken by the TC condensates, and one by the ETC condensate at scale $\Lambda_{1}$, leaving one exact global symmetry (to be identified with baryon number conservation $\mathrm{U}(1)_{B}$, generated by the sum of the generators of $\mathrm{U}(1)_{Q_{L}}, \mathrm{U}(1)_{u_{R}}$, and $\mathrm{U}(1)_{d_{R}}$ ) and five Nambu-Goldstone bosons. Of these five, one linear combination acquires a mass via ETC instantons, which explicitly break the global symmetries of the theory, and another can be identified with the Nambu-Goldstone boson associated with spontaneous breaking of $U(1)_{Y}$, so that it gets eaten to become the longitudinal component of the $Z^{0}$ vector boson. We are left with three exactly massless Nambu-Goldstone bosons, each of which, being a composite of SM non-singlets, has potentially dangerous couplings to SM fields. A similar situation arises also in conventional TC theories, where it is known [55] that two such massless states are present. We will comment on the possible solutions to this phenomenological problem at the end of this subsection.

While baryon number is not spontaneously broken, instanton effects could in principle induce in the low-energy effective theory operators leading to phenomenologically unacceptable $\Delta B=1$ (proton decay) and $\Delta B=2$ (neutron-antineutron oscillations) transitions. It is easy to see that this is not the case: the triangle anomaly $\left[U(1)_{B}\right]\left[S U(5)_{E T C}\right]^{2}$ sums up to zero, and so $U(1)_{B}$, being free from $S U(5)_{E T C}$ anomalies, is an exact symmetry (we neglect in this discussion $S U(2)_{L}$ electroweak instanton effects, which are negligible in the zero temperature limit). By contrast, lepton number (defined as the sum of the generators of $\mathrm{U}(1)_{L}, \mathrm{U}(1)_{e_{R}}$, and $\mathrm{U}(1)_{\psi_{R}}$ ) is spontaneously broken at the scale $\Lambda_{1}$. But, due to the assignment of $\psi$ fields to the antisymmetric representation of $S U(5)_{E T C}$, this symmetry is anomalous, and hence is explicitly broken by instanton effects. In order to clarify this issue, it is useful to exhibit the operator encoding in the low energy theory the information about ETC instanton effects.

Following the analysis by 't Hooft [52], one characterizes instanton-induced violation of global symmetries by constructing appropriate product(s) $\mathcal{O}$ involving all of the fields that are nonsinglets under the ETC gauge group with a coefficient involving a dimensionless factor $A(\Lambda)$ and a factor of the form $1 / \Lambda^{d_{\mathcal{O}}-4}$, where $d_{\mathcal{O}}$ denotes the dimension of $\mathcal{O}$ and $\Lambda$ 
denotes the relevant ETC scale. The quantity $A(\Lambda)$ consists of a power of $\pi / g_{E T C}(\Lambda)$ and an exponential factor $A(\Lambda)=\exp \left(-8 \pi^{2} / g_{E T C}^{2}(\Lambda)\right)$. For a weak-coupling situation such as electroweak $\mathrm{SU}(2)_{L}$, there is severe suppression due to the exponential factor; however, for the strong-coupling ETC theory under consideration here the power of $\pi / g_{E T C}$ can produce an enhancement comparable to the suppression from the exponential, and there is significant uncertainty in this dimensionless factor. Accordingly, for our estimates we take the dimensionless factor to be of order unity. In the present case we need to generalize the 't Hooft analysis (i) from a simple nonabelian group to a group which is a direct product of nonabelian factors; (ii) from a theory with fermions transforming only according to the fundamental representation to a theory with fermions transforming according to several different representations (fundamental, conjugate fundamental, and antisymmetric secondrank tensor representations for $\mathrm{SU}(5)_{E T C}$ ); and (iii) from a theory with only one gauge symmetry-breaking scale to a theory with a sequence of different breaking scales.

At the scale $\Lambda_{1}$, hypercolor interactions are still weak, and we neglect them together with the associated $\mathrm{SU}(2)_{H C}$ instantons. Since SM gauge interactions are even weaker at this scale, we also consider the limit in which SM gauge interactions are turned off. The ETC Lagrangian at the $\mathrm{SU}(5)_{E T C}$ symmetric level for energies $E>\Lambda_{1}$ is then invariant under the global symmetry

$$
G_{\text {glob. }}=\mathrm{U}(9) \times \mathrm{U}(6) \times \mathrm{U}(1) .
$$

To see this, we note that in this limit, the Lagrangian is invariant under transformations among the nine components of $Q_{L}$ and $d_{L}^{c}$, each of which is a 5 representation of $\mathrm{SU}(5)_{E T C}$, and among the six components of $L_{L}, e_{L}^{c}$, and $u_{L}^{c}$, each of which is a $\overline{5}$ representation. The additional $\mathrm{U}(1)$ is for $\psi_{R}$.

We construct an operator encompassing effects of ETC instantons by starting in the $\mathrm{SU}(5)_{E T C}$-symmetric theory, combining $\mathrm{SU}(5)_{E T C}$ fermion representations in such a manner as to respect the global symmetries, and incorporating the physics of the breaking of this group via the coefficient of the operator, which involves the breaking scale, $\Lambda_{1}$. Just as in the $\mathrm{SU}(2)_{L}$ case [52], the existence of this breaking scale cuts off integrations over instanton size and makes it possible to work with a local instanton operator.

For energies $E<\Lambda_{1}$, we obtain the local operator given by

$$
\frac{A\left(\Lambda_{1}\right)}{\Lambda_{1}^{32}} \mathcal{O}
$$

where $A\left(\Lambda_{1}\right) \simeq O(1)$ and, in a compact notation,

$$
\mathcal{O}=\left[\Pi u_{L} d_{L} d_{L}^{c}\right]\left[n_{L} e_{L} e_{L}^{c} u_{L}^{c}\right]\left[\Pi \psi_{L}^{c}\right]\left[\Pi \zeta_{L}^{c}\right]
$$


In Eq. (5.22) the meaning of the notation is as follows: (i) [ $\left.\Pi u_{L} d_{L} d_{L}^{c}\right]$ is an antisymmetrized 9 -fold product of fermion fields that including all $N_{c}=3$ colors and all flavors so as to yield an invariant under global SU(9) (ii) $\left[\Pi n_{L} e_{L} e_{L}^{c} u_{L}^{c}\right]$ is an antisymmetrized 6-fold product including a product over all colors, so as to yield an invariant under global SU(6); (iii) $\left[\Pi \psi_{L}^{c}\right]$ denotes a product over three $\psi_{L}^{c}$ fields, reflecting the fact that the contribution to the anomaly of a $\mathrm{U}(1)$ number transformation, and hence to the effective instanton operator, of a fermion field transforming according to the anti-symmetric rank-2 representation of $\mathrm{SU}(5)$ is 3 times that of a fermion transforming according to the fundamental representation; (iv) $\left[\Pi \zeta_{\alpha, L}^{c}\right]$ denotes a similar cubic product of the $\zeta$ fields for each value of the $\mathrm{HC}$ index, antisymmetrized on this index so as to yield an $\mathrm{SU}(2)_{H C}$-invariant. There are thus $N_{F}=24$ fermion fields in the operator $\mathcal{O}$, so that $d_{\mathcal{O}}=36$, and the dimensional coefficient in Eq. (5.21) is $1 / \Lambda_{1}^{32}$. Since $\Lambda_{1}$ is much larger than the electroweak symmetry-breaking scale, we also require that $\mathcal{O}$ be invariant under $G_{S M}$.

As anticipated, the operator $\mathcal{O}$ is invariant under baryon number $U(1)_{B}$ and transforms as $|\Delta L|=2$. We have already observed and made central use of the fact that lepton number is broken spontaneously by the bilinear ETC condensate (4.6) at scale $\Lambda_{1}$. Now, one could imagine generating a similar bilinear operator by contracting 22 of the 24 fields in (5.22) (all but two of the $\psi$ fields). The attendant integrations would be cut off at a scale no larger than $\Lambda_{1}$, and therefore the coefficient of the bilinear would be no larger than $\Lambda_{1}$. This operator would necessarily be an $\mathrm{SU}(5)_{E T C}$-non-singlet, and would consistently align in the same direction as (4.6) (taken to be the $i=1$ direction).

Finally, we return to the issue of the three exactly massless, electrically neutral, NambuGoldstone bosons enumerated above. Since the operator $\mathcal{O}$ breaks $\mathrm{U}(1)_{\psi_{R}}$, each of these necessarily includes SM non-singlet constituents. In our model, where these particles are formed at the electroweak (technicolor) scale, this is phenomenologically unacceptable [54]. The model of this paper must therefore be regarded as incomplete. Some new, higher energy interactions must be invoked to break explicitly the three corresponding global symmetries. An example of such interactions is discussed in Ref. [55], in the context of ETC theories which employ vector-like representations for SM fermions: a four-fermion operator that one could obtain in a Pati-Salam extension of the SM gauge group is shown to give masses to massless states. It is not possible to construct a conventional Pati-Salam extension of the gauge symmetry of models in the class considered here, in which the left- and right-handed chiral components of the down-type quarks and charged leptons transform according to conjugate representations of the ETC group. However, one can envision a different type of extended gauge symmetry with interactions that would break these three global U(1) symmetries, if some of the ETC representations come from unified representations of a larger gauge 
symmetry group. Assuming such interactions to be present at scales not too far above $\Lambda_{1}$, and using Dashen's formula as in Ref. [55], masses of the order of $10 \mathrm{GeV}$ can be anticipated for these particles. They would then satisfy astrophysical and cosmological constraints [54], as well as constraints coming from the decay $\Upsilon(1 S) \rightarrow \gamma+$ pseudoscalar [32]. It will be important to refine these mass estimates in the context of an explicit extension of our model and to explore other phenomenological signatures of these particles.

We note that all other pseudo-Nambu-Goldstone bosons in our model acquire mass via QCD or ETC interactions, and are then heavy enough not to cause phenomenological problems. This is to be contrasted with ETC models which employ vector-like representations for the SM fermions. There, some (electrically charged, color neutral) pseudo-Nambu-Goldstone bosons acquire mass only through electroweak interactions, and thus remain relatively light. The assignment of up-type and down-type right-handed quarks to different ETC representations (and a similar assignment for the leptons) explicitly breaks (via ETC interactions) the symmetries giving rise to these additional light states.

\section{DISCUSSION AND CONCLUSIONS}

In this paper we have addressed the problem of quark and lepton masses and mixing angles in the ETC framework. The production of intra-generational mass splittings and CKM mixing has been a long-standing challenge for these theories. We have proposed a framework to achieve these goals and have illustrated it with a specific model. The model is based on the relation (2.1), which, with the choice $N_{T C}=2$, implies that the ETC group is $\mathrm{SU}(5)_{E T C}$. There are three reasons for using $N_{T C}=2$ : (i) it minimizes TC contributions to precision electroweak observables, in particular the $T$ and $S$ parameters, both of which are proportional to $N_{T C}$; (ii) with $N_{f} \simeq 8$ vectorially coupled technifermions (as is true of our model with both sequences 1 and 2), perturbative beta function calculations suggest that the technicolor theory will exhibit walking (approximately conformal) behavior which enhances both standard-model fermion masses and pseudo-Nambu-Goldstone boson masses; and (iii) the choice $N_{T C}=2$ allows our mechanism [6] to operate and lead to small but nonzero neutrino masses and intra-generational mass splittings.

We have formulated and analyzed an approach based on the assignment of the righthanded components of the $Q=-1 / 3$ quarks and charged leptons to a representation of the ETC gauge group that is conjugate to the representation of the corresponding left-handed components. This leads to a natural suppression of these masses relative to those of the $Q=$ $2 / 3$ quarks, as well as to nontrivial quark mixing angles. For the neutrinos, a set of (righthanded) standard-model singlets was included, and assigned to representations that also 
lead to a suppression of their masses, as well as to nontrivial mixing angles and a potential seesaw mechanism. We note that these assignments are ad hoc, made to accommodate the observed features of the fermion mass matrices. Within our framework, they do not offer a direct explanation of why the $Q=2 / 3$ quarks are heavier than the corresponding $Q=-1 / 3$ quarks in the higher two generations or why the colored fermions (quarks) are heavier than the corresponding color-singlet fermions (leptons). A natural goal for extensions of the present work would be to explain these correlations.

We have provided a simple illustrative model involving one additional strong gauge interaction denoted as hypercolor ( $\mathrm{HC})$, and an economical representation content. We identified two plausible sequences of ETC symmetry breaking in this model depending on the relative strengths of the ETC and HC interactions. Although this simple model is not fully realistic, it has many features of a successful theory.

With respect to the quarks and charged leptons, the model can generate a sufficiently heavy top quark and satisfactory inter-generational mass splittings in the up-quark sector. It naturally achieves the observed intra-generational mass splittings for the higher two generations: $m_{t}>>m_{b}, m_{\tau}$ and $m_{c}>>m_{s}$ and $m_{\mu}$; and some quark mixing. It achieves these splittings without prohibitively large contributions to $\Delta \rho=\alpha_{e m} T$. Of the two sequences considered, sequence 1 has three ETC breaking scales and can account for all the up-type quark masses. The down-type quark masses and charged lepton masses are suppressed, as desired, compared to these, because of the use of relatively conjugate ETC representations for the left- and right- handed components. But with the up-type quark masses fit, there is excessive suppression of down-quark and charged lepton masses, especially for those of the first generation. In sequence 2, there are only two ETC breaking scales. Hence, a hierarchy between $m_{c}$ and $m_{t}$ is more difficult to achieve, although the non-diagonal structure of $M^{(u)}$ offers a possibility, as we discussed. There is the same excessive suppression of firstgeneration down-quark and charged lepton masses. Although the model does not give fully realistic CKM mixing, it does offer the prospect of spontaneous (dynamical) generation of a $\mathrm{CP}$-violating phase, and this will be an interesting avenue for further study, in conjunction with implications for the strong CP problem. Leptonic CP violation in this class of models is also worthy of study.

For the neutrinos, sequence 1 has little success. It leads to Dirac masses suppressed relative to the $Q=2 / 3$ quark masses, to right-handed Majorana masses, and to some neutrino mixing. But the Dirac masses remain too large, and the Majorana masses are too small for seesaw mechanism. Sequence 2, which can occur if the HC coupling is somewhat weaker at the relevant scales, has advantages and disadvantages. A major advantage is that purely within the neutrino sector, it can incorporate the seesaw mechanism of Ref. [6], 
yielding experimentally acceptable light neutrino masses. However, the value of the lower ETC breaking scale $\Lambda_{23}$ that produces a successful fit to neutrino masses does not yield sufficiently large second or third-generation quark and lepton masses.

The are also some potential phenomenological problems. The class of models considered leads to flavor-changing neutral currents at a level that could exceed the measured values for $\Delta m_{K}$ and $\Delta m_{B_{d}}$, and to a value for $\Delta m_{B_{s}}$ well in excess of the current range of SM predictions. This could be ameliorated if the model exhibited walking beyond the lowest ETC scale. This class of models also has a potential problem with massless (electrically neutral) Nambu-Goldstone bosons. One can envision additional interactions that will raise their masses to the level of $10 \mathrm{GeV}$ or higher, satisfying current experimental bounds.

The shortcomings of the simple model and the symmetry breaking patterns considered here, suggest a variety of directions for further exploration. An evident problem is the limited number of gauge boson mixings which yield many zero entries in the quark and lepton mass matrices. These zeros are traceable to selection rules related to residual global symmetries. But since these selection rules seem specific to the breaking patterns considered, one can imagine that other breaking patterns, if they are favored dynamically, could lead to more nonzero mass entries and hence more realistic quark and lepton mixing.

One possibility along these lines can arise if the interaction strengths are such that the condensates of both sequences 1 and 2 form. This involves the occurrence of misaligned condensates, i.e., condensates occurring at comparable energy scales that respect different symmetries. This could improve the predictions of fermion mixing, but would have the drawback that there would be only two ETC scales, $\Lambda_{1}$ and $\Lambda_{23}$. This contrasts with sequence 1 , which has three such scales, in one-to-one correspondence with the generations, and sequence 2 , which has two ETC scales $\Lambda_{1}$ and $\Lambda_{23}$, but also an additional scale $\Lambda_{B H C}$ associated with the breaking of hypercolor. Consequently, while the misaligned-condensate possibility may produce more complete and realistic quark and lepton mixing, it seems unable to produce realistic scales for quark, lepton, or neutrino masses.

Another direction is to retain the group structure, (3.4), of the model, but to consider different fermion representations. An example is a model of DES type (see Section II.D for a definition) in which the SM-nonsinglet fermions transform according to (4.1) and

$$
L_{L}:(5,1,1,2)_{-1, L}, \quad e_{R}:(\overline{5}, 1,1,1)_{-2, R},
$$

and the SM-singlet fermions transform as

$$
\psi_{R}^{i j}:(10,1,1,1)_{0, R}, \quad \zeta_{R}^{i j, \alpha}:(10,2,1,1)_{0, R}, \quad A_{j k, R}^{i}:(\overline{45}, 1,1,1)_{0, R} .
$$

This model includes a mixed tensor representation of $\mathrm{SU}(5)_{E T C}$, the $(\overline{45}, 1,1,1)_{0, R}=A_{j k, R}^{i}$. The mixed tensor representation $A_{j k}^{i}$ is antisymmetric in the indices $j, k$ and satisfies the 
tracelessness condition $\sum_{i=1}^{N} A_{i k}^{i}=0$, which amounts to $N$ equations, so that its dimension is $N(N-2)(N+1) / 2$. The contributions of the SM-singlet fermions to the $\mathrm{SU}(5)_{E T C}$ gauge anomaly satisfy the DES subcase of condition (4.3): $A\left(\psi_{R}\right)=1, A\left(\zeta_{R}\right)=2, A(A)=$ $-A\left(45_{S U(5)}\right)=6$. It is therefore anomaly-free. Although the particular model of this type that we studied did not lead to an overall improvement in predictions, we believe that the analysis of models with different types of fermion representations is worthwhile.

Some of the defects of the class of models considered here, including the explicit model analyzed in section IV, derive from the fact that the Dirac submatrix that exerts dominant control over the generation of masses for the primary mass eigenstates in the observed electroweak-doublet neutrinos satisfies the relation (4.39). This, in turn, reflects the fact that there is only one, common mass suppression mechanism for the down-type quarks, charged leptons, and Dirac neutrino masses relative to the up-type quarks (the use of conjugate ETC representations for the left-and right-handed down-type quarks and charged leptons, and a similar arrangement for the left- and right-handed neutrinos.) The relation (4.39) prevents a simultaneous fit to all of these masses.

One salient property of the present class of models is that the $b_{i j}$ Dirac neutrino mass terms relevant to the seesaw connect the left-handed electroweak-doublet neutrinos to righthanded SM-singlet fermions whose masses arise via loop effects rather than directly, via participation in condensates. The models do contain a different type of Dirac mass term (the $d_{i, j k}$ ) which connect the left-handed electroweak-doublet neutrinos with right-handed SM-singlet fermions that participate directly in condensates and hence gain larger dynamical masses. In our models, the $d_{i, j k}$ terms do not play an important role in driving a seesaw mechanism. It would be valuable to develop models in which the $d_{i, j k}$ terms do play a central role, thus avoiding the constraint (4.39).

Two interesting directions for future research suggest themselves. First, it is important to construct extensions of the class of models considered here which contain interactions yielding sufficiently large masses for the light, SM-singlet Nambu-Goldstone bosons. Second, in the present work, and its progenitors $[22,6,8]$, the extended technicolor symmetry breaking at levels below $\Lambda_{1}$ involves a combination of the ETC interaction and another strongly coupled gauge interaction, namely hypercolor. It would be worthwhile to try to construct models in which all of the ETC symmetry breaking could be achieved without recourse to another gauge interaction that is strongly coupled at a similar energy scale. Ideally, one would be able to achieve both of these goals in a single new model.

This research was partially supported by the grants DE-FG02-92ER-4074 (T.A. and M.P.) and NSF-PHY-00-98527 (R.S.). We thank Sekhar Chivukula, Alex Kagan, and Ken Lane for helpful comments and acknowledge the Aspen Center for Physics, where some of 
this research was done. 


\section{APPENDIX A: ETC GAUGE BOSON MIXING}

The estimates in Section IV of the fermion mass matrices for our model depend critically on mixing among the ETC gauge bosons. Here we list for each symmetry-breaking sequence those mixings that are nonzero, and estimate their magnitudes. The fact that some mixings vanish can be understood from global symmetry considerations. This discussion is provided in Section IV for each symmetry-breaking sequence.

\section{Definitions and Identities}

For a fermion $f_{L}$, transforming, say, according to the fundamental representation of $\mathrm{SU}\left(N_{E T C}\right)$, the basic coupling is given by

$$
\mathcal{L}=g_{E T C} \bar{f}_{i, L} T_{a}\left(V_{a}^{\lambda}\right)_{j}^{i} \gamma_{\lambda} f_{L}^{j}
$$

where the $T_{a}$, with $1 \leq a \leq N_{E T C}^{2}-1$, are the generators of $\mathrm{SU}\left(N_{E T C}\right)$, and the $V_{a}$ are the corresponding ETC gauge bosons. Just as in weak interactions one switches from the Cartesian basis $A_{a}^{\lambda}$, with $a=1,2,3$, to a basis comprised of the linear combinations of gauge bosons $\left(W^{ \pm}\right)^{\lambda} \equiv\left(A_{1}^{\lambda} \mp i A_{2}^{\lambda}\right) / \sqrt{2}$ corresponding to the $\mathrm{SU}(2)$ shift operators $T_{ \pm}=T_{1} \pm i T_{2}$, so also it is convenient to use the analogous basis for the ETC gauge bosons corresponding to non-diagonal generators here. In the $\mathrm{SU}(2)$ case, one may label $W^{+}=V_{2}^{1}$ and $W^{-}=$ $V_{1}^{2}=\left(V_{2}^{1}\right)^{\dagger}$ (suppressing the Lorentz index), meaning that for a fermion in the fundamental representation, say $f_{L}=\left(\begin{array}{l}f^{1} \\ f^{2}\end{array}\right)$, one has a vertex in which $f_{L}^{1}$ absorbs a $V_{1}^{2}$ or emits a $V_{2}^{1}$, making a transition to $f_{L}^{2}$ with coupling $g_{w k} / \sqrt{2}$. Similarly, for $\mathrm{SU}\left(N_{E T C}\right)$ we use a basis in which the gauge bosons corresponding to non-diagonal, shift operators are labelled as $V_{j}^{i}=\left(V_{i}^{j}\right)^{\dagger}$, where $1 \leq i, j \leq N_{E T C}, i \neq j$. The associated vertex is $\left(g_{E T C} / \sqrt{2}\right) \bar{f}_{i, L}\left(V_{j}^{i}\right)^{\lambda} \gamma_{\lambda} f_{L}^{j}$. Analogous comments apply to the couplings of the ETC gauge bosons to the right-handed components of $f$, and also, with appropriate changes to theories with the left- or right-handed component of $f$ transforms according to a conjugate fundamental representation. We retain the Cartesian basis for the ETC gauge bosons corresponding to diagonal Cartan generators of $\mathrm{SU}\left(N_{E T C}\right)$ and hence write them as $V_{a}$, where $a=n^{2}-1$ for $2 \leq n \leq N_{E T C}$.

To discuss the ETC gauge boson mixings that will be important for generating various contributions to fermion mass matrices, we consider the one-particle-irreducible vacuum polarization tensor ${ }_{n}^{k} \Pi_{j}^{i}(q)_{\mu \lambda}$ producing the transition $V_{j}^{i} \rightarrow V_{n}^{k}$. We assume that the diagonal masses of these fields have already been included, and therefore exclude the case $i=k, j=n$ from consideration here. Because the momenta in the loop in Fig. 2 and its analogue for the charged leptons are cut off by the technicolor dynamical mass insertion on the internal 
technifermion line at a scale $\Lambda_{T C}$ which is small compared to the lowest ETC scale, we shall need only the expressions for ETC gauge boson mixing evaluated effectively at zero momentum, i.e., ${ }_{n}^{k} \Pi_{j}^{i}(0)_{\mu \lambda}$. While ${ }_{n}^{k} \Pi_{j}^{i}(q)_{\mu \lambda}$ must be transverse because the underlying currents are conserved, the appearance of a Nambu-Goldstone pole associated with the symmetry breaking means that ${ }_{n}^{k} \Pi_{j}^{i}(0)_{\mu \lambda}$ can be nonzero. We focus on the $g_{\mu \nu}$ piece (it is simplest to imagine working in Landau gauge for this purpose) and define the zero-momentum coefficient of $g_{\mu \nu}$ to be ${ }_{n}^{k} \Pi_{j}^{i}(0)$.

Mixing among group eigenstates $\left(V_{j}^{i}\right)_{\mu}$, is in general to be expected, setting in as the $\mathrm{SU}(5)_{E T C}$ breaks sequentially to $\mathrm{SU}(2)_{T C}$. Because of the mixings among the ETC gauge bosons $V_{j}^{i}$, the corresponding mass eigenstates are comprised of linear combinations of these group eigenstates. Since these masses are hierarchical, it will be useful to continue to refer loosely to the "masses" of various ETC gauge bosons. When a gauge group $\mathrm{SU}(N)$ breaks to $\mathrm{SU}(N-1)$, the $2 N-1$ gauge bosons in the coset $\mathrm{SU}(N) / \mathrm{SU}(N-1)$ pick up mass. For example, as the energy decreases through the scale $\Lambda_{1}, \mathrm{SU}(5)_{E T C}$ breaks to $\mathrm{SU}(4)_{E T C}$ and nine ETC gauge bosons gain masses. These consist of the four gauge bosons $\left(V_{1}^{i}\right)_{\mu}$, $i=2,3,4,5$, together with their adjoints $\left(V_{i}^{1}\right)_{\mu}$, and the the gauge boson corresponding to one diagonal generator. Similar comments apply for subsequent breakings. The existence of

mixing among these group eigenstates will be seen to occur in each of the symmetry breaking sequences considered here. In the presence of this mixing among ETC gauge bosons, one must rediagonalize the associated 2-point functions to obtain the actual mass eigenstates. Our procedure is equivalent to this diagonalization, to the order that we work. We note here that the relation $\left(V_{j}^{i}\right)_{\mu}^{\dagger}=\left(V_{i}^{j}\right)_{\mu}$, leads to some basic identities for ${ }_{n}^{k} \Pi_{j}^{i}(q)_{\mu \lambda}$ :

$$
\left|{ }_{n}^{k} \Pi_{j}^{i}(q)_{\mu \lambda}\right|=\left|{ }_{j}^{i} \Pi_{n}^{k}(q)_{\mu \lambda}\right|=\left|{ }_{i}^{j} \Pi_{k}^{n}(q)_{\mu \lambda}\right| .
$$

\section{ETC Gauge Boson Mixing for Symmetry-Breaking Sequence 1}

For the symmetry-breaking sequence 1, it turns out that the following ETC gauge boson mixings occur:

$$
\begin{gathered}
V_{1}^{4} \leftrightarrow V_{5}^{3}, \quad V_{1}^{5} \leftrightarrow V_{4}^{3}+(4 \leftrightarrow 5) \\
V_{2}^{4} \leftrightarrow V_{5}^{2}, \quad V_{2}^{5} \leftrightarrow V_{4}^{2} \\
V_{1}^{t} \leftrightarrow V_{3}^{t}, \quad t=4,5
\end{gathered}
$$


It is useful to classify these mixings according to their selection rules under the maximal subgroup $\mathrm{U}(3) \times \mathrm{SU}(2)_{T C}$ of $\mathrm{SU}(5)_{E T C}$, where the $\mathrm{U}(3)$ is the group of transformations operating on the three ETC generation indices $i=1,2,3$, while the $\mathrm{SU}(2)_{T C}$ subgroup operates on the remaining $i=4,5$ indices. Thus, the mixings of Eq. (A3) and (A4) are of the form

$$
(\overline{3}, 2) \leftrightarrow(3, \overline{2}) \approx(3,2)
$$

where the second equality $\overline{2} \approx 2$ follows from the fact that $\mathrm{SU}(2)$ has only (pseudo)real representations. The mixings of Eq. (A5) are of the form

$$
(3,2) \leftrightarrow(3,2)
$$

The mixings (A3) and (A4) were present in the $G_{a}$ sequence of Ref. [6]. Diagrams for the mixings (A3)-(A5) are given in Figs. 8, 9, and 10. The fact that only these mixings are nonzero is explained from global symmetry considerations in Section IV. One can see how the transformation property (A6) arises by inspection of the gauge-fermion vertices and dynamical mass insertions. Consider, for example, the graph in Fig. A3). The incoming $V_{1}^{4}$ transforms as $(\overline{3}, 2)$ under the maximal subgroup $\mathrm{U}(3)_{\text {gen. }} \times \mathrm{SU}(2)_{T C}$ of $\mathrm{SU}(5)_{E T C}$; at the leftmost vertex, it produces a virtual $\zeta \bar{\zeta}$ pair; here the $\bar{\zeta}$ is written as the $\zeta_{R}^{12, \alpha}$ moving backward on the loop and transforming as $(\overline{3}, 2,2)$ under $\mathrm{U}(3)_{\text {gen }} \times \mathrm{SU}(2)_{T C} \times \mathrm{SU}(2)_{H C}$, while the $\zeta$ in the pair is the $\zeta_{R}^{24, \alpha}$ moving forward on the loop and transforming as a $(3,2,2)$ under the above group. Thus, the $\mathrm{U}(3)_{\text {gen }}$. flow at the leftmost vertex involves three $\overline{3}$ 's flowing into the vertex, for a $\mathrm{U}(3)_{\text {gen. }}$ singlet. The dynamical mass insertion on the upper fermion line of the loop, in which the $\zeta_{R}^{24, \alpha}$ goes to a $\zeta_{25, \beta, L}^{c}$ is such that two 2's of SU(2) $T C$ flow into the vertex, and two 2's of $\mathrm{SU}(2)_{H C}$ flow into the vertex, so that it is invariant under $\mathrm{SU}(2)_{T C}$ and $\mathrm{SU}(2)_{H C}$; however, this vertex transforms a $\overline{3}$ of $\mathrm{U}(3)_{\text {gen }}$. into a 3 of $\mathrm{U}(3)_{\text {gen. }}$. On the lower fermion line in the loop, reading in the direction of the arrow, from right to left, the $\zeta_{23, \beta, L}^{c}$ is transformed into a $\zeta_{R}^{12, \alpha}$, i.e., a 3 into a $\overline{3}$ of $\mathrm{U}(3)_{\text {gen. }}$. Combining these with the rightmost gauge-fermion vertex then yields the overall transformation property (A6) of this ETC gauge boson mixing. Similar analyses can be made for each of the other such diagrams.

Each nonzero mixing will be estimated from the corresponding graph(s) by assigning a dynamical mass to the fermions in each loop. Given the uncertainties associated with strong coupling, this can at most provide an order of magnitude estimate of the mixing. As an illustration, consider the mixing ${ }_{5}^{3} \Pi_{1}^{4}(0)$ arising from the graph in Fig. 8. (Recall that we will need this quantity only at zero momentum.) We estimate its size in terms of the integral (after Wick rotation) 


$$
\begin{aligned}
\left|{ }_{5}^{3} \Pi_{1}^{4}(0)\right| & =\left|{ }_{4}^{3} \Pi_{1}^{5}(0)\right| \simeq \frac{1}{2}\left(\frac{g_{E T C}}{\sqrt{2}}\right)^{2} \frac{N_{H C}}{4 \pi^{2}} \int \frac{\left(k^{2} d k^{2}\right) k^{4} \Sigma_{3}(k)^{2}}{\left(k^{2}+\Sigma_{3}^{2}\right)^{4}} \\
& \simeq \frac{g_{E T C}^{2} \Lambda_{3}^{2}}{24} .
\end{aligned}
$$

Here, the first factor of $1 / 2$ reflects the chiral nature of the gauge-fermion couplings, $N_{H C}=2$ arises from the sum over hypercolors in the loop, the $\left(k^{2} d k^{2}\right)$ is the measure after angular integration, the factor of $k^{4}$ arises from the numerators of the four fermion propagators, and $\Sigma_{i}$ is the dynamical mass of the fermions that condense at the scale $\Lambda_{i}$ (an ETC or TC scale).

The crude estimate, $g_{E T C}^{2} \Lambda_{3}^{2} / 24$, is made as follows. If the condensation occurs in an $\mathrm{SU}(N)$ gauge theory, then for Euclidean $k \lesssim \Lambda_{i}, \Sigma_{i}(k) \simeq \Sigma_{i, 0}=2 \pi f_{n} / \sqrt{N}$, where $f_{n}$ is the associated pseudoscalar decay constant. For example, in QCD, one can define the dynamical (constituent) quark mass as $m_{\rho} / 2$ or $m_{p, n} / N_{c}$; these definitions yield the values $385 \mathrm{MeV}$ and $313 \mathrm{MeV}$, respectively, which also indicate the size of the theoretical uncertainty in this quantity. Taking the average, $350 \mathrm{MeV}$, and comparing this with the value of $337 \mathrm{MeV}$ obtained from the above relation, one finds agreement to about $5 \%$. In general, using the relation $f_{i} \simeq\left(\Lambda_{i} / 2\right) \sqrt{3 / N}$ yields the relation $\Sigma_{i, 0} \simeq(\pi / \sqrt{3}) \Lambda_{i}$. This, too, works well in the case of QCD, where, with $\Lambda_{Q C D} \simeq 180 \mathrm{MeV}$, the right-hand side is $\simeq 330 \mathrm{MeV}$, in agreement, to within the uncertainty, with the $350 \mathrm{MeV}$ estimate given above for the constituent quark mass. For high momentum $k>\Lambda_{i}, \Sigma_{i}(k)$ has a behavior that depends on the type of theory: like $\Sigma_{i, 0}^{3} / k^{2}$ up to logarithms for a QCD-like theory, and, in contrast, roughly like $\Sigma_{i, 0}^{2} / k$ for a walking theory. In Eq. (A8) the condensation scale for the fermions in the loop is $\Lambda_{3}$, whence the appearance of $\Sigma_{3}(k)$. We make the conservative assumption that the TC theory walks only up to the lowest ETC scale, $\Lambda_{3}$, and hence use $\Sigma_{3}(k) \simeq \Sigma_{3,0}^{3} / k^{2}$ for the large- $k$ behavior of this dynamical mass.

Consider next the loop diagram for the mixing $V_{1}^{t} \leftrightarrow V_{3}^{t}$ with $t=4,5$, shown in Fig. 10, and involving fermion masses that are all of order $\Lambda_{3}$. Using the same approach as above (and taking into account the contributions of both $\omega_{\beta, p, R}^{c}$ fields with $p=1,2$ ), this yields

$$
\left|{ }_{1}^{t} \Pi_{3}^{t}(0)\right| \simeq \frac{g_{E T C}^{2} \Lambda_{3}^{2}}{12} \text { for } t=4,5 .
$$

The loop diagram for the mixing $V_{2}^{4} \leftrightarrow V_{5}^{2}$ shown in Fig. 9, involves fermion masses with two different scales, $\Lambda_{2}$ for $\zeta_{R}^{34, \alpha}$ and $\zeta_{15, \beta, L}^{c}$ (as well as $\zeta_{R}^{14, \alpha}$ and $\zeta_{35, \beta, L}^{c}$ ), and $\Lambda_{3}$ for $\zeta_{R}^{23, \alpha}$ and $\zeta_{12, \beta, L}^{c}$. In this case, the mass mixing contribution for each of the two set of $\zeta$ 's that contribute, summed over hypercolor, is

$$
\frac{1}{2}\left(\frac{g_{E T C}}{\sqrt{2}}\right)^{2} \frac{N_{H C}}{4 \pi^{2}} \int \frac{\left(k^{2} d k^{2}\right) k^{4} \Sigma_{2}(k) \Sigma_{3}(k)}{\left(k^{2}+\Sigma_{2}^{2}\right)^{2}\left(k^{2}+\Sigma_{3}^{2}\right)^{2}}
$$




$$
\begin{aligned}
& \simeq\left(\frac{g_{E T C}^{2} N_{H C}}{4 \pi^{2}}\right)\left(\frac{\Sigma_{3,0}^{3} \Sigma_{2,0}}{\Sigma_{2,0}^{2}}\right) \\
& \simeq \frac{g_{E T C}^{2}}{12}\left(\frac{\Lambda_{3}}{\Lambda_{2}}\right) \Lambda_{3}^{2} .
\end{aligned}
$$

Here since the loop momentum $k$ extends above the smaller condensation scale $\Lambda_{3}$, we have used the form $\Sigma_{3}(k) \simeq \Sigma_{3,0}^{3} / k^{2}$, and since the important contributions to the integral do not extend much beyond the higher scale $\Lambda_{2}$, we have used $\Sigma_{2}(k) \simeq \Sigma_{2,0}$. The $\Sigma_{2,0}^{2}$ in the denominator is from a propagator factor that can be pulled out of the integral, given its degree of convergence. Thus, more generally, a loop integral of this type has a quadratic dependence on the smaller of the two condensation scales, further suppressed by the ratio of the smaller to the larger scale. Multiplying by another factor of 2 to take account of the fact that, for each set of hypercolors, there are two sets of $\zeta$ 's contributing to the loop, we obtain the estimate

$$
\left|{ }_{5}^{2} \Pi_{2}^{4}(0)\right|=\left|{ }_{4}^{2} \Pi_{2}^{5}(0)\right| \simeq \frac{g_{E T C}^{2} \Lambda_{3}^{3}}{12 \Lambda_{2}} .
$$

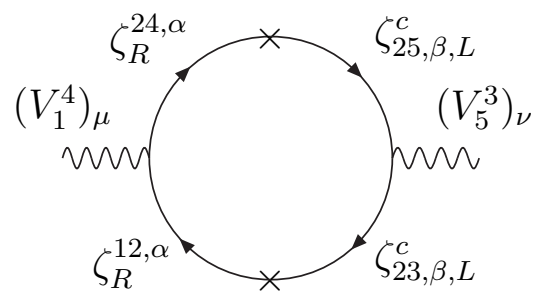

FIG. 8. A one-loop graph contributing to the ETC gauge boson mixing $V_{1}^{4} \leftrightarrow V_{5}^{3}$ in symmetry-breaking sequence 1. 


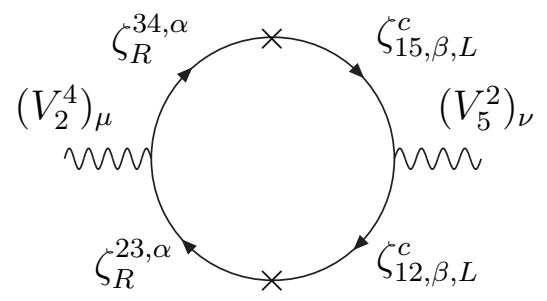

FIG. 9. A one-loop graph contributing to the ETC gauge boson mixing $V_{2}^{4} \leftrightarrow V_{5}^{2}$ in symmetry-breaking sequence 1. The analogous graph with the ETC indices 1 and 3 interchanged on internal fermion lines also contributes to this mixing.

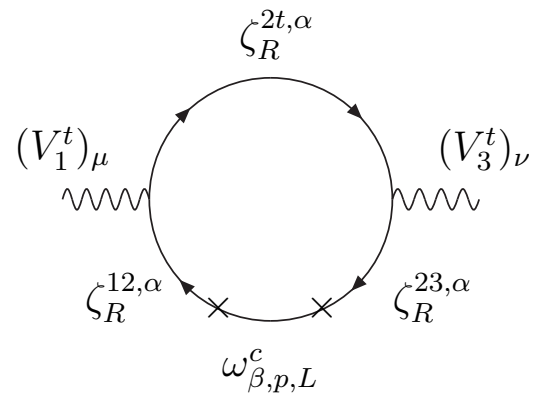

FIG. 10. A one-loop graph contributing to the ETC gauge boson mixing $V_{1}^{t} \leftrightarrow V_{3}^{t}$, where $t=4,5$, in symmetry-breaking sequence 1 .

Insertions of these non-diagonal mixings on ETC gauge boson lines give rise to further mixings. Thus,

$$
V_{1}^{4} \leftrightarrow V_{5}^{3} \leftrightarrow V_{5}^{1} \Longrightarrow V_{1}^{4} \leftrightarrow V_{5}^{1}
$$

and 


$$
V_{3}^{4} \leftrightarrow V_{5}^{1} \leftrightarrow V_{5}^{3} \Longrightarrow V_{3}^{4} \leftrightarrow V_{5}^{3}
$$

so that all of the three mixings,

$$
V_{i}^{4} \leftrightarrow V_{5}^{i}, \quad V_{i}^{5} \leftrightarrow V_{4}^{i}, \quad i=1,2,3
$$

are present with this sequence. We estimate

$$
{ }_{1}^{5} \Pi_{4}^{1}(0)={ }_{5}^{1} \Pi_{5}^{3}(0) \frac{1}{M_{3}^{2}}{ }_{5}^{3} \Pi_{1}^{4}(0) \simeq\left(\frac{g_{E T C}^{2} \Lambda_{3}^{2}}{12}\right) \frac{16}{\left(a g_{E T C} \Lambda_{3}\right)^{2}}\left(\frac{g_{E T C}^{2} \Lambda_{3}^{2}}{24}\right) \simeq \frac{g_{E T C}^{2} \Lambda_{3}^{2}}{18 a^{2}}
$$

and

$$
{ }_{5}^{3} \Pi_{3}^{4}(0)={ }_{5}^{3} \Pi_{5}^{1}(0) \frac{1}{M_{1}^{2}}{ }_{5}^{1} \Pi_{3}^{4}(0) \simeq\left(\frac{g_{E T C}^{2} \Lambda_{3}^{2}}{24}\right) \frac{16}{\left(a g_{E T C} \Lambda_{1}\right)^{2}}\left(\frac{g_{E T C}^{2} \Lambda_{3}^{2}}{12}\right) \simeq \frac{g_{E T C}^{2} \Lambda_{3}^{4}}{18 a^{2} \Lambda_{1}^{2}} .
$$

For the neutrino masses and mixing angles, we need an estimate of $r_{23}$ in the mass matrix $\left(M_{R}\right)_{\alpha \alpha}$ of Eq. (4.19), which plays the role as the large mass in the seesaw mechanism. This is generated as in Fig. 4. Since the dynamical mass insertion on the internal fermion line in this graph is of order the largest ETC scale, $\Lambda_{1}$, the loop momenta extend up to this scale, so that one needs to evaluate the ETC gauge boson mixing $V_{4}^{1} \leftrightarrow V_{1}^{5}$ (which occurs via the combination (A12)) for momenta $q$ up to $\Lambda_{1}$. Thus, in the contribution to this transition involving two successive fermion loops on the ETC gauge boson line, and hence four $\Lambda_{3}$-scale dynamical fermion mass insertions, each of these will be of the form $\Sigma_{3} \propto \Lambda_{3}^{3} / Q^{2}$, where $Q$ denotes the maximum of the incoming ETC gauge boson momentum $q$ and the fermion loop momentum $k$. This leads to a strong suppression of the resultant $r_{23}$.

ETC gauge boson loop diagrams with various insertions yield still further mixings. An example is shown in Fig. 11, which involves $V_{2}^{4} \rightarrow V_{5}^{2}$ and $V_{4}^{1} \rightarrow V_{4}^{3} \rightarrow V_{1}^{5}$ transition on virtual ETC gauge boson lines in the loop, yielding the overall transition $V_{2}^{1} \rightarrow V_{1}^{2}$.

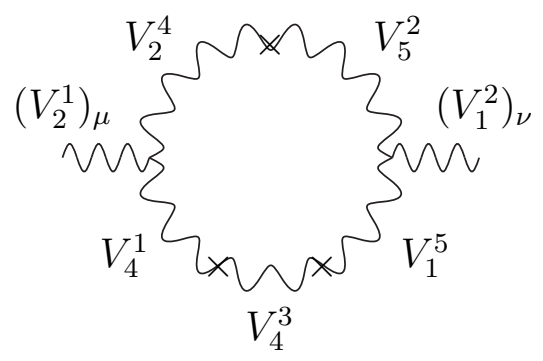


FIG. 11. One-loop graph contributing to the ETC gauge boson mixing $V_{2}^{1} \leftrightarrow V_{1}^{2}$ in sequence 1 . The indices on the ETC gauge bosons in the loop are written with the convention that both upper and lower lines go from left to right. The graph with indices 4 and 5 interchanged on the internal gauge boson lines also contributes.

The ETC gauge boson mixing $V_{2}^{3} \rightarrow V_{1}^{2}$ is generated, e.g., by a diagram analogous to Fig. 11, in which the upper and lower lines (reading from left to right) contain the respective transitions $V_{2}^{4} \rightarrow V_{5}^{2}$ and $V_{4}^{3} \rightarrow V_{4}^{1} \rightarrow V_{5}^{3} \rightarrow V_{1}^{5}$. The mixing $V_{3}^{1} \rightarrow V_{1}^{3}$ is generated, e.g., by an analogous diagram in which the upper and lower lines contain the respective transitions $V_{3}^{4} \rightarrow V_{1}^{4} \rightarrow V_{5}^{3}$ and $V_{4}^{1} \rightarrow V_{4}^{3} \rightarrow V_{1}^{5}$. The combination $V_{2}^{3} \leftrightarrow V_{1}^{2} \leftrightarrow V_{2}^{1} \leftrightarrow V_{3}^{2}$ yields the mixing $V_{2}^{3} \leftrightarrow V_{3}^{2}$.

From these mixings and the identity (A2), it follows that, for this model with any symmetry-breaking sequence,

$$
\left|M_{i j}^{(f)}\right|=\left|M_{j i}^{(f)}\right|
$$

\section{ETC Gauge Boson Mixing for Symmetry-Breaking Sequence 2}

For symmetry-breaking sequence 2, we obtain, to begin with, the ETC gauge boson mixings

$$
\begin{gathered}
V_{2}^{4} \leftrightarrow V_{5}^{3}, \quad V_{2}^{5} \leftrightarrow V_{4}^{3} \\
V_{1}^{t} \leftrightarrow V_{3}^{t}, \quad t=4,5
\end{gathered}
$$

and

$$
V_{4}^{i} \leftrightarrow V_{i}^{5}, \quad V_{5}^{i} \leftrightarrow V_{i}^{4}, \quad i=1,2,3
$$

The mixings (A18) and (A19) were present in the $G_{b}$ sequence of Ref. [6]. We show in Figs. 12 - 14 some diagrams that give rise to the mixings (A18)- (A20).

It is useful to note a general relation connecting the sequences $G_{a}$ and $G_{b}$ of Ref. [6] insofar as they involve condensates of the $\zeta$ fields and resultant ETC gauge boson mixings, namely that these condensates and mixings in sequence $G_{b}$ are related to those for sequence $G_{a}$ by the interchange of the ETC indices 1 and 2 (holding other ETC indices fixed) with appropriate changes in the condensation scale. Thus, (i) the condensate (4.27) occurring at scale $\Lambda_{2}$ in sequence 1 goes over, under this interchange of indices, to the condensate (4.48) occurring at scale $\Lambda_{B H C}$ in sequence 2 ; (ii) the condensate (4.29) at scale $\Lambda_{3}$ in sequence 1 
goes over to the condensate (4.53) at $\Lambda_{T C}$ in sequence 2; (iii) the condensate (4.30) at $\Lambda_{3}$ in sequence 1 goes over to (4.50) at $\Lambda_{23}$ in sequence 2 ; and (iv) the condensate (4.31) at $\Lambda_{3}$ in sequence 1 goes to the same condensate, now at $\Lambda_{23}$, in sequence 2 , and the condensate (4.32) at $\Lambda_{3}$ in sequence 1 goes to the $i=3$ case of (4.51) at $\Lambda_{23}$ in sequence 2. We shall denote this interchange symmetry with corresponding changes in condensation scales as $S 12$.

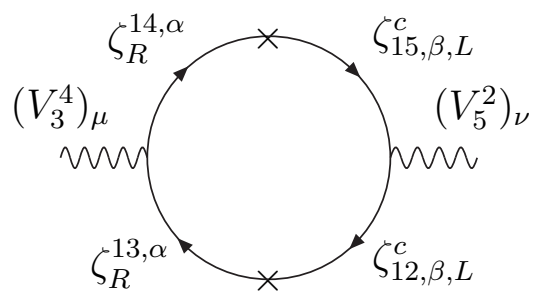

FIG. 12. A one-loop graph contributing to the ETC gauge boson mixing $V_{3}^{4} \leftrightarrow V_{5}^{2}$ for the symmetry-breaking sequence 2 .

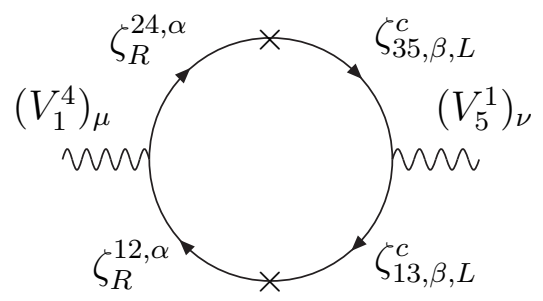

FIG. 13. A one-loop graph contributing to the ETC gauge boson mixing $V_{1}^{4} \leftrightarrow V_{5}^{1}$ for the symmetry-breaking sequence 2 . The graph with the index interchange $2 \leftrightarrow 3$ on the internal fermion lines also contributes. 


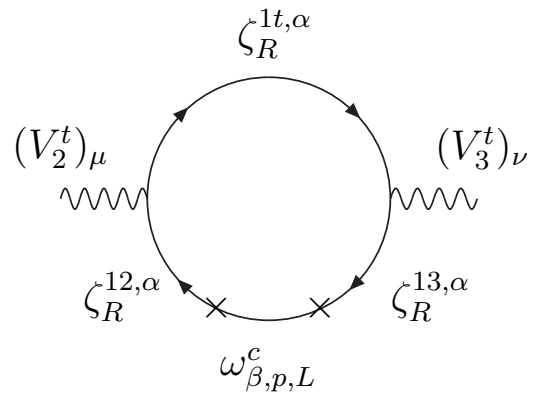

FIG. 14. A one-loop graph contributing to the ETC gauge boson mixing $V_{2}^{t} \leftrightarrow V_{3}^{t}$, where $t=4,5$, in symmetry-breaking sequence 2 .

By methods similar to those above, we estimate

$$
\begin{gathered}
\left|{ }_{5}^{3} \Pi_{2}^{4}(0)\right|=\left|{ }_{4}^{3} \Pi_{2}^{5}(0)\right| \simeq \frac{g_{E T C}^{2} \Lambda_{T C}^{3}}{24 \Lambda_{23}} \\
\left|{ }_{5}^{1} \Pi_{1}^{4}(0)\right|=\left|{ }_{4}^{1} \Pi_{1}^{5}(0)\right| \simeq \frac{g_{E T C}^{2} \Lambda_{23}^{3}}{12 \Lambda_{B H C}} \\
\left|{ }_{2}^{t} \Pi_{3}^{t}(0)\right| \simeq \frac{g_{E T C}^{2} \Lambda_{23}^{2}}{12} \text { for } t=4,5
\end{gathered}
$$

Note that for the mixing in Eq. (A22), for a given set of hypercolors, two pairs of $\zeta$ 's contribute, while in the case of (A23) we sum over $p=1,2$ for the contributions of the $\omega_{\beta, p, L}^{c}$

As was the case for sequence 1, insertions of these non-diagonal mixings on ETC gauge boson lines give rise to further mixings. Thus,

$$
V_{2}^{4} \leftrightarrow V_{5}^{3} \leftrightarrow V_{5}^{2} \Longrightarrow V_{2}^{4} \leftrightarrow V_{5}^{2}
$$

and

$$
V_{3}^{4} \leftrightarrow V_{5}^{2} \leftrightarrow V_{5}^{3} \Longrightarrow V_{3}^{4} \leftrightarrow V_{5}^{3}
$$

so that all of the three mixings 


$$
V_{i}^{4} \leftrightarrow V_{5}^{i}, \quad V_{i}^{5} \leftrightarrow V_{4}^{i}, \quad i=1,2,3
$$

occur. We estimate

$$
\begin{aligned}
& { }_{2}^{5} \Pi_{4}^{2}(0)={ }_{5}^{2} \Pi_{5}^{3}(0) \frac{1}{M_{23}^{2}}{ }_{5}^{3} \Pi_{2}^{4}(0) \simeq \frac{g_{E T C}^{2} \Lambda_{T C}^{2}}{18 a^{2}} \\
& { }_{3}^{5} \Pi_{4}^{3}(0)={ }_{3}^{5} \Pi_{2}^{5}(0) \frac{1}{M_{23}^{2}}{ }_{2}^{5} \Pi_{4}^{3}(0) \simeq \frac{g_{E T C}^{2} \Lambda_{T C}^{2}}{18 a^{2}}
\end{aligned}
$$

where again we note that there is significant theoretical uncertainty in the coefficients because of the strong ETC coupling. ETC gauge boson loop diagrams with various insertions yield still further mixings in a manner similar to that discussed above.

We summarize the ETC gauge boson mixings for the two symmetry-breaking sequences that we consider in this paper and, for comparison, the two simpler ones denoted $G_{a}$ and $G_{b}$ in Ref. [6], in Table I. The symmetry S12 connecting sequences 1 and 2 is evident here.

TABLE I. Some ETC gauge boson mixings in the model with the two symmetry-breaking sequences, denoted S1 and S2. For comparison, results for the two analogous sequences $G_{a}$ and $G_{b}$, without the $\omega_{p, R}^{\alpha}$ fields, are also listed. For each entry, a "y" indicates that the mixing occurs, while a blank indicates that it is absent. For each mixing of the form $V_{i}^{4} \leftrightarrow V_{5}^{j}$, it is understood that one also include the corresponding

\begin{tabular}{|c|c|c|c|c|}
\hline ETC transition & $G_{a}$ & S1 & $G_{b}$ & $\mathrm{~S} 2$ \\
\hline \multicolumn{5}{|l|}{$V_{1}^{4} \leftrightarrow V_{5}^{2}$} \\
\hline$V_{1}^{4} \leftrightarrow V_{5}^{3}$ & $\mathrm{y}$ & $\mathrm{y}$ & & \\
\hline$V_{2}^{4} \leftrightarrow V_{5}^{3}$ & & & $\mathrm{y}$ & $\mathrm{y}$ \\
\hline$\overline{V_{1}^{4} \leftrightarrow V_{5}^{1}}$ & & & $\mathrm{y}$ & $\mathrm{y}$ \\
\hline$V_{2}^{4} \leftrightarrow V_{5}^{2}$ & $\mathrm{y}$ & $\mathrm{y}$ & & $\mathrm{y}$ \\
\hline$V_{3}^{4} \leftrightarrow V_{5}^{3}$ & & $\mathrm{y}$ & & $\mathrm{y}$ \\
\hline \multicolumn{5}{|l|}{$\bar{V}_{1}^{t} \leftrightarrow V_{2}^{t}$} \\
\hline$V_{1}^{t} \leftrightarrow V_{3}^{t}$ & & $\mathrm{y}$ & & \\
\hline$V_{2}^{t} \leftrightarrow V_{3}^{t}$ & & & & $\mathrm{y}$ \\
\hline \multicolumn{5}{|l|}{$\overline{V_{2}^{1} \leftrightarrow V_{a}}$} \\
\hline \multicolumn{5}{|l|}{$V_{3}^{1} \leftrightarrow V_{a}$} \\
\hline \multicolumn{5}{|l|}{$V_{3}^{2} \leftrightarrow V_{a}$} \\
\hline$V_{1}^{2} \leftrightarrow V_{2}^{1}$ & & & $\mathrm{y}$ & $\mathrm{y}$ \\
\hline$V_{1}^{3} \leftrightarrow V_{3}^{1}$ & & & & $\bar{y}$ \\
\hline$V_{2}^{3} \leftrightarrow V_{3}^{2}$ & & & & $\mathrm{y}$ \\
\hline$V_{1}^{2} \leftrightarrow V_{2}^{3}$ & $\mathrm{y}$ & $\mathrm{y}$ & & \\
\hline$V_{1}^{2} \leftrightarrow V_{3}^{1}$ & & & $\mathrm{y}$ & $\mathrm{y}$ \\
\hline
\end{tabular}
transition with the indices 4 and 5 interchanged. For mixings of the form $V_{i}^{t} \leftrightarrow V_{j}^{t}$ with $i, j \in\{1,2,3\}$, the index $t$ takes on the values $t=4,5$. 
TABLE II. Elements of fermion mass matrices for the two symmetry-breaking sequences, denoted S1 and S2. For comparison, results for the two analogous sequences $G_{a}$ and $G_{b}$, without the $\omega_{p, R}^{\alpha}$ fields, are also listed. For each entry, a "y" and blank indicate that the entry is nonzero and zero, respectively. The symmetries $M_{i j}^{(f)}=M_{j i}^{(f)}$ are implicit.

\begin{tabular}{|c|c|c|c|c|}
\hline matrix element & $G_{a}$ & $\mathrm{~S} 1$ & $G_{b}$ & $\mathrm{~S} 2$ \\
\hline$M_{11}^{(u)}$ & $\mathrm{y}$ & $\mathrm{y}$ & $\mathrm{y}$ & $\mathrm{y}$ \\
\hline \multicolumn{5}{|l|}{$M_{12}^{(u)}$} \\
\hline$M_{13}^{(u)}$ & $\mathrm{y}$ & $\mathrm{y}$ & & \\
\hline$M_{22}^{(u)}$ & $\mathrm{y}$ & $\mathrm{y}$ & $\mathrm{y}$ & $\mathrm{y}$ \\
\hline$M_{23}^{(u)}$ & & & $\mathrm{y}$ & $\mathrm{y}$ \\
\hline$M_{33}^{(u)}$ & $\mathrm{y}$ & $\mathrm{y}$ & $\mathrm{y}$ & $\mathrm{y}$ \\
\hline$M_{11}^{(d, e)}$ & & $\mathrm{y}$ & $\mathrm{y}$ & $\mathrm{y}$ \\
\hline \multicolumn{5}{|l|}{$M_{12}^{(d, e)}, b_{12}$} \\
\hline$M_{13}^{(d, e)}, b_{13}$ & $\mathrm{y}$ & $\mathrm{y}$ & & \\
\hline$M_{22}^{(d, e)}, b_{22}$ & $\mathrm{y}$ & $\mathrm{y}$ & & $\mathrm{y}$ \\
\hline$M_{23}^{(d, e)}, b_{23}$ & & & $\mathrm{y}$ & $\mathrm{y}$ \\
\hline$M_{33}^{(d, e)}, b_{33}$ & & $\mathrm{y}$ & & $\mathrm{y}$ \\
\hline \multicolumn{5}{|l|}{$r_{22}$} \\
\hline$r_{23}$ & & $\mathrm{y}$ & $\mathrm{y}$ & $\mathrm{y}$ \\
\hline$r_{33}$ & & & & \\
\hline
\end{tabular}


[1] S. Weinberg, Phys. Rev. D 19, 1277 (1979); L. Susskind, Phys. Rev. D 20, 2619 (1979).

[2] S. Dimopoulos and L. Susskind, Nucl. Phys. B155, 237 (1979); E. Eichten and K. Lane, Phys. Lett. B90, 125 (1980).

[3] P. Sikivie, M. Shifman, M. Voloshin, and V. Zakharov, Nucl. Phys. B173, 189 (1980).

[4] B. Holdom, Phys. Lett. B150, 301 (1985); K Yamawaki, M. Bando, and K. Matumoto, Phys. Rev. Lett. 56, 1335 (1986); T. Appelquist, D. Karabali, and L. C. R. Wijewardhana, Phys. Rev. Lett. 57, 957 (1986); T. Appelquist and L. C. R. Wijewardhana, Phys. Rev. D 35, 774 (1987); Phys. Rev. D 36, 568 (1987).

[5] Recent reviews of dynamical symmetry breaking are R.S. Chivukula, hep-ph/0011264; K. Lane, hep-ph/0202255; C. Hill and E. Simmons, hep-ph/0203079.

[6] T. Appelquist and R. Shrock, Phys. Lett. B548, 204 (2002).

[7] T. Appelquist and R. Shrock, "Neutrino Masses in Theories with Dynamical Breaking of Electroweak and Extended Gauge Symmetries", in Proceedings of SCGT02, the International Workshop on Strongly Coupled Gauge Theories, Nagoya, Japan (Dec. 2002).

[8] T. Appelquist and R. Shrock, Phys. Rev. Lett. 90, 201801 (2003).

[9] T. Appelquist and J. Terning, Phys. Lett. B315, 139 (1993); T. Appelquist, J. Terning, L.C.R. Wijewardhana, Phys. Rev. Lett. 77, 1214 (1996); ibid. 79, 2767 (1997).

[10] T. Appelquist and F. Sannino, Phys. Rev. D 59, 067702 (1999); ibid. 60, 116007 (1999).

[11] Although we require our model to yield small $S$, we note that electroweak fits to precision electroweak data are complicated by the $\mathrm{NuTeV}$ anomaly reported in G. P. Zeller et al., Phys. Rev. Lett. 88, 091802 (2002). The conclusion that fits using just the $S$ and $T$ parameters do not yield good values of $\chi^{2}$ per degree of freedom has been reported, e.g., in S. Davidson, S. Forte, P. Gambino, N. Rius, and A. Strumia, JHEP 0202:037 (2002); W. Loinaz, N. Okamura, T. Takeuchi, and L. C. R. Wijewardhana, Phys. Rev. D 67, 073012 (2003).

[12] For a vectorial $\mathrm{SU}(N)$ theory with $N_{f}$ fermions in the fundamental representation, an approximate infrared-stable fixed point of the renormalization group equations may occur for $N_{f}$ values in the range of interest here. Assuming that a two-loop beta function calculation may be used to obtain the value of this approximate IRFP and applying the criticality condition [13], one infers that the theory should exist in a confining phase with spontaneous chiral symmetry breaking if $N_{f}<N_{f, \text { cond. }}$, where $N_{f, \text { cond. }} \simeq(2 / 5) N\left(50 N^{2}-33\right) /\left(5 N^{2}-3\right)$ and in a nonabelian Coulomb phase if $N_{f, \text { cond. }}<N_{f}<11 N / 2$. For $N=2$ we estimate $N_{f, \text { cond. }} \simeq 8$, but there is much theoretical uncertainty in this value because of the strong-coupling nature of the problem. For a review of attempts at nonperturbative lattice studies of these properties, see R. Mawhinney, Nucl. Phys. Proc. Suppl. 83, 57 (2000).

[13] In the approximation of a single-gauge-boson exchange, the critical coupling for chiral fermions transforming according to the representations $R_{1}$ and $R_{2}$ to form a condensate transforming as $R_{\text {cond. }}$ in an $\mathrm{SU}(N)$ gauge theory is given by the condition $\frac{3 \alpha}{2 \pi} \Delta C_{2}=1$, where $\Delta C_{2}=$ $\left[C_{2}\left(R_{1}\right)+C_{2}\left(R_{2}\right)-C_{2}\left(R_{c}\right)\right]$, and $C_{2}(R)$ is the quadratic Casimir invariant. 
[14] Recall that the number of fermion doublets of $\mathrm{SU}(2)$ must be even to avoid a global anomaly.

[15] J. Gasser, H. Leutwyler, Phys. Rept. 87, 77 (1982); H. Leutwyler, in Nucl. Phys. Proc. Suppl. 94, 108 (2001).

[16] For recent lattice measurements of light quark masses, see, e.g., the following, and references therein: A. Ali Khan et al. Phys. Rev. Lett. 85, 4674 (2000); J. Garden, et al., Nucl. Phys. B571, 237 (2000); M. Gockeler et al., Phys. Rev. D 62, 054504 (2000); M. Wingate et al., Int. J. Mod. Phys. A16 S1B, 585 (2001); L. Giusti, C. Hoelbling, and C. Rebbi, Phys. Rev. D 64, 114508 (2001); S. Aoki et al., Phys. Rev. D 67, 034503 (2003); D. Becirevic, V. Lubicz, and C. Tarantino, Phys. Lett. B558, 69 (2003).

[17] This value of $m_{u}$ is the current quark mass, i.e. the mass measured at a scale well above $\Lambda_{Q C D}$. We use the value $\left(m_{u}+m_{d}\right) / 2=4.3 \mathrm{MeV}$ [16] in conjunction with the current algebra result $m_{d} / m_{u}=1.8[15]$ to obtain $m_{u}=3.1 \mathrm{MeV}$.

[18] T. Appelquist, T. Takeuchi, M. Einhorn, and L. C. R. Wijewardhana, Phys. Lett. B 220, 223 (1989); T. Takeuchi, Phys. Rev. D 40, 2697 (1989); T. Appelquist, J. Terning, and L. C. R. Wijewardhana, Phys. Rev. D 44, 871 (1991) and references therein. See also B. Holdom, Phys. Rev. Lett. 60, 1233 (1988).

[19] C. Hill, Phys. Lett. B345, 483 (1995); K. Lane and E. Eichten, Phys. Lett. B352, 382 (1995); K. Lane, Phys. Rev. D 54, 2204 (1996); Phys. Lett. B433, 96 (1998).

[20] It is plausible that the fact that this inequality is different for the first generation, where $m_{e}<m_{u}<m_{d}$, is due to the dominant effect of off-diagonal entries in the up- and down-quark mass matrices in determining the respective lightest up and down quark masses.

[21] N. Cabibbo, Phys. Rev. Lett. 10, 531 (1963); M. Kobayashi and M. Maskawa, Prog. Theor. Phys. 49, 652 (1973).

[22] T. Appelquist and J. Terning, Phys. Rev. D 50, 2116 (1994).

[23] Z. Maki, M. Nakagawa, and S. Sakata, Prog. Theor. Phys. 28 (1962) 870 (2×2 matrix); see Ref. [24] for $3 \times 3$ matrix.

[24] B. W. Lee, S. Pakvasa, R. Shrock, and H. Sugawara, Phys. Rev. Lett. 38 (1977) 937.

[25] S. Weinberg, Phys. Rev. D 13, 974 (1976); M. Peskin, Nucl. Phys. B175, 197 (1980); J. Preskill, ibid. 177, 21 (1981). The TC theory forms condensates $\langle\bar{F} F\rangle$, where $F=U^{a}, D^{a}, E, N$, but not, e.g., $\left\langle\bar{U}_{a} E\right\rangle,\left\langle\bar{D}_{a} E\right\rangle,\left\langle\bar{U}_{a} N\right\rangle,\left\langle\bar{D}_{a} N\right\rangle,\left\langle\bar{U}_{a} D^{a}\right\rangle,\langle\bar{E} N\rangle$, or, for $N_{T C}=2,\left\langle\epsilon_{i j} F_{\chi}^{i T} C F_{\chi}^{\prime j}\right\rangle$, $\chi=L, R$. The excluded condensates would incur an energy price due to gauge boson mass generation when the (weaker) gauge symmetries are broken.

[26] Y. Fukuda et al., Phys. Lett. B433 (1998) 9; Phys. Rev. Lett. 81 (1998) 1562; ibid., 82 (1999) 2644; Phys. Lett. B467 (1999) 185; Phys. Rev. Lett. 85 (2000) 3999 (SuperK) and data from Kamiokande, IMB, Soudan-2, and MACRO experiments. Published fits to this data explain it via $\nu_{\mu} \rightarrow \nu_{\tau}$ oscillations with $\left|\Delta m_{32}^{2}\right| \simeq 3 \times 10^{-3} \mathrm{eV}^{2}$ and a maximal value of the associated mixing angle factor $\sin ^{2} 2 \theta_{23}=1$. The most recent SuperK data analysis leads to a slightly smaller value $\left|\Delta m_{32}^{2}\right| \simeq 2 \times 10^{-3} \mathrm{eV}^{2}$, as reported by $\mathrm{K}$. Lesko at the European Physical Society Meeting, Aachen, July, 2003. (The sign of $\Delta m_{32}^{2}$ is not determined by this data and is not yet known.)

[27] M. Ahn et al., Phys. Rev. Lett. 90, 041801 (2003).

[28] S. Fukuda, et al. Phys. Rev. Lett. 86 (2001) 5651, 5656; Phys. Lett. B539 (2002) 179 (SuperK); 
Q. Ahmad et al., Phys. Rev. Lett. 87 (2001) 071301; ibid. 89 (2002) 011301, 011302 (SNO). Other data is from the Homestake, Kamiokande, GALLEX, and SAGE experiments. The optimal fit to this data involves $\nu_{e}$ oscillations into $\nu_{\mu}$ and $\nu_{\tau}$ with $\Delta m_{21}^{2} \simeq 0.7 \times 10^{-4} \mathrm{eV}^{2}$, where $\Delta m_{i j}^{2}=m\left(\nu_{i}\right)^{2}-m\left(\nu_{j}\right)^{2}$, and a relatively large associated mixing angle $\tan ^{2} \theta_{12} \simeq 0.4$.

[29] K. Eguchi et al., Phys. Rev. Lett. 90, 021802 (2003).

[30] In addition to $\theta_{23}$ and $\theta_{12}$, the $(3 \times 3$ truncation) of the lepton mixing matrix depends on a rotation angle $\theta_{13}$ and a CP-violating phase $\delta$ (together with two Majorana phases that cannot be measured in neutrino oscillation experiments). The CHOOZ experiment (M. Apollonio et al., Phys. Lett. B420, 397 (1998); ibid., B466, 415 (1999)) yields the bound $\sin ^{2} 2 \theta_{13} \lesssim 0.1$. Discussions of options for measurements of $\theta_{13}$ and $\delta$ include V. Barger et al., hep-ph/0103052; Y. Itow et al., hep-ex/0106019; D. Ayres et al., hep-ex/0210005; H. Minakata et al., hepph/0211111; P. Huber, M. Lindner, T. Schwetz, W. Winter, hep-ph/0303232; S. Choubey, S. Petcov, M. Piai, hep-ph/0306017.

[31] M. Peskin and T. Takeuchi, Phys. Rev. D 46, 381 (1992).

[32] See http://pdg.lbl.gov for current data and limits.

[33] R. S. Chivukula, S. Selipsky, and E. Simmons, Phys. Rev. Lett. 69, 575 (1992);R. S. Chivukula, E. Gates, E. Simmons, and J. Terning, Phys. Lett. B311, 157 (1993); G.-H. Wu, Phys. Rev. Lett. 74, 4137 (1995).

[34] For a recent review and references, see, e.g., F. Parodi, in Proceedings of the Fifth International Conference on Hyperons, Charm, and Beauty Hadrons, ed. C. Kalman et al., Nucl. Phys. B (Proc. Suppl.) 115, 212 (2003); http://cern.ch/ckm-workshop.

[35] For a recent review, see N. Yamada, in Lattice 2002 - Proceedings of the XXth International Symposium on Lattice Field Theory, eds. R. Edwards, J. Negele, and D. Richards, Nucl. Phys. B (Proc. Suppl.) 119, 93 (2003).

[36] A. J. Buras, S. Jager, and J. Urban, Nucl. Phys. B 605, 600 (2001).

[37] B. W. Lee and R. Shrock, Phys. Rev. D 16, 1444 (1977).

[38] R. Appel et al., Phys. Rev. Lett. 85, 2450 (2000). This experiment has analyzed additional data, which could decrease the upper limit on $B R\left(K^{+} \rightarrow \pi^{+} \mu^{+} e^{-}\right)$somewhat (private communication from M. Zeller, spokesman of BNL E865).

[39] D. Ambrose et al., Phys. Rev. Lett. 81, 5734 (1998).

[40] R. Shrock, Phys. Lett. B96, 159 (1980); Phys. Rev. D24, 1232 (1981).

[41] S. Adler et al., Phys. Rev. Lett. 79, 2204 (1997); Phys. Rev. Lett. 84, 3768 (2000); Phys. Rev. Lett. 88, 041803 (2002).

[42] Private communication from L. Littenberg, cospokesman of BNL E787.

[43] R. Shrock, Phys. Rev. D24, 1275 (1981); Phys. Lett. B112, 382 (1982).

[44] An alternate definition for $\left|\bar{\nu}_{e}\right\rangle$ is the antineutrino that is emitted in a nuclear beta decay of the form $(Z, A) \rightarrow(Z+1, A)+e^{-}+\bar{\nu}_{e}$, and similarly for $\left|\nu_{e}\right\rangle$ as the neutrino emitted in the decay $(Z, A) \rightarrow(Z-1, A)+e^{+}+\nu_{e}$ or the electron-capture process $(Z, A)+e^{-} \rightarrow(Z-1, A)+\nu_{e}$.

[45] Implications for measurements of weak interaction quantities such as $G_{F}$ and $B R\left(\pi^{+} \rightarrow\right.$ $\left.e^{+} \nu_{e}\right) / B R\left(\pi^{+} \rightarrow \mu^{+} \nu_{\mu}\right)$ decays are discussed in [37,40].

[46] For recent reviews, see, e.g., S. Elliott and P. Vogel, Ann. Rev. Nucl. Part. Sci. 52, 115 (2002); O. Cremonesi, in Proceedings of Neutrino-2002 (hep-ex/0210007); S. Elliott, in R. Shrock, ed., 
Proceedings of the YITP Conference on Neutrinos and Implications for Physics Beyond the Standard Model, (nucl-ex/0301011). (We also note that a positive signal has been claimed in H. Klapdor-Kleingrothaus et al., Mod. Phys. Lett. A 16, 2409 (2001); hep-ph/0201231.)

[47] L. Littenberg and R. Shrock, Phys. Rev. Lett. 68443 (1992); Phys. Rev. D46 (Rapid Commun.), R892 (1992); Phys. Lett. B491, 285 (2000).; C. Dib et al., Phys. Lett. B493, 82 (2000).

[48] J. Kneller, R. Scherrer, G. Steigman, T. Walker, Phys. Rev. D 64, 123506 (2001); K. Olive, G. Steigman, T. Walker, Phys. Rept. 333, 389 (2000); G. G. Raffelt, in http://pdg.lbl.gov, op. cit.

[49] For recent limits, see, e.g, CDMS Collab., hep-ex/0306001 and references therein.

[50] Parenthetically, we note the values of the exponential coupling-dependent factor. To obtain these we use the fact that at a given energy scale $\mu$ the ETC coupling $\alpha_{E T C}(\mu)=g_{E T C}^{2}(\mu) /(4 \pi)$ satisfies the approximate criticality relation [13] $3 \alpha_{E T C} \Delta C_{2} /(2 \pi) \simeq 1$. Solving the criticality relation for $\alpha_{E T C}(\mu)$ and substituting in the expression for $A(\mu)$, we obtain $A(\mu)=$ $\exp \left(-8 \pi^{2} / g_{E T C}^{2}(\mu)\right) \simeq \exp \left(-3 \Delta C_{2}\right)$ at each particular ETC scale. In sequence 1 we have $\Delta C_{2}=24 / 5$ for the condensation at $\Lambda_{1}$, whence $A\left(\Lambda_{1}\right) \simeq \exp (-72 / 5) \simeq 6 \times 10^{-7}$; by similar means we have $A\left(\Lambda_{2}\right) \simeq e^{-15 / 2} \simeq 6 \times 10^{-4}$ and $\left.A\left(\Lambda_{3}\right) \simeq e^{-4} \simeq 0.02\right)$. The power-law prefactor also contributes to the dimensionless coefficient. As indicated in the text, because of the strongcoupling nature of the ETC theory at the relevant scales, we will simply use an effective-field theory approach of approximating this dimensionless coefficient as roughly unity.

[51] Y. Chikashige, R. Mohapatra, and R. Peccei, Phys. Lett. B98, 265 (1981); G. Gelmini, S. Nussinov, and T. Yanagida, Nucl. Phys. B219, 31 (1983).

[52] G. 't Hooft, Phys. Rev. Lett. 37, 8 (1976); Phys. Rev. D 14, 3432 (1978).

[53] We recall the standard definition for the Casimir invariant $T(R)$ : for a representation $R$ of a group $\mathrm{SU}(N), \sum_{i, j=1}^{\operatorname{dim}(R)} D_{R}\left(T_{a}\right)_{j}^{i} D_{R}\left(T_{b}\right)_{i}^{j}=T(R) \delta_{a b}$, where $D_{R}\left(T_{a}\right)$ is the generator $T_{a}$ in this representation, with $a, b$ denoting group indices and $i, j$ denoting the representation indices.

[54] see G. G. Raffelt, in Ref. [48].

[55] T. Appelquist and L. C. R. Wijewardhana, Phys. Rev. D 36, 568 (1987). 